\title{
PRESERVATION OF SPLITTING FAMILIES AND CARDINAL CHARACTERISTICS OF THE CONTINUUM*
}

BY

\section{Martin Goldstern And JAKOB Kellner}

Institut für Diskrete Mathematik und Geometrie, TU Wien, 1040 Vienna, Austria e-mail: martin.goldstern@tuwien.ac.at, kellner@fsmat.at URL: http://www.tuwien.ac.at/goldstern/, http://dmg.tuwien.ac.at/kellner

AND

\section{Diego A. MEJÍA}

Creative Science Course (Mathematics), Faculty of Science

Shizuoka University, Ohya 836, Suruga-ku, Shizuoka-shi 422-8529, Japan

e-mail: diego.mejia@shizuoka.ac.jp

URL: http://www.researchgate.com/profile/Diego_Mejia2

AND

\section{SAHARON SHELAH}

Einstein Institute of Mathematics, The Hebrew University of Jerusalem

Givat Ram, Jerusalem 91904, Israel

and

Department of Mathematics, Rutgers University, New Brunswick, NJ 08854, USA e-mail:shlah@math.huji.ac.il

URL: http://shelah.logic.at

\footnotetext{
* This work was supported by the following grants: Austrian Science Fund (FWF): project number I3081, P29575 (first author); P30666 (second author); Grant-inAid for Early Career Scientists 18K13448, Japan Society for the Promotion of Science (third author); Israel Science Foundation (ISF) grant no: 1838/19 (fourth author). This is publication number 1199 of the fourth author.

(C) The authors 2018. This article is published with open access at link.springer.com.

Open Access This article is distributed under the terms of the Creative Commons Attribution 4.0 International License (https://creativecommons.org/licenses/by/4.0/), which permits unrestricted use, distribution and reproduction in any medium, provided the appropriate credit is given to the original authors and the source, and a link is provided to the Creative Commons license, indicating if changes were made.
}

Received July 282020 and in revised form October 19, 2020 


\section{ABSTRACT}

We show how to construct, via forcing, splitting families that are preserved by a certain type of finite support iterations. As an application, we construct a model where 15 classical characteristics of the continuum are pairwise different, concretely: the 10 (non-dependent) entries in Cichon's diagram, $\mathfrak{m}(2$-Knaster $), \mathfrak{p}, \mathfrak{h}$, the splitting number $\mathfrak{s}$ and the reaping number $\mathfrak{r}$.

\section{Introduction}

In this paper we present a method to preserve certain splitting families along finite support iterations. These splitting families are constructed via forcing, using specific uncountable 2-edge-labeled graphs ${ }^{1}$ as support. The main application of this method is a forcing model where many classical cardinal characteristics of the continuum are pairwise different, including the splitting number $\mathfrak{s}$ and the reaping number $\mathfrak{r}$.

We assume that the reader is familiar with Cichon's diagram (Figure 1) containing the characteristics that we will call Cichon-characteristics. We also investigate some of the characteristics in the Blass diagram [Bla10, p. 481]. Figure 2 illustrates both diagrams combined, along with all the ZFC-provable inequalities that we are aware of. See [Bla10, BJ95] for the definitions and the proofs for the inequalities (with the exception of $\operatorname{cof}(\mathcal{M}) \leq \mathfrak{i}$, which was proved in [BHHH04]). In the following, we only give the definitions of the non-Cichoncharacteristics that we will investigate in this paper.

Definition 1.1: (1) For $a, b \in[\omega]^{\aleph_{0}}$, we define $a \subseteq^{*} b$ iff $a \backslash b$ is finite;

(2) and we say that $a$ splits $b$ if both $a \cap b$ and $b \backslash a$ are infinite, that is, $a \nsupseteq^{*} b$ and $\omega \backslash a \nsupseteq^{*} b$.

(3) $F \subseteq[\omega]^{\aleph_{0}}$ is a splitting family if every $y \in[\omega]^{\aleph_{0}}$ is split by some $x \in F$. The splitting number $\mathfrak{s}$ is the smallest size of a splitting family.

(4) $D \subseteq[\omega]^{\aleph_{0}}$ is an unreaping family if no $x \in[\omega]^{\aleph_{0}}$ splits every member of $D$. The reaping number $\mathfrak{r}$ is the smallest size of an unreaping family.

(5) $D \subseteq[\omega]^{\aleph_{0}}$ is groupwise dense when:

(i) if $a \in[\omega]^{\aleph_{0}}, b \in D$ and $a \subseteq^{*} b$, then $a \in D$,

(ii) if $\left\langle I_{n}: n<\omega\right\rangle$ is an interval partition of $\omega$ then $\bigcup_{n \in a} I_{n} \in D$ for some $a \in[\omega]^{\aleph_{0}}$

\footnotetext{
1 A 2-edge-labeled graph is a simple graph whose edges are labeled by either 0 or 1 .
} 
The groupwise density number $\mathfrak{g}$ is the smallest size of a collection of groupwise dense sets whose intersection is empty.

(6) The distributivity number $\mathfrak{h}$ is the smallest size of a collection of dense subsets of $\left\langle[\omega]^{\aleph_{0}}, \subseteq^{*}\right\rangle$ whose intersection is empty.

(7) Say that $a \in[\omega]^{\aleph_{0}}$ is a pseudo-intersection of $F \subseteq[\omega]^{\aleph_{0}}$ if $a \subseteq{ }^{*} b$ for all $b \in F$.

(8) The pseudo-intersection number $\mathfrak{p}$ is the smallest size of a filter base of subsets of $[\omega]^{\aleph_{0}}$ without pseudo-intersection.

(9) The tower number $\mathfrak{t}$ is the smallest length of a (transfinite) $\subseteq{ }^{*}$-decreasing sequence in $[\omega]^{\aleph_{0}}$ without pseudo-intersection.

(10) Given a class $\mathcal{P}$ of forcing notions, $\mathfrak{m}(\mathcal{P})$ denotes the minimal cardinal $\kappa$ such that, for some $Q \in \mathcal{P}$, there is some collection $\mathcal{D}$ of size $\kappa$ of dense subsets of $Q$ without a filter in $Q$ intersecting every member of $\mathcal{D}$.

(11) Let $\mathbb{P}$ be a poset. A set $A \subseteq \mathbb{P}$ is $k$-linked (in $\mathbb{P}$ ) if every $k$-element subset of $A$ has a lower bound in $\mathbb{P} . A$ is centered if it is $k$-linked for all $k \in \omega$.

(12) A poset $\mathbb{P}$ is $k$-Knaster, if for each uncountable $A \subseteq \mathbb{P}$ there is a $k$-linked uncountable $B \subseteq A$. And $\mathbb{P}$ has precaliber $\aleph_{1}$, if such a $B$ can be chosen centered. For notational convenience, 1-Knaster means ccc, and $\omega$-Knaster means precaliber $\aleph_{1}$.

(13) For $1 \leq k \leq \omega$ denote $\mathfrak{m}_{k}:=\mathfrak{m}\left(k\right.$-Knaster) and $\mathfrak{m}:=\mathfrak{m}_{1}$. We also set $\mathfrak{m}_{0}:=\aleph_{1}$.

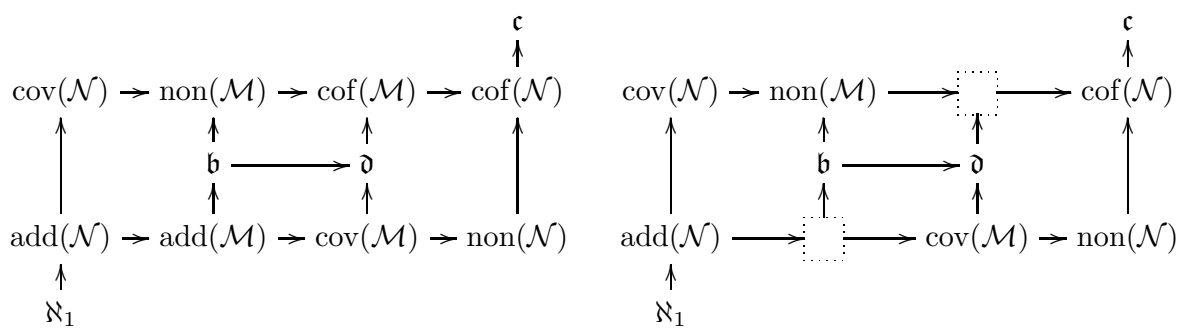

Figure 1. Cichon's diagram (left). In the version on the right, the two "dependent" values $\operatorname{add}(\mathcal{M})=\min \{\mathfrak{b}, \operatorname{cov}(\mathcal{M})\}$ and $\operatorname{cof}(\mathcal{M})=\max \{\operatorname{non}(\mathcal{M}), \mathfrak{d}\}$ are removed; the "independent" ones remain (nine entries excluding $\aleph_{1}$, or ten including it). An arrow $\mathfrak{x} \rightarrow \mathfrak{y}$ means that ZFC proves $\mathfrak{x} \leq \mathfrak{y}$. 


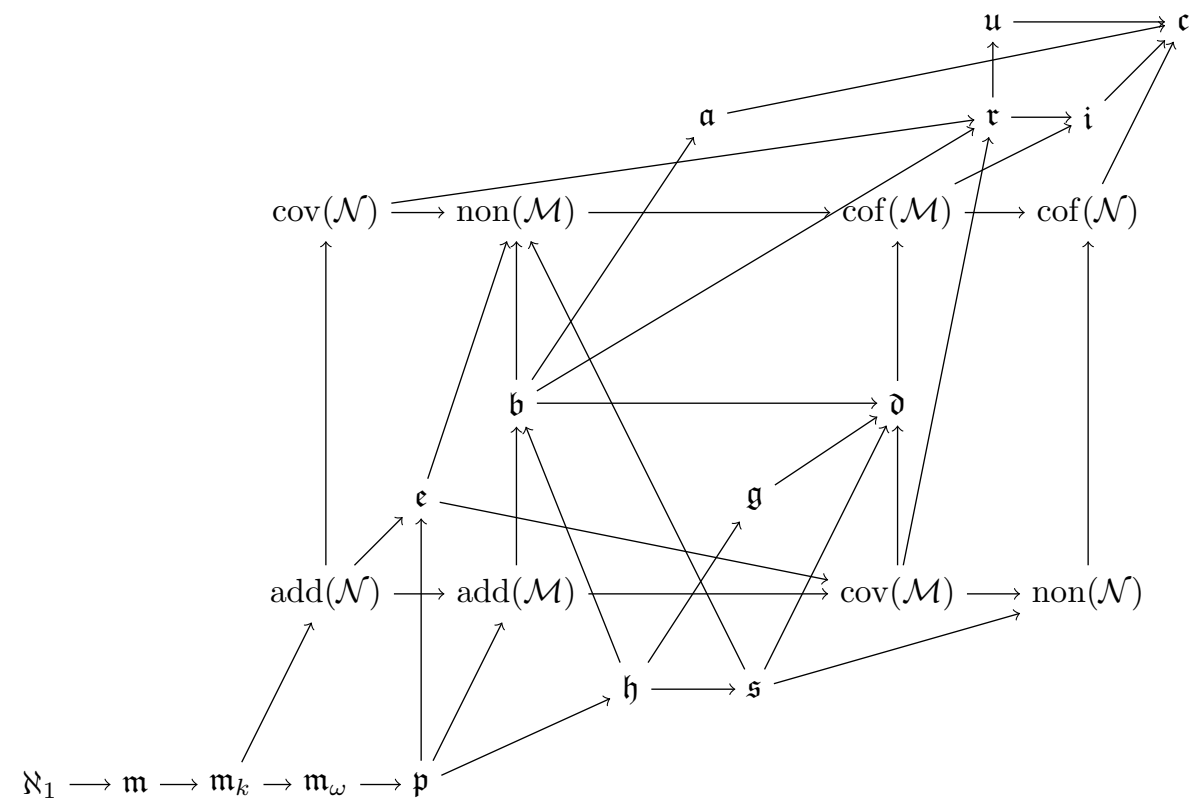

Figure 2. Cichoń's diagram and the Blass diagram combined.

An arrow $\mathfrak{x} \rightarrow \mathfrak{y}$ means that ZFC proves $\mathfrak{x} \leq \mathfrak{y}$.

Below we list some additional properties of these cardinals. Unless noted otherwise, proofs can be found in [Bla10].

FACT 1.2: (1) In [MS16] it was proved that $\mathfrak{p}=\mathfrak{t}^{2}$

(2) The cardinals $\operatorname{add}(\mathcal{N}), \operatorname{add}(\mathcal{M}), \mathfrak{b}, \mathfrak{t}, \mathfrak{h}$ and $\mathfrak{g}$ are regular.

(3) $\operatorname{cof}(\mathfrak{s}) \geq \mathfrak{t}($ see $[\mathrm{DS} 18])$.

(4) $2^{<\mathfrak{t}}=\mathfrak{c}$.

(5) $\operatorname{cof}(\mathfrak{c}) \geq \mathfrak{g}$.

(6) For $1 \leq k \leq k^{\prime} \leq \omega, \mathfrak{m}_{k} \leq \mathfrak{m}_{k^{\prime}}$.

(7) For $1 \leq k \leq \omega, \mathfrak{m}_{k}>\aleph_{1}$ implies $\mathfrak{m}_{k}=\mathfrak{m}_{\omega}$ (well-known but see, e.g., [GKMSb, Lemma 4.2]).

\footnotetext{
2 Only the trivial inequality $\mathfrak{p} \leq \mathfrak{t}$ is used in this text.
} 
This work contributes to the project of constructing a forcing model satisfying:

All the cardinals in Figure 2 are pairwise different,

with the obvious (ZFC provable) exception of the dependent entries $\operatorname{add}(\mathcal{M})=\min \{\mathfrak{b}, \operatorname{cov}(\mathcal{M})\}$ and $\operatorname{cof}(\mathcal{M})=\max \{\operatorname{non}(\mathcal{M}), \mathfrak{d}\}$, and the Martin axiom numbers $\mathfrak{m}, \mathfrak{m}_{k}$ for some $2 \leq k<\omega$, and $\mathfrak{m}_{\omega}$, which cannot have more than one value $>\aleph_{1}$; see Fact 1.2(7).

In this direction [GKS19] constructed a forcing model, using four strongly compact cardinals, where all the ten (non-dependent) values of Cichon's diagram are pairwise different (a situation we call Cichon's Maximum), as in Figure 3(A). This was improved later in [BCM21] by only using three strongly compact cardinals; finally in [GKMSa] it was shown that no large cardinals are needed for Cichoń's Maximum.

A model of Cichoń's Maximum with the order as in Figure 3(B) was obtained in [KST19]. Although this model initially required four strongly compact cardinals as well, the methods of [GKMSa] allow to remove the large cardinal assumptions also here.

As a next step towards ( $)$, [GKMSb] proved:

Theorem 1.3 ([GKMSb]): Under GCH, for any $k \in[1, \omega)$, there is a cofinality preserving poset $\mathbb{P}_{k}$ forcing that

(a) Cichon's Maximum holds with the order of Figure 3(A),

(b) $\aleph_{1}=\mathfrak{m}_{k-1}<\mathfrak{m}_{k}=\mathfrak{m}_{\omega}<\mathfrak{p}<\mathfrak{h}<\operatorname{add}(\mathcal{N})\left(\right.$ recall $\left.\mathfrak{m}_{0}:=\aleph_{1}\right)$.

An analogous result holds for the alternative order of Figure 3(в).

In this paper, we continue this line of work by including, in addition, $\mathfrak{s}$ and $\mathfrak{r}$. MAIN TheOREM: Under GCH, for any $k \in[2, \omega)$ there is a cofinality preserving poset which forces that the cardinals in Cichon's diagram, $\mathfrak{m}_{k}, \mathfrak{p}, \mathfrak{h}, \mathfrak{s}$ and $\mathfrak{r}$ are pairwise different. More specifically:

(a) Cichon's Maximum holds, in either of the orders of Figure 3.

(b) $\aleph_{1}=\mathfrak{m}_{k-1}<\mathfrak{m}_{k}=\mathfrak{m}_{\omega}<\mathfrak{p}<\mathfrak{h}<\operatorname{add}(\mathcal{N})$.

(c) $\mathfrak{s}$ can assume any regular value between $\mathfrak{p}$ and $\mathfrak{b}$.

(d) $\mathfrak{r}$ can assume any regular value in the dual position to $\mathfrak{s}$. Foe example, if $\mathfrak{s}<\operatorname{add}(\mathcal{N})$, then $\mathfrak{r}$ can be any arbitrary regular $\left[\operatorname{cof}(\mathcal{N}), \mathfrak{c}^{\text {fin }}\right]$ (see Section 7$)$.

In both theorems above, item (b) can also be replaced by

$$
\aleph_{1}<\mathfrak{m}_{\omega}<\mathfrak{p}<\mathfrak{h}<\operatorname{add}(\mathcal{N})
$$

while $\mathfrak{m}_{k}=\aleph_{1}$ for all $k<\omega$. Those are the only possible constellations of the Knaster numbers, by Fact 1.2(7), unless you count $\mathfrak{m}$ as the 1-Knaster-number: 
In contrast to Theorem 1.3 (where we do not control $\mathfrak{r}, \mathfrak{s}$ ), we cannot force $\mathfrak{m}>\aleph_{1}$ with the methods we use here. We cannot just iterate over all small ccc forcings one by one to increase $\mathfrak{m}$, as our method requires that all iterands of the forcing iteration have to be "homogeneous". So instead of using a certain small forcing $\dot{Q}$ as iterand, we will use a finite support product over all variants as iterand. So only if $\dot{Q}$ (and therefore all variants) is Knaster, ${ }^{3}$ this product can be used in a ccc iteration; accordingly we can increase the Knaster numbers but not $\mathfrak{m}$ itself.

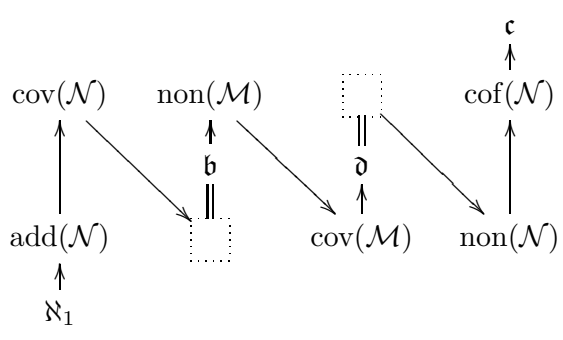

(A) [GKS19, GKMSa]

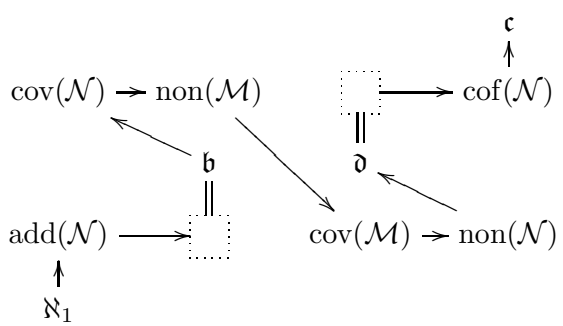

(B) $[\mathrm{KST} 19, \mathrm{GKMSa}]$

Figure 3. The two known consistent orders where all the (nondependent) values in Cichoń's diagram are pairwise different. (A) corresponds to the model in [GKS19], and (B) to the model in [KST19] (both proven consistent in [GKMSa] without large cardinals). Each arrow can be $<$ or $=$ as desired.

We remark that the full power of GCH is not required in the Main Theorem, but we do need some assumption on cardinal arithmetic in the ground model. See details in Section 7.

In order to include $\mathfrak{s}$ and $\mathfrak{r}$ in our main result, we need a new preservation theorem for splitting families. Previously, the following was known in the context of FS (finite support) iterations:

[BD85] Hechler forcing (for adding a dominating real) preserves splitting families witnessing the property $\mathrm{LCU}_{\mathbf{R}_{\mathrm{sp}}}(\kappa)$ for any uncountable regular $\kappa$ (see Section 3).

[JS88] Assuming CH, any FS iteration of Suslin ccc posets forces that the ground model reals form a splitting family.

\footnotetext{
3 Or at least stays ccc in ccc extensions.
} 
In this paper we will use a splitting family obtained by a FS product of Hechlertype posets (cf. [Hec72]) which we call $\mathbb{G}_{\mathbf{B}}$; the support of $\mathbb{G}_{\mathbf{B}}$ is a graph $\mathbf{B}$ of size $\aleph_{1}$ with certain homogeneity properties. We then show that this splitting family is preserved by certain FS iterations, which we will call "symmetric Suslin- $\lambda$-small". (Every FS iteration of Suslin ccc posets with parameters in the ground model is such an iteration, but our application will not use such "full" Suslin ccc forcings.)

Similar preservation techniques have appeared in different contexts. For instance, concerning preservation of mad (maximal almost disjoint) families, Kunen [Kun80] constructed, under CH, a mad family that can be preserved by Cohen posets; afterwards, Steprāns [Ste93] showed that, after adding $\omega_{1}$ many Cohen reals, there is a mad family of size $\aleph_{1}$ that can be preserved in further Cohen extensions; Fischer and Brendle [BF11] constructed a Hechlertype poset $\mathbb{H}_{A}$ with support (any uncountable set) $A$ that adds a mad family indexed by $A$, which can be preserved not only in further Cohen extensions but after other concrete FS iterations, thus generalizing Steprāns' result because $\mathbb{H}_{\omega_{1}}=\mathbb{C}_{\omega_{1}} ;[$ FFMM18, Mej19a] showed that any such mad family added by $\mathbb{H}_{A}$ can be preserved by some general type of FS iterations, but the most general result so far was shown in [BCM21]: Any $\kappa$-Fr-Knaster poset preserves $\kappa$-strong-Md-families (with $\kappa$ uncountable regular; the mad family added by $\mathbb{H}_{\kappa}$ is of such type).

There are deep technical differences between the mad family added by this $\mathbb{H}_{A}$, and the construction of a splitting family in this paper: No structure is needed on $A$, and because of this it is clear that Hechler's posets satisfy $\mathbb{H}_{A} \lessdot \mathbb{H}_{B}$ whenever $A \subseteq B$; but we cannot guarantee $\mathbb{G}_{\mathbf{B}_{0}} \lessdot \mathbb{G}_{\mathbf{B}}$ for our posets, whenever $\mathbf{B}_{0}$ is a subgraph of $\mathbf{B}$. Also, $\mathbb{G}_{\mathbf{B}}$ itself does not add a splitting family, but it just adds a set of Cohen reals $\left\{\eta_{a}: a \in \mathbf{B}\right\}$ over the ground model (recall that we do not have intermediate extensions by restricting the support $\mathbf{B}$ ). Hence, the FS product (or iteration, which is the same, as the poset $\mathbb{G}_{\mathbf{B}}$ is absolute) of size $\kappa$ of such posets adds a splitting family of size $\kappa$ (witnessing $\operatorname{LCU}_{\mathbf{R}}(\kappa)$ ) formed by the previously mentioned Cohen reals. It is clear that just adding $\kappa$ many Cohen reals produces a splitting family satisfying $\operatorname{LCU}_{\mathbf{R}}(\kappa)$, but we need to use FS support products of $\kappa$ many $\mathbb{G}_{\mathbf{B}}$ (with $\mathbf{B}$ of size $\aleph_{1}$, instead of just one $\mathbb{G}_{\mathbf{B}^{\prime}}$ with $\mathbf{B}^{\prime}$ of size $\kappa$ ), and we need the graph structure on $\mathbf{B}$, to be able to guarantee the preservation of the new splitting family. The forcing structure 
is very important here because an isomorphism of names argument is required for this preservation.

The strategy to prove the main theorem is similar to Theorem 1.3. We first show how to construct a ccc poset that forces distinct values for the cardinals on the left side of Cichon's diagram, including some of the other cardinal characteristics (like $\mathfrak{s}$ in this case). Afterwards, methods from [GKMSa, GKMSb] are applied to this initial forcing to get the poset for the main theorem.

\section{Annotated COntents.}

$\S 2$ We show how to construct, in ZFC, a suitable 2-graph. This is the type of graph we use as support for $\mathbb{G}_{\mathbf{B}}$.

$\S 3$ The LCU and COB properties are reviewed from [GKS19, GKMSa, GKMSb]. These describe strong witnesses to cardinal characteristics associated with a definable relation on the reals. Examples of such cardinal characteristics are the Cichoń-characteristics as well as $\mathfrak{s}$ and $\mathfrak{r}$.

$\S 4$ We introduce the forcing $\mathbb{G}_{\mathbf{B}}$, which has as support a suitable 2-graph $\mathbf{B}$. We look at FS iterations of ccc posets, in general, whose initial part is a FS product of posets of the form $\mathbb{G}_{\mathbf{B}}$ where $\mathbf{B}$ is in the ground model. We define $\lambda$-small history iterations (where on a dense set, conditions have $<\lambda$-sized history), as well as symmetric iterations, and show that symmetric $\lambda$-small history iterations allow us to control $\mathfrak{s}$ (and later also $\mathfrak{r}$ ).

$\S 5$ We define Suslin $\lambda$-small iterations, which are $\lambda$-small history iterations, and give consequences of these notions, as well as sufficient conditions to get symmetric ones.

$\S 6$ Closely following [GKS19], we construct a symmetric Suslin- $\lambda$-small iteration $\mathbb{P}^{0}$ that separates the cardinals on the left-hand side of the diagram, with $\operatorname{cov}(\mathcal{M})=\mathfrak{c}$ and $\mathfrak{s}=\mathfrak{p}$.

$\S 7$ We show how the tools of [GKMSa, GKMSb] can be applied to $\mathbb{P}^{0}$, resulting in a forcing that gives the main theorem.

$\S 8$ We discuss some open questions related to this work. 


\section{Suitable 2-graphs}

In this section we define and construct suitable 2-graphs.

Definition 2.1: Say that $\mathbf{B}:=\left\langle B, R_{0}, R_{1}\right\rangle$ is a 2-edge-labeled graph, abbreviated 2-graph, if

(i) $R_{0}$ and $R_{1}$ are irreflexive symmetric relations on $B$,

(ii) $R_{0} \cap R_{1}=\emptyset$.

In other words: Between two nodes $x$ and $y$ there is at most one edge, with color 0 or 1.

Concerning 2-graphs, we define the following notions:

(1) If $A \subseteq B$, denote $\left.\mathbf{B}\right|_{A}:=\left\langle A,\left.R_{0}\right|_{A},\left.R_{1}\right|_{A}\right\rangle$ where $\left.R_{e}\right|_{A}:=R_{e} \cap(A \times A)$.

(2) A partial function (or coloring) $\eta$ from $B$ into 2 respects $\mathbf{B}$ if

$$
\{\eta(a), \eta(b)\} \neq\{e\}
$$

whenever $e \in 2, a, b \in \operatorname{dom} \eta$ and $a R_{e} b$.

The 2-graph of Figure 4 does not have a coloring (with full domain) respecting it.

A 2-graph $\mathbf{B}$ is a suitable 2-graph $(\mathbf{S 2 G})$ if it satisfies, in addition,

(iii) $|B|=\aleph_{1}$,

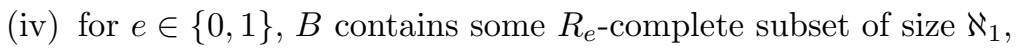

(v) if $a \in B$ and $e \in\{0,1\}$ then there is some $\eta: B \rightarrow 2$ respecting $\mathbf{B}$ such that $\eta(a)=e$.

(vi) For any $a, b \in B$, there is some automorphism $f$ of $\mathbf{B}$ such that $f(a)=b$.

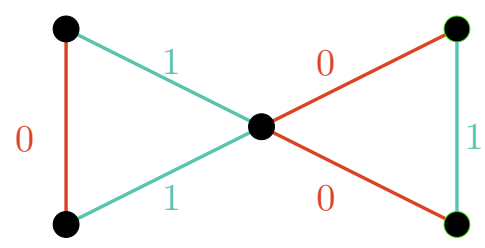

Figure 4. A finite 2-graph which cannot be respected by any coloring.

Properties (iv) and (vi) imply for all $b \in B$ and $e \in\{0,1\}$ :

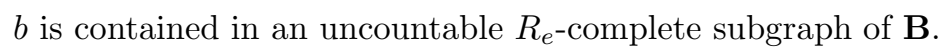


In the rest of this section we will show that an S2G exists. ${ }^{4}$

Remark 2.3: In our applications, we only need the following weakening of prop$\operatorname{erty}(\mathrm{v})$ : for any $t \in[B]^{<\aleph_{0}}, a \in t$ and $e \in 2$, there is some $\eta: t \rightarrow 2$ that respects $\mathbf{B}$ such that $\eta(a)=e$. The only place where (the weakening of) (v) is used is in the proof of Lemma $4.2(\mathrm{~b})$.

Definition 2.4: Fix a 2-graph B.

(1) A finite partial function $s: B \rightarrow 2$ with $|\operatorname{dom} s| \geq 2$ (which we may also call "finite positive atomic type") is realized by $z \in B$, if $x R_{s(x)} z$ for any $x \in \operatorname{dom} s$.

(2) Let $D \subseteq B$. We say $D \preceq-B$ if any such type $s: D \rightarrow 2$ which is realized in $B$ is also realized in $D$.

Note that we require this only for "types" with at least two edges. So when checking $D \preceq-B$ we can ignore all $b \in B$ which have at most one edge to elements of $D$.

Lemma 2.5: Let $\mathbf{B}=\left\langle B, R_{0}, R_{1}\right\rangle$ be a 2-graph and $A \preceq^{-} B$. Then:

(a) If $\eta: A \rightarrow 2$ respects $\mathbf{B}$ and $c \in B \backslash A$, then $\eta$ can be extended to some $\eta^{\prime}: A \cup\{c\} \rightarrow 2$ that respects $\mathbf{B}$.

(b) If in addition $\left.\mathbf{B}\right|_{A}$ satisfies (v) of Definition 2.1 then, whenever $c \in B \backslash A$ and $e \in 2$, there is some $\eta^{\prime}: A \cup\{c\} \rightarrow 2$ that respects $\mathbf{B}$ such that $\eta^{\prime}(c)=e$.

(c) Now assume that all elements of $B \backslash A$ have edges only to elements of $A$, i.e., $(B \backslash A)^{2} \cap\left(R_{0} \cup R_{1}\right)=\emptyset$. Then under the assumptions of (a), we can extend $\eta$ to some $\eta^{\prime \prime}: B \rightarrow 2$; and under $(b)$ we can find some $\eta^{\prime \prime}: B \rightarrow 2$ with $\eta^{\prime \prime}(c)=e$.

Proof. (a): By contradiction: Assume that $\eta$ cannot be extended in such a way, which means that there are $x \neq y$ in $A$ such that $x R_{0} c, y R_{1} c, \eta(x)=0$ and $\eta(y)=1$. Since $A \preceq^{-} B$, there is some $z \in A$ such that $x R_{0} z$ and $y R_{1} z$, but this contradicts that $\eta$ respects $\mathbf{B}$.

\footnotetext{
${ }^{4}$ Using saturation, it is easy to see that it is enough to show the weaker result that a "weak $\mathrm{S} 2 \mathrm{G}$ " exists, where for "weak $\mathrm{S} 2 \mathrm{G}$ " we replace the requirement that the three sets $(B$ and the two complete subsets) have size $\aleph_{1}$, with the requirement that they are infinite.

We could then work in $L$, use the fact that a weak S2G exists and find an $\omega_{1}^{V}$-saturated model $\mathbf{B}$ for it; due to saturation the three sets have size $\omega_{1}^{V}$; and by absoluteness $\mathbf{B}$ is actually an S2G in $V$.

However, as the construction for a weak S2G is not any easier than the construction for $\mathrm{S} 2 \mathrm{G}$, we do not use saturation.
} 
(b): Assume $x_{0} R_{1-e} c$ for some $x_{0} \in A$. Then, by Definition 2.1(v), there is some $\eta: A \rightarrow 2$ respecting $\mathbf{B}$ such that $\eta\left(x_{0}\right)=1-e$. By (a) we can extend it to $\eta^{\prime}: A \cup\{c\} \rightarrow 2$, and $\eta^{\prime}(c)$ has to be $e$.

So now we assume that $c$ only has $e$-connections to $A$ (if any). It is enough to show that there is some $\eta$ coloring $A$ which assigns $1-e$ to all neighbours of $c$ in $A$ : Then we can again extend it by setting $\eta^{\prime}(c)=e$.

Say that $p: w_{p} \rightarrow A$ is a 0 -1-path if it satisfies

(i) $0<w_{p} \leq \omega$,

(ii) $p(0) R_{e} c$,

(iii) if $n<w_{p}$ and $n \equiv i \bmod 2$ then $p(n) R_{|1-e-i|} p(n+1)$, that is,

$$
p(0) R_{1-e} p(1) R_{e} p(2) R_{1-e} p(3) \cdots .
$$

It is clear that, whenever $p: w_{p} \rightarrow A$ is a $0-1$ path, then there is a unique $\eta_{p}: \operatorname{ran} p \rightarrow 2$ respecting $\mathbf{B}$ such that $\eta_{p}(p(0))=1-e$. Some such coloring exists, as we assume Definition 2.1(v) for $\left.\mathbf{B}\right|_{A}$. Uniqueness is clear: as $p(0)$ gets color $1-e, p(1)$ has to have color $e$, etc., i.e. $\eta_{p}(p(n))=e$ iff $n$ is odd. Note that (2.6) $\eta_{p}(p(n))=j$ implies that the edge from $p(n)$ to $p(n-1)$ has color $1-j$

(where we set $p(-1):=c$ ).

Let $A^{\prime} \subseteq A$ be the union of (the ranges of) all 0-1-paths. We first show that there is a unique $\eta_{c}: A^{\prime} \rightarrow 2$ respecting $\mathbf{B}$ such that $\eta_{c}(x)=1-e$ whenever $x R_{e} c$.

Uniqueness is clear: Each node in $A^{\prime}$ lies on a 0-1-path, which determines its color. Set $\eta_{c}$ to be the union of the $\eta_{p}$ for all 0-1-paths $p$. So it is enough to show that $\eta_{c}$ is a function and that it respects $\mathbf{B}$.

$\eta_{c}$ is a function: Assume that $p, q$ are 0-1-paths, $m<w_{p}$ and $n<w_{q}$ and $p(m)=q(n)$. If $p(0)=q(0)$ then there is some $\eta_{0}: A \rightarrow 2$ respecting $\mathbf{B}$ with $\eta_{0}(p(0))=1-e$, and this $\eta_{0}$ must extend both $\eta_{p}$ and $\eta_{q}$, hence

$$
\eta_{p}(p(m))=\eta_{q}(q(n))=\eta_{0}(p(m)) .
$$

So assume $p(0) \neq q(0)$, so in particular $p(0) R_{e} c$ and $q(0) R_{e} c$. As $A \preceq^{-} B$, there is some $z \in A$ such that $p(0) R_{e} z$ and $q(0) R_{e} z$. We can choose $\eta_{1}: A \rightarrow 2$ respecting $\mathbf{B}$ such that $\eta_{1}(z)=e$. This implies $\eta_{1}(p(0))=\eta_{1}(q(0))=1-e$, hence $\eta_{1}$ extends both $\eta_{p}$ and $\eta_{q}$, so

$$
\eta_{p}(p(m))=\eta_{q}(q(n))=\eta_{1}(p(m)) .
$$


$\eta_{c}$ respects B: Assume towards a contradiction that there are 0-1-paths $p, q, m<w_{p}, n<w_{q}$ and $j<2$ such that $\eta_{c}(p(m))=\eta_{c}(q(n))=j$ and $p(m) R_{j} q(n)$. It cannot be that $p(0)=q(0)$ because there is some $\eta_{0}: A \rightarrow 2$ respecting $\mathbf{B}$ with $\eta_{0}(p(0))=1-e$, and such $\eta_{0}$ must extend both $\eta_{p}$ and $\eta_{q}$. Hence $p(0) \neq q(0)$. As before, $A \preceq-B$ implies that there is some $z \in A$ such that $p(0) R_{e} z$ and $q(0) R_{e} z$, and there is an $\eta_{1}: A \rightarrow 2$ respecting $\mathbf{B}$ such that $\eta_{1}(z)=e$. Since $\eta_{1}$ extends both $\eta_{p}$ and $\eta_{q}, \eta_{1}(p(m))=\eta_{1}(q(n))=j$ and $p(m) R_{j} q(n)$, a contradiction.

It remains to be shown that $\eta_{c}$ can be extended to all of $A$. For this, choose any $\eta_{2}: A \rightarrow 2$ respecting $\mathbf{B}$, and define $\eta: A \rightarrow 2$ by $\eta(x):=\eta_{c}(x)$ if $x \in A^{\prime}$, or $\eta(x):=\eta_{2}(x)$ otherwise. We claim that $\eta$ respects B. Assume otherwise, i.e., there are $x \in A^{\prime}, y \in A \backslash A^{\prime}$ and $j<2$ such that $x R_{j} y$ and $\eta(x)=\eta(y)=j$. Since $x \in A^{\prime}$, there is some 0-1-path $p$ such that $x=p(n)$. By $(2.6) \eta_{c}(x)=j$ implies that the edge between $p(n)$ and $p(n-1)$ is $1-j$, but this means that we can extend $p\left\lceil n\right.$ to $y$ and get another 0-1-path, a contradiction to $y \notin A^{\prime}$.

(c): First note that, whenever $A \subseteq A^{\prime} \subseteq B, A^{\prime} \preceq-B$ : Assume $b \in B \backslash A^{\prime}$. As there are no connections between $b$ and $A^{\prime} \backslash A$, any finite type over $A^{\prime}$ realized by $b$ is a type over $A$, which is realized in $A$ as $A \preceq^{-} B$.

Therefore, we can construct $\eta^{\prime \prime}$ by Zorn's Lemma or by induction (starting with a suitable $\eta^{\prime}$ as in (b), if required).

THEOREM 2.7: There exists a suitable 2-graph.

Proof. We are going to construct, using forcing notation, ${ }^{5}$ two relations $R_{0}^{*}$ and $R_{1}^{*}$ on $\omega_{1}$ such that $\left\langle\omega_{1}, R_{0}^{*}, R_{1}^{*}\right\rangle$ becomes a $\mathrm{S} 2 \mathrm{G}$. Define the poset $\mathbb{P}$ whose conditions are tuples $p=\left\langle B^{p}, R_{0}^{p}, R_{1}^{p}, W_{0}^{p}, W_{1}^{p}, L^{p}, A^{p}\right\rangle$ satisfying the following: (C1) $\mathbf{B}^{p}:=\left\langle B^{p}, R_{0}^{p}, R_{1}^{p}\right\rangle$ is a 2-graph with $B^{p} \subseteq \omega_{1}$ countable.

(C2) For each $e \in 2, W_{e}^{p} \subseteq B^{p}$ is infinite and $R_{e}^{p}$-complete, and $W_{0}^{p} \cap W_{1}^{p}=\emptyset$.

(C3) $L^{p}=\left\{\eta_{a, e}^{p}:(a, e) \in B^{p} \times 2\right\}$ where $\eta_{a, e}^{p}: B^{p} \rightarrow 2$ respects $\mathbf{B}^{p}$ and $\eta_{a, e}^{p}(a)=e$, for any $(a, e) \in B^{p} \times 2$.

(C4) $A^{p}=\left\{f_{a, b}^{p}:(a, b) \in C^{p}\right\}$ such that $C^{p} \subseteq B^{p} \times B^{p}$ and, for any $(a, b) \in C^{p}$ :

(F1) $f_{a, b}^{p}: D_{a, b}^{p} \rightarrow D_{a, b}^{p}$ is a bijection,

(F2) $a, b \in D_{a, b}^{p}$ and $f_{a, b}^{p}(a)=b$,

(F3) for any $x, y \in D_{a, b}^{p}$ and $e \in 2, x R_{e}^{p} y$ iff $f_{a, b}^{p}(x) R_{e}^{p} f_{a, b}^{p}(y)$,

(F4) $D_{a, b}^{p} \preceq-B^{p}$.

5 Equivalently we could formulate it as an inductive construction, taking care of $\aleph_{1}$-many requirements in $\omega_{1}$-many steps. 
Order $\mathbb{P}$ by $q \leq p$ iff the following is satisfied:

(O1) $\mathbf{B}^{p}$ is a 2-subgraph of $\mathbf{B}^{q}$, and $B^{p} \preceq^{-} B^{q}$,

(O2) $C^{p} \subseteq C^{q}$ and $W_{e}^{q} \cap B^{p}=W_{e}^{p}$ for $e \in 2$,

(O3) for any $a \in B^{p}$ and $e \in 2, \eta_{a, e}^{p} \subseteq \eta_{a, e}^{q}$, and

(O4) for any $(a, b) \in C^{p}, f_{a, b}^{p} \subseteq f_{a, b}^{q}$.

Note that $\mathbb{P} \neq \emptyset$. Indeed, choose disjoint $W_{0}^{\bullet}, W_{1}^{\bullet} \subseteq \omega_{1}$ of size $\aleph_{0}$, and define $\mathbf{B}^{\bullet}:=\left\langle B^{\bullet}, R_{0}^{\bullet}, R_{1}^{\bullet}\right\rangle$ where $B^{\bullet}:=W_{0}^{\bullet} \cup W_{1}^{\bullet}$ and, for $e \in 2, x R_{e}^{\bullet} y$ iff $x, y \in W_{e}^{\bullet}$. It is easy to construct an $L^{\bullet}$ such that $\left\langle B^{\bullet}, R_{0}^{\bullet}, R_{1}^{\bullet}, W_{0}^{\bullet}, W_{1}^{\bullet}, L^{\bullet}, \emptyset\right\rangle$ is a condition in $\mathbb{P}$.

Recall that, for any $\sigma$-closed poset and arbitrary $\aleph_{1}$-many dense subsets, there is a filter intersecting these dense sets. So, after showing that $\mathbb{P}$ is $\sigma$-closed, we can obtain a suitable 2-graph from a filter intersecting suitable dense sets.

$\mathbb{P}$ IS $\sigma$-CLOSED: Let $\left\langle p_{n}: n<\omega\right\rangle$ be a decreasing sequence of conditions in $\mathbb{P}$. Denote $B^{p_{n}}=B^{n}, R_{0}^{p_{n}}=R_{0}^{n}$, and so on. Set $B:=\bigcup_{n<\omega} B^{n}, R_{e}:=\bigcup_{n<\omega} R_{e}^{n}$ and $W_{e}:=\bigcup_{n<\omega} W_{e}^{n}$ for $e \in\{0,1\}, \mathbf{B}:=\left\langle B, R_{0}, R_{1}\right\rangle$, and $C:=\bigcup_{n<\omega} C^{n}$. For $(a, b) \in C$ set

$$
f_{a, b}:=\bigcup_{n \geq m} f_{a, b}^{n}
$$

where $m=\min \left\{n<\omega:(a, b) \in C^{n}\right\}$. For $a \in B$ and $e \in 2$, set

$$
\eta_{a, e}:=\bigcup_{n \geq m} \eta_{a, e}^{n}
$$

where $m=\min \left\{n<\omega: a \in B^{n}\right\}$. Put

$$
\begin{gathered}
L:=\left\{\eta_{a, e}:(a, e) \in B \times 2\right\}, \quad A:=\left\{f_{a, b}:(a, b) \in C\right\}, \\
\text { and } q:=\left\langle B, R_{0}, R_{1}, W_{0}, W_{1}, L, A\right\rangle .
\end{gathered}
$$

It is easy to see that $q \in \mathbb{P}$ and that it is stronger than each $p_{n}$.

The following sets are dense in $\mathbb{P}$ :

(I) $D_{a^{*}}:=\left\{p \in \mathbb{P}: a^{*} \in B^{p}\right\}$ for any $a^{*} \in \omega_{1}$.

Let $p \in \mathbb{P}$ and assume $a^{*} \notin B^{p}$. We define $q \leq p$ in $D_{a^{*}}$ as follows:

(i) $B^{q}:=B^{p} \cup\left\{a^{*}\right\}$;

(ii) $R_{e}^{q}:=R_{e}^{p}$ and $W_{e}^{q}:=W_{e}^{p}$ for $e \in\{0,1\}$;

(iii) $C^{q}:=C^{p}$;

(iv) $f_{a, b}^{q}:=f_{a, b}^{p}$ for all $(a, b) \in C^{p}$.

Obviously we can extend each old $\eta_{a, e}^{q}$ (by assigning an arbitrary value $e$ to $a^{*}$ ), and picking two such extensions for $e=0,1$ we get the required $\eta_{a^{*}, e}^{q}$. 
It is clear that $B^{p} \preceq^{-} B^{q}$, as the new node has no edges. This implies that

$$
D_{(a, b)}^{q}=D_{(a, b)}^{p} \preceq^{-} B^{q},
$$

as $\preceq^{-}$is transitive (the same argument will apply to the following dense sets as well).

(II) $E_{a^{*}, b^{*}}:=\left\{p \in \mathbb{P}:\left(a^{*}, b^{*}\right) \in C^{p}\right\}$ for any $a^{*}, b^{*} \in \omega_{1}$.

Without loss of generality assume that $p \in \mathbb{P}$ and $a^{*}, b^{*} \in B^{p}$, but $\left(a^{*}, b^{*}\right) \notin C^{p}$. We want to find some $q \leq p$ in $E_{a^{*}, b^{*}}$. When $a^{*}=b^{*}$, it is enough to set $f_{a^{*}, b^{*}}^{q}=\operatorname{id}_{B^{p}}, C^{q}:=C^{p} \cup\left\{\left(a^{*}, a^{*}\right)\right\}$, and leave the other components as in $p$. So assume that $a^{*} \neq b^{*}$.

The set $B^{q}$ will be the union of $\mathbb{Z}$ many copies of $B^{p}$, where $b^{*}$ in the $m$-th copy is identified with $a^{*}$ in the $m+1$-th copy. In more detail: Denote $B_{0}:=B^{p}$. Find a sequence $\left\langle B_{m}^{\prime}: m \in \mathbb{Z} \backslash\{0\}\right\rangle$ of pairwise disjoint subsets of $\omega_{1}$ of size $\aleph_{0}$ and disjoint to $B_{0}$. Set $a_{0}:=a^{*}, a_{1}:=b^{*}$, and for $m \in \mathbb{Z} \backslash\{0,1\}$ choose pairwise different $a_{m} \in \omega_{1} \backslash\left(B_{0} \cup \bigcup_{m \in \mathbb{Z} \backslash\{0\}} B_{m}^{\prime}\right)$. For $m \neq 0$ put

$$
B_{m}:=B_{m}^{\prime} \cup\left\{a_{m}, a_{m+1}\right\} .
$$

Note that, for any $m, n \in \mathbb{Z}$, if $|m-n|>1$ then

$$
B_{m} \cap B_{n}=\emptyset \quad \text { and } \quad B_{m} \cap B_{m+1}=\left\{a_{m+1}\right\} .
$$

Choose a bijection $g_{m}: B_{m} \backslash\left\{a_{m+1}\right\} \rightarrow B_{m+1} \backslash\left\{a_{m+2}\right\}$ such that $g_{m}\left(a_{m}\right)=a_{m+1}$, and let

$$
f_{a^{*}, b^{*}}^{q}=f:=\bigcup_{m \in \mathbb{Z}} g_{m}
$$

which is a bijection from

$$
B^{q}:=\bigcup_{m \in \mathbb{Z}} B_{m}
$$

onto itself. Define for $e \in 2$ and $x, y \in B^{q}, x R_{e}^{q} y$ iff:

$x \neq y$, they belong to the same (unique) $B_{m}$ and $f^{(-m)}(x) R_{e}^{p} f^{(-m)}(y)$.

It is clear that $B^{p}=B_{0} \preceq^{-} B^{q}$, as any $x \in B^{q} \backslash B^{p}$ has connections to at most one node in $B^{p}$ (to either $a^{*}$ or $b^{*}$ ).

We set $W_{e}^{q}:=W_{e}^{p}$ and $C^{q}:=C^{p} \cup\left\{\left(a^{*}, b^{*}\right)\right\}$, and leave the partial automorphisms in $p$ unchanged, i.e., $f_{a, b}^{q}:=f_{a, b}^{p}$ for $(a, b) \in C^{p}$.

It is now enough to show that we can extend every old $\eta_{a, e}^{p}$ to $\eta_{a, e}^{q}: B^{q} \rightarrow 2$, and find for each new $b \in B^{q}$ and $e \in 2$ a suitable $\eta_{b, e}^{q}$. 
Assume $\eta_{0}:=\eta_{a, e}^{p} \in L^{p}$. We extend $\eta_{0}$ in the following way: Let $e_{1}:=\eta_{a, e}^{p}\left(a_{1}\right)$. Set $\eta_{1}:=\eta_{a_{1}, e_{1}}^{p} \in L^{p}$. We now extend $\eta_{0}$ to $B_{1}$ by setting $\eta(f(x)):=\eta_{1}(x)$ for $x \in B_{0}$; and continue by induction (to the right and also to the left). In more detail: define $\eta_{n}: B_{n} \rightarrow 2$ and $\eta_{-n}: B_{-n} \rightarrow 2$ by recursion on $n \in \omega$ as $\eta_{n+1}:=\eta_{a, \eta_{n}\left(a_{n+1}\right)}^{p} \circ f^{-(n+1)}$ and $\eta_{-(n+1)}:=\eta_{b, \eta_{-n}\left(a_{-n}\right)}^{p} \circ f^{n+1}$ (we already have $\eta_{0}$ from the start). All these functions are compatible, so we can define

$$
\eta_{a, e}^{q}:=\bigcup_{m \in \mathbb{Z}} \eta_{m}
$$

and it is clear that it respects $\mathbf{B}^{q}$.

Similarly, we get new $\eta_{a, e}^{q}$ for $a \in B^{q} \backslash B^{p}$. Concretely,

$$
\eta_{a, e}^{q}:=\eta_{f-m}^{q}(a), e^{\circ} f^{-m}
$$

where $m$ is the one with minimum absolute value such that $a \in B_{m}$, and $\eta_{f^{-m}(x), e}^{q}$ is defined as in the previous paragraph.

(III) $E_{a^{*}, b^{*}, c^{*}}^{\prime}:=\left\{p \in \mathbb{P}:\left(a^{*}, b^{*}\right) \in C^{p}, c^{*} \in D_{a^{*}, b^{*}}^{p}\right\}$ for any $a^{*}, b^{*}, c^{*} \in \omega_{1}$.

Without loss of generality, assume $p \in \mathbb{P},\left(a^{*}, b^{*}\right) \in C^{p}$ and $c^{*} \in B^{p} \backslash D_{a^{*}, b^{*}}^{p}$. Denote $D^{p}:=D_{a^{*}, b^{*}}^{p}$. Let $c_{0}:=c^{*}$ and, for $m \in \mathbb{Z} \backslash\{0\}$, choose pairwise different $c_{m} \in \omega_{1} \backslash B^{p}$. Set $D^{q}:=D^{p} \cup\left\{c_{m}: m \in \mathbb{Z}\right\}$ and $f: D^{q} \rightarrow D^{q}$ extending $f_{a^{*}, b^{*}}^{p}$ such that $f\left(c_{m}\right):=c_{m+1}$. Define $q$ as follows:

(i) $B^{q}:=B^{p} \cup\left\{c_{m}: m \in \mathbb{Z} \backslash\{0\}\right\}$;

(ii) a new node $c_{n}$ has an $R_{e}$-edge to $f^{n}(x)$ iff $c_{0}=c^{*}$ has an $R_{e}$-edge to $x$;

(iii) $W_{e}^{q}:=W_{e}^{p}$;

(iv) $C^{q}:=C^{p}$;

(v) $f_{a^{*}, b^{*}}^{q}=f$ (with $D_{a^{*}, b^{*}}^{q}=D^{q}$ ), and the other partial automorphisms are unchanged (i.e., for $\left.(a, b) \in C^{q} \backslash\left\{\left(a^{*}, b^{*}\right)\right\}, f_{a, b}^{q}:=f_{a, b}^{p}\right)$.

$B^{p} \preceq^{-} B^{q}$ : Let $s: B^{p} \rightarrow 2$ be a type realized by $c_{n}(n \neq 0)$. Then actually $\operatorname{dom}(s) \subseteq D^{p}$, as $c_{n}$ only has connections to $D^{p}$. As $D^{p} \preceq^{-} B^{p}$, and the type $s^{\prime}:=s \circ f^{n}$ is realized by $c^{*}=c_{0}$, we know that $s^{\prime}$ is realized by some $z \in D^{p}$. Then $s$ is realized by $f^{n}(z) \in D^{p} \subseteq B^{p}$.

$D_{a^{*}, b^{*}}^{q}=D^{q} \preceq^{-} B^{q}$ : If $x \in B^{q} \backslash D^{q}$, then $x \in B^{p}$ and has edges only to $B^{p}$. So any $s: D^{q} \rightarrow 2$ realized by $x$ has domain in $D^{p}$, and as $D^{p} \preceq^{-} B^{p}$, this $s$ is realized in $D^{p} \subseteq D^{q}$.

To see that we can extend all old $\eta_{a, e}^{p}$ to $\eta_{a, e}^{q}: B^{q} \rightarrow 2$, and that we can find $\eta_{c_{m}, e}^{q}$ for $e<2$ and $m \neq 0$, it is enough to note that all the assumptions in Lemma 2.5(c) are met (where we use $A=B^{p}$ and $B=B^{q}$ ). 
(IV) $E_{\alpha, e}^{\prime \prime}:=\left\{p \in \mathbb{P}: \exists b^{*} \in W_{e}^{p}\left(b^{*} \geq \alpha\right)\right\}$ for $\alpha<\omega_{1}$ and $e \in 2$.

Choose $b^{*} \in \omega_{1} \backslash\left(B^{p} \cup \alpha\right)$ and define $q$ such that

(i) $B^{q}:=B^{p} \cup\left\{b^{*}\right\}$, and the new node $b^{*}$ is $R_{e}$-connected to exactly the nodes in $W_{e}^{p}$, and has no $R_{1-e^{-c o n n e c t i o n s, ~}}$

(ii) $W_{e}^{q}:=W_{e}^{p} \cup\left\{b^{*}\right\}$ and $W_{1-e}^{q}:=W_{1-e}^{p}$,

(iii) $C^{q}:=C^{p}$,

(iv) $f_{a, b}^{q}:=f_{a, b}^{p}$ for all $(a, b) \in C^{p}$.

$B^{p} \preceq^{-} B^{q}$ : Let $s: B^{p} \rightarrow 2$ be realized by $b^{*}$. This implies that $\operatorname{dom} s \subseteq W_{e}^{p}$ and $s(x)=e$ for all $x \in \operatorname{dom} s$. Since $W_{e}^{p}$ is infinite, there is some $z \in W_{e}^{p} \subseteq B^{p}$ such that $x R_{e}^{p} z$ for all $x \in \operatorname{dom} s$.

Given any old $\eta_{a, e}^{p}$, we can extend it to a function $\eta_{a, e}^{q}$ with domain $B^{q}$ by Lemma 2.5(a); and for arbitrary $e \in 2$, we get $\eta_{b^{*}, e}^{q}$ by Lemma 2.5(b). (Again, we use $A=B^{p}$ and $B=B^{q}$.)

Let $\mathcal{D}$ be the collection of all dense sets defined above. Since $\mathbb{P}$ is $\sigma$-closed and $|\mathcal{D}|=\aleph_{1}$, there is some filter $G \subseteq \mathbb{P}$ intersecting all the dense sets in $\mathcal{D}$. Set $R_{e}^{*}:=\bigcup_{p \in G} R_{e}^{p}$ and $U_{e}:=\bigcup_{p \in G} W_{e}^{p}$ for $e \in\{0,1\}$. Since $G \cap D_{a} \neq \emptyset$ and $G \cap E_{a, b} \neq \emptyset$ for any $a, b \in \omega_{1}$, we have

$$
\bigcup_{p \in G} B^{p}=\omega_{1} \quad \text { and } \bigcup_{p \in G} C^{p}=\omega_{1} \times \omega_{1}
$$

Set $\mathbf{B}:=\left\langle\omega_{1}, R_{0}^{*}, R_{1}^{*}\right\rangle$, which is a 2-graph. It is clear that $\mathbf{B}^{p}$ is a 2 -subgraph of $\mathbf{B}$ for any $p \in G$. On the other hand, $E_{\alpha, e}^{\prime \prime} \cap G \neq \emptyset$ for all $\alpha<\omega_{1}$ and $e \in 2$, which implies that $U_{e}$ is an $R_{e}^{*}$-complete subset of $\omega_{1}$ of size $\aleph_{1}$. Even more, $U_{e} \cap B^{p}=W_{e}^{p}$ for any $p \in G$, and $U_{0} \cap U_{1}=\emptyset$.

For $a \in \omega_{1}$ and $e \in 2$ set

$$
\eta_{a, e}:=\bigcup\left\{\eta_{a, e}^{p}: a \in B^{p}, p \in G\right\} .
$$

It is routine to check that $\eta_{a, e}: \omega_{1} \rightarrow 2$ respects $\mathbf{B}$. This guarantees (v) of Definition 2.1.

For $a, b \in \omega_{1}$, set

$$
f_{a, b}:=\bigcup\left\{f_{a, b}^{p}:(a, b) \in C^{p}, p \in G\right\} .
$$

Since $G \cap E_{a, b, c}^{\prime} \neq \emptyset$ for any $c \in \omega_{1}, \bigcup_{p \in G} D_{a, b}^{p}=\omega_{1}$ and $f_{a, b}$ is a Bautomorphism. This shows property (vi) of Definition 2.1. Therefore, $\mathbf{B}$ is a $\mathrm{S} 2 \mathrm{G}$. 


\section{Cardinal characteristics, COB and LCU}

Many classical characteristics can be defined by the framework of relational systems as in, e.g., [Voj93, Bla10]. Say that $\mathbf{R}:=\langle X, Y, R\rangle$ is a relational system if $X$ and $Y$ are non-empty sets, and $R$ is a relation. The following cardinal characteristics are associated with $\mathbf{R}$ :

$$
\begin{aligned}
& \mathfrak{d}(\mathbf{R}):=\min \{|D|: D \subseteq Y \text { and } \forall x \in X \exists y \in D(x R y)\} ; \\
& \mathfrak{b}(\mathbf{R}):=\min \{|F|: F \subseteq X \text { and } \neg \exists y \in Y \forall x \in X(x R y)\} .
\end{aligned}
$$

In this work, we are particularly interested in relational systems $\mathbf{R}$ such that (RS1) $X$ and $Y$ are subsets of Polish spaces $Z_{0}$ and $Z_{1}$, respectively, and absolute for transitive models of ZFC (e.g. they are analytic);

(RS2) $R \subseteq Z_{0} \times Z_{1}$ is absolute for transitive models of ZFC (e.g., analytic in $Z_{0} \times Z_{1}$ ).

When these properties hold we say that $\mathbf{R}$ is a relational system of the reals. In all the cases explicitly mentioned throughout this paper, $X$ and $Y$ are Polish spaces themselves and $R$ is Borel in $X \times Y$. In this case, there is no problem to identify $X=Y=\omega^{\omega}$, and we call $\mathbf{R}$, or rather the characteristics $\mathfrak{b}(\mathbf{R})$ and $\mathfrak{d}(\mathbf{R})$, Blass-uniform (cf. [GKMSb, §2]).

Example 3.1 ([Voj93, 2.2.2] or [Bla10, §4 \& §5]): The splitting number $\mathfrak{s}$ and the reaping number $\mathfrak{r}$ are Blass-uniform: Denote $\mathbf{R}_{\mathrm{sp}}:=\left\langle 2^{\omega},[\omega]^{\aleph_{0}}, R_{\mathrm{sp}}\right\rangle$ where $x R_{\mathrm{sp}} y$ iff $x\left\lceil y\right.$ is constant except in finitely many points of $y$. Then $\mathfrak{s}=\mathfrak{b}\left(\mathbf{R}_{\mathrm{sp}}\right)$ and $\mathfrak{r}=\mathfrak{d}\left(\mathbf{R}_{\mathrm{sp}}\right){ }^{6}$

Also all Cichoń-characteristics are Blass-uniform. The Blass-uniform relational systems we use for these characteristics are (as in the Cichon's Maximum constructions) in some instances slightly different from the "canonical" ones. See, e.g., [BCM21, Ex. 2.16], [Mej19b, Ex. 2.10] and [GKS19, §1] for the definition of the Blass-uniform relational systems corresponding to the Cichoncharacteristics.

As in [GKMSa] we also look at relational systems $S=\langle S, S, \leq\rangle$ where $\leq$ is an upwards directed partial order on $S$. Here $\operatorname{cp}(S):=\mathfrak{b}(S)$ is the completeness of $S$, and $\operatorname{cof}(S):=\mathfrak{d}(S)$ is the cofinality of $S$. Recall that, whenever $S$ has no greatest element, $\operatorname{cp}(S) \leq \operatorname{cof}(S)$, and equality holds when the order is linear.

${ }^{6}$ It would be more natural to consider the relational system $\left\langle[\omega]^{\aleph_{0}},[\omega]^{\aleph_{0}}, R\right\rangle$ where $x R y$ iff either $x \supseteq^{*} y$ or $\omega \backslash x \supseteq^{*} y$, but $\mathbf{R}_{\mathrm{sp}}$ is more suitable in our proofs. It is not hard to see that both relational systems are Tukey-equivalent. 
The following is a very useful notion to calculate the value of cardinal characteristics (specially in forcing extensions).

Definition 3.2 (cf. [GKS19, §1]): Fix a directed partial order $S=\langle S, \leq\rangle$ and a relational system $\mathbf{R}=\langle X, Y, R\rangle$. Define the property:

Cone of bounds.

$\operatorname{COB}_{\mathbf{R}}(S)$ means: There is a family $\bar{y}=\left\{y_{i}: i \in S\right\} \subseteq Y$ such that

$$
\forall x \in X \exists i_{x} \in S \forall j \geq i_{x}\left(x R y_{j}\right) .
$$

When $L=\langle L, \leq\rangle$ is a linear order, we additionally define

\section{Linear cofinally unbounded.}

$\operatorname{LCU}_{\mathbf{R}}(L)$ means: There is a family $\bar{x}=\left\{x_{i}: i \in L\right\} \subseteq X$ such that

$$
\forall y \in Y \exists i \in L \forall j \geq i\left(\neg\left(x_{j} R y\right)\right) .
$$

In the following remarks we address very natural characterizations and consequences of these properties.

Remark 3.3 (Tukey connections and COB): Let $\bar{y}$ be a witness of $\operatorname{COB}_{\mathbf{R}}(S)$. By the definition of $\operatorname{COB}_{\mathbf{R}}(S)$ we have that the functions $f: X \rightarrow S$ and $g: S \rightarrow Y$, defined by $f(x):=i_{x}$ and $g(i):=y_{i}$, form a Tukey connection from $\mathbf{R}$ into $S$. So we conclude that

$$
\operatorname{COB}_{\mathbf{R}}(S) \text { holds iff } \mathbf{R} \leq_{\mathrm{T}} S,
$$

where $\leq_{\mathrm{T}}$ denotes the Tukey order.

Remark 3.4 (Duality and LCU): Let $\mathbf{R}=\langle X, Y, R\rangle$ be a relational system. The dual of $\mathbf{R}$ is the relational system $\mathbf{R}^{\perp}:=\left\langle Y, X, R^{\perp}\right\rangle$ where $u R^{\perp} v \Leftrightarrow \neg(v R u)$. It is clear that $\mathfrak{d}\left(\mathbf{R}^{\perp}\right)=\mathfrak{b}(\mathbf{R})$ and $\mathfrak{b}\left(\mathbf{R}^{\perp}\right)=\mathfrak{d}(\mathbf{R})$. Also, given a linear order $L$,

$$
\operatorname{LCU}_{\mathbf{R}}(L) \text { iff } \operatorname{COB}_{\mathbf{R}^{\perp}}(L) \text {. }
$$

Hence, by Remark 3.3,

$$
\operatorname{LCU}_{\mathbf{R}}(L) \text { iff } \mathbf{R}^{\perp} \leq_{\mathrm{T}} L .
$$

When $L$ has no greatest element, $L^{\perp}$ is Tukey-equivalent to $L$, so

$$
\operatorname{LCU}_{\mathbf{R}}(L) \text { iff } L \leq_{\mathrm{T}} \mathbf{R} .
$$

Although LCU is a particular case of COB, they are used with different roles in our applications, so it is more practical to use different notations.

As a direct consequence of these remarks: 
Lemma 3.5 (cf. [GKS19, §1]): Let $\mathbf{R}$ be a relational system, $S$ a directed partial order and let $L$ be a linear order without greatest element. Then:

(a) $\operatorname{COB}_{\mathbf{R}}(S)$ implies $\operatorname{cp}(S) \leq \mathfrak{b}(\mathbf{R})$ and $\mathfrak{d}(\mathbf{R}) \leq \operatorname{cof}(S)$.

(b) $\operatorname{LCU}_{\mathbf{R}}(L)$ implies $\mathfrak{b}(\mathbf{R}) \leq \operatorname{cp}(L)=\operatorname{cof}(L) \leq \mathfrak{d}(\mathbf{R})$.

In our applications we aim to force $\operatorname{COB}_{\mathbf{R}}(S)$ and $\operatorname{LCU}_{\mathbf{R}}(L)$ for a given relational system of the reals $\mathbf{R}$; this will help us compute the value of $\mathfrak{b}(\mathbf{R})$ and $\mathfrak{d}(\mathbf{R})$ in generic extensions. For this purpose, the following variation of Definition 3.2 is very practical.

Definition 3.6 ([GKMSa]): Let $\mathbf{R}=\langle X, Y, R\rangle$ be a relational system of the reals, $S=\left\langle S, \leq_{S}\right\rangle$ a directed partial order, $L=\left\langle L, \leq_{L}\right\rangle$ a linear order, and let $\mathbb{P}$ be a forcing notion. Define the following properties:

$\operatorname{COB}_{\mathbf{R}}(\mathbb{P}, S)$ : There is a family $\dot{\bar{y}}=\left\{\dot{y}_{i}: i \in S\right\}$ of $\mathbb{P}$-names of members of $Y^{V^{\mathbb{P}}}$ such that, for any $\mathbb{P}$-name $\dot{x}$ of a member of $X^{V^{\mathbb{P}}}$, there is some $i \in S$ such that

$$
\Vdash_{\mathbb{P}} \forall j \geq_{S} i\left(\dot{x} R \dot{y}_{j}\right) \text {. }
$$

$\operatorname{LCU}_{\mathbf{R}}(\mathbb{P}, L)$ : There is a family $\dot{\bar{x}}=\left\{\dot{x}_{i}: i \in L\right\}$ of $\mathbb{P}$-names of members of $X^{V^{\mathbb{P}}}$ such that, for any $\mathbb{P}$-name $\dot{y}$ of a member of $Y^{V^{\mathbb{P}}}$, there is some $i \in L$ such that

$$
\Vdash_{\mathbb{P}} \forall j \geq_{L} i\left(\neg\left(\dot{x}_{j} R \dot{y}\right)\right) .
$$

Remark 3.7: Concerning the properties $\operatorname{COB}_{\mathbf{R}}(\mathbb{P}, S)$ and $\operatorname{LCU}_{\mathbf{R}}(\mathbb{P}, L)$, the relational system $\mathbf{R}$ (i.e., both base sets as well as the relation) are interpreted in the generic extension (this is why we required these objects to be definable), while $S$ and $L$ are taken as sets in the ground model (not interpreted).

It is clear that $\operatorname{COB}_{\mathbf{R}}(\mathbb{P}, S)$ implies $\vdash_{\mathbb{P}} \operatorname{COB}_{\mathbf{R}}(S)$. Although the converse is not true in general, it holds in the cases we are interested in, when $\mathbb{P}$ is ccc and $\operatorname{cp}(S)$ is uncountable. More precisely, if $\operatorname{cp}(S)$ is uncountable and $\mathbb{P}$ is $\operatorname{cp}(S)$-cc then $\operatorname{COB}_{\mathbf{R}}(\mathbb{P}, S)$ is equivalent to $\Vdash_{\mathbb{P}} \operatorname{COB}_{\mathbf{R}}(S)$. Moreover, $\mathbb{P}$ forces

$$
\operatorname{cp}(S)^{V^{\mathbb{P}}}=\operatorname{cp}(S)^{V} \quad \text { and } \quad \operatorname{cof}(S)^{V^{\mathbb{P}}}=\operatorname{cof}(S)^{V},
$$

so, by Lemma 3.5, in the generic extension $\operatorname{COB}_{\mathbf{R}}(S)$ implies

$$
\operatorname{cp}(S)^{V} \leq \mathfrak{b}(\mathbf{R}) \quad \text { and } \quad \mathfrak{d}(\mathbf{R}) \leq \operatorname{cof}(S)^{V} .
$$

Likewise, $\operatorname{LCU}_{\mathbf{R}}(\mathbb{P}, L)$ implies $\Vdash_{\mathbb{P}} \operatorname{LCU}_{\mathbf{R}}(L)$, and the converse holds whenever $L$ has no greatest element, $\operatorname{cof}(L)$ is uncountable and $\mathbb{P}$ is $\operatorname{cof}(L)$-cc.

However, the restriction $" \operatorname{cp}(S)$ is uncountable and $\mathbb{P}$ is $\operatorname{cp}(S)$-cc" is not required for the following result. 
Lemma 3.8 ([GKMSa, Lemma 1.3]): Let $\mathbf{R}$ be a relational system of the reals, $S$ a directed partial order without greatest element, and let $\mathbb{P}$ be a forcing notion. If $\mu=\operatorname{cp}(S)^{V}$ and $\lambda=\operatorname{cof}(S)^{V}$, then:

(a) $\operatorname{COB}_{\mathbf{R}}(\mathbb{P}, S)$ implies $\Vdash_{\mathbb{P}} " \mu \leq \mathfrak{b}(\mathbf{R})$ and $\mathfrak{d}(\mathbf{R}) \leq|\lambda|$ ".

(b) If $L=S$ is a linear order, then $\mathrm{LCU}_{\mathbf{R}}(\mathbb{P}, L)$ implies

$$
\Vdash_{\mathbb{P}} \mathfrak{b}(\mathbf{R}) \leq|\lambda| \leq \lambda \leq \mathfrak{d}(\mathbf{R}) " .
$$

\section{Preserving splitting families with symmetric iterations}

4.A. The single forcing $\mathbb{G}_{\mathbf{B}}$. Using suitable 2-graphs, we define a poset which will be used as factor for the forcing adding the splitting families we aim to preserve.

Definition 4.1: Let $\mathbf{B}=\left\langle B, R_{0}, R_{1}\right\rangle$ be a suitable 2-graph. Define the forcing $\mathbb{G}_{\mathbf{B}}$ whose conditions are functions $p: F_{p} \times n_{p} \rightarrow\{0,1\}$ where $F_{p} \in[B]<\aleph_{0}$ and $n_{p}<\omega$ (also demand $F_{p}=\emptyset$ iff $n_{p}=\emptyset$ ). The order is defined by $q \leq p$ iff

(i) $p \subseteq q$,

(ii) for each $k \in\left[n_{p}, n_{q}\right)$, the map $F_{p} \rightarrow 2, a \mapsto q(a, k)$ respects $\mathbf{B}$, that is, if $e \in\{0,1\}, a, b \in F_{p}$, and $a R_{e} b$, then $\{q(a, k), q(b, k)\} \neq\{e\}$.

For $a \in B$ denote by $\dot{\eta}_{a}$ the name of the generic real added at $a$, that is, $\mathbb{G}_{\mathbf{B}}$ forces that, for any $k<\omega, \dot{\eta}_{a}(k)=e$ iff $p(a, k)=e$ for some $p$ in the generic set.

For $p \in \mathbb{G}_{\mathbf{B}}$ denote $\operatorname{supp} p:=F_{p}$.

Lemma 4.2: Let $\mathbf{B}=\left\langle B, R_{0}, R_{1}\right\rangle$ be a suitable 2-graph. Then:

(a) $\mathbb{G}_{\mathbf{B}}$ is $\sigma$-centered.

(b) For any $a \in B, \mathbb{G}_{\mathbf{B}}$ forces that $\dot{\eta}_{a}$ is Cohen over $V$.

(c) Any $p \in \mathbb{G}_{\mathbf{B}}$ forces that, for any $k \geq n_{p}$, the map $F_{p} \rightarrow 2$, a $\mapsto \dot{\eta}_{a}(k)$ respects $\mathbf{B}$, that is, if $e \in\{0,1\}, a, b \in F_{p}$ and $a R_{e} b$, then $\dot{\eta}_{a}(k)$ and $\dot{\eta}_{b}(k)$ cannot both be $e$ at the same time.

(d) Assume for $i \in\{1,2\}$ :

- $e \in\{0,1\}, p_{i} \in \mathbb{G}_{\mathbf{B}}, c_{i} \in F_{p_{i}}, c_{1} R_{e} c_{2}$,

- $\mathbb{Q}$ is a poset, $\mathbb{G}_{\mathbf{B}} \lessdot \mathbb{Q}$,

- $\dot{b}$ is a $\mathbb{Q}$-name of an infinite subset of $\omega$,

- $q_{i} \leq p_{i}$ in $\mathbb{Q}$ and $q_{i} \Vdash_{\mathbb{Q}} \dot{\eta}_{c_{i}} \mid \dot{b} \equiv e$.

Then $q_{1}$ and $q_{2}$ are incompatible.

(e) If $f: B \rightarrow B$ is a B-automorphism, then $\hat{f}: \mathbb{G}_{\mathbf{B}} \rightarrow \mathbb{G}_{\mathbf{B}}$ defined by $\hat{f}(p)(\alpha, n)=p\left(f^{-1}(\alpha), n\right)$ (where $F_{\hat{f}(p)}:=f\left[F_{p}\right]$ ) is a p.o.-automorphism. 
Proof. To see (a), first note that since $|B \times \omega|=\aleph_{1}$, by Engelking-Karłowicz [EK65] there is a countable set $H \subseteq 2^{B \times \omega}$ such that any finite partial function from $B \times \omega$ into 2 can be extended by some member of $H$. For $h \in H$ and $n<\omega$, let $C_{h, n}:=\left\{p \in \mathbb{G}_{\mathbf{B}}: p \subseteq h\right.$ and $\left.n_{p}=n\right\}$. It is clear that $C_{h, n}$ is centered and

$$
\mathbb{G}_{\mathbf{B}}=\bigcup_{h \in H} \bigcup_{n<\omega} C_{h, n},
$$

so $\mathbb{G}_{\mathbf{B}}$ is $\sigma$-centered.

(b): Consider Cohen forcing $\mathbb{C}:=2^{<\omega}$ ordered by end-extension. For $a \in B$ define $\operatorname{pr}_{a}: \mathbb{G}_{\mathbf{B}} \rightarrow \mathbb{C}$ such that, for any $p \in \mathbb{G}_{\mathbf{B}}, \operatorname{pr}_{a}(p):=\left\langle p(a, k): k<n_{p}\right\rangle$ if $a \in \operatorname{supp} p$, or $\operatorname{pr}_{a}(p)$ is the empty sequence otherwise. It is enough to show that $\mathrm{pr}_{a}$ is a forcing projection, that is,

(i) for any $p, q \in \mathbb{G}_{\mathbf{B}}$, if $q \leq p$ then $\operatorname{pr}_{a}(q) \supseteq \operatorname{pr}_{a}(p)$,

(ii) for any $p \in \mathbb{G}_{\mathbf{B}}$ and $s \in \mathbb{C}$, if $s \supseteq \operatorname{pr}_{a}(p)$ then there is some $q \leq p$ in $\mathbb{G}_{B}$ such that $\operatorname{pr}_{a}(q) \supseteq s\left(\right.$ even $\left.\operatorname{pr}_{a}(q)=s\right)$,

(iii) $\operatorname{pr}_{a}\left[\mathbb{C}_{\mathbf{B}}\right]$ is dense in $\mathbb{C}$ (even $\operatorname{pr}_{a}$ is onto).

Property (i) is easy, (ii) follows by Definition 2.1(v), and (iii) follows by (ii) and the fact that $\operatorname{pr}_{a}(\emptyset)=\langle\rangle$.

(c): By the definition of the order of $\mathbb{G}_{\mathbf{B}}$.

(d): Assume $q \in \mathbb{Q}$ is stronger than $q_{1}$ and $q_{2}$, so

$$
q \Vdash "\left\{k<\omega: \dot{\eta}_{c_{1}}(k)=\dot{\eta}_{c_{2}}(k)=e\right\} \text { is infinite". }
$$

Hence, there is some $p \in \mathbb{G}_{\mathbf{B}}$ stronger than $p_{1}$ and $p_{2}$ forcing the same, but this contradicts (c) because $c_{1}, c_{2} \in F_{p}$ and $c_{1} R_{e} c_{2}$.

(e) is straightforward.

Remark 4.3: The obvious restriction of $\mathbb{G}_{\mathbf{B}}$ to, say, the first two coordinates, is not a projection, and $\mathbb{G}_{\mathbf{B}}$ is not a FS iteration of length $\omega_{1}$ in any natural way. Assume, e.g., we restrict to $\{0,1\} \subseteq B=\omega_{1}$, and $\mathbf{B}$ contains an $e$-colored edge from node $e$ to node 2 for $e \in\{0,1\}$. Start with a condition $p:\{0,1,2\} \times n \rightarrow 2$ (for, e.g., $n=1$ ), restrict it to $p^{-}=p\left\lceil\{0,1\}\right.$ and extend it to $p^{\prime} \in \mathbb{G}_{\mathbf{B}\lceil\{0,1\}}$ by setting $p^{\prime}(e, n)=e$ for $e \in\{0,1\}$. Then there is no $q \in \mathbb{G}_{\mathbf{B}}, q \leq p$, compatible with $p^{\prime}$.

We will use FS iterations where the first step is given by a FS product of posets of the form $\mathbb{G}_{\mathbf{B}}$ as above. It is clear that, if $\mathbf{B}$ is a $\mathrm{S} 2 \mathrm{G}$ in the ground model, then it is still a $\mathrm{S} 2 \mathrm{G}$ in any extension preserving $\omega_{1}$. On the other hand, 
constructing $\mathbb{G}_{\mathbf{B}}$ from $\mathbf{B}$ is absolute for transitive models of ZFC, so any finite support product of posets of the form $\mathbb{G}_{\mathbf{B}}$ is forcing equivalent to their finite support iteration (as long as the sequence of 2-graphs lives in the ground model).

4.B. Suitable iterations, nice names and automorphisms. We now introduce some notions associated with these iterations, relevant for the preservation of splitting families.

From this point on, products of ordinals (such as $\omega_{1} \pi$ ) should be interpreted as ordinal products.

Definition 4.4: A suitable iteration is defined by the following objects:

(I) A cardinal $\pi_{0}>0$.

(II) For each $\delta<\pi_{0}$, a $\mathrm{S} 2 \mathrm{G} \mathbf{B}_{\delta}=\left\langle B_{\delta}, R_{\delta, 0}, R_{\delta, 1}\right\rangle$ with $B_{\delta}:=\left[\omega_{1} \delta, \omega_{1}(\delta+1)\right)$,

(III) an ordinal $\pi \geq \pi_{1}:=\omega_{1} \pi_{0}$,

(IV) a FS ccc iteration $\mathbb{P}$ of length $1+\left(\pi-\pi_{1}\right)$ where the first iterand is the FS product of the $\mathbb{G}_{\mathbf{B}_{\delta}}$ for $\delta<\pi_{0}$, called $\mathbb{P}_{\pi_{1}}$, and the following iterands are indexed by $\xi \in \pi \backslash \pi_{1}$ and are ccc posets called $\dot{\mathbb{Q}}_{\xi}$.

As usual, we denote with $\mathbb{P}_{\xi}$ the result of the iteration up to $\xi$ (for $\pi_{1} \leq \xi \leq \pi$ ), and use $\mathbb{P}$ to denote either $\mathbb{P}_{\pi}$ or the whole iteration (or its definition). See Figure 5 for an illustration.

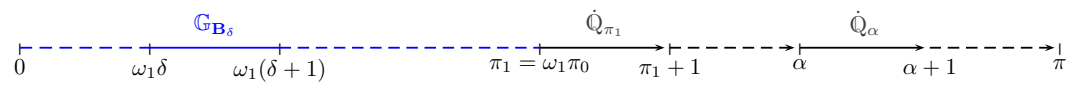

Figure 5. A suitable iteration. $\pi_{1}=\omega_{1} \pi_{0}$ is partitioned into $\pi_{0}$-many intervals of length $\omega_{1}$, and $B_{\delta}:=\left[\omega_{1} \delta, \omega(\delta+1)\right)$, the set of vertices of the graph $\mathbf{B}_{\delta}$, is the $\delta$-th interval of this partition. A suitable iteration is a FS product of the $\mathbb{G}_{\mathbf{B}_{\delta}}$ for $\delta<\pi_{0}$, followed by a FS iteration of ccc posets. The iterands of the FS iteration that follow are indexed by $\alpha \in\left[\pi_{1}, \pi\right)$.

Remark 4.5: Note that we could also view $\mathbb{P}_{\pi_{1}}$ as (the result of) a FS-iteration of length $\pi_{0}$ (instead of length 1 , as we do in the definition). Then we would get an iteration $\mathbb{P}$ of $\pi_{0}+\left(\pi-\pi_{1}\right)$. However, $\mathbb{P}_{\pi_{1}}$ is not a FS iteration of length $\pi_{1}$, at least not with natural iterands; see Remark 4.3.

Let us mention some notation: 
Notation 4.6: (1) A real-number-poset is a poset whose universe is a subset of the set of real numbers. For simplicity, we identify the "set of real numbers" with the power set of $\omega$.

(2) For notational simplicity we will often identify $\mathbb{P}_{\zeta+1}$ (a set of partial functions) with $\mathbb{P}_{\zeta} * \dot{\mathbb{Q}}_{\zeta}$ (a set of pairs $(p, q)$ with $p \in \mathbb{P}_{\zeta}$ and $p \Vdash q \in \dot{\mathbb{Q}}_{\zeta}$ ).

(3) Similarly, we will not distinguish between sequences of names and names of sequences.

We now define the "support" $\operatorname{supp}(p) \subseteq \pi$ of a condition $p$ (as opposed to the domain $\operatorname{dom}(p)$, which is, as we are dealing with a FS iteration, a finite subset of the index set $\left.\{0\} \cup\left(\pi \backslash \pi_{1}\right)\right)$. We will also define the "history" $H$ of a name and of a condition:

Definition 4.7: Let $\mathbb{P}$ be a suitable iteration.

(1) For $p \in \mathbb{P}_{\pi_{1}}$ set

$$
\operatorname{supp}(p):=\bigcup_{\delta \in \operatorname{dom} p} \operatorname{supp}(p(\delta)) \subseteq \pi_{1}
$$

For $p \in \mathbb{P}$, set

$$
\operatorname{supp} p:=\operatorname{supp}(p(0)) \cup(\operatorname{dom}(p) \backslash\{0\})
$$

(or just $\operatorname{dom}(p)$, if $0 \notin \operatorname{dom}(p))^{7}$

(2) For $p \in \mathbb{P}$ and a $\mathbb{P}$-name $\tau$, we define $H(p) \subseteq \pi$ and $H(\tau) \subseteq \pi$ as follows:

(i) For $p \in \mathbb{P}_{\pi_{1}}, H(p):=\operatorname{supp} p$.

For $\xi \geq \pi_{1}$ we define $H$ by recursion on $\xi$ for $p \in \mathbb{P}_{\xi}$ and for a $\mathbb{P}_{\xi}$-name $\tau$. (We assume that $H(r)$ has been defined for all $r \in \mathbb{P}_{\zeta}$ for $\pi_{1} \leq \zeta<\xi$ and

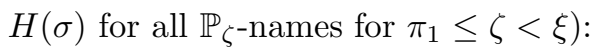

(ii) For $\xi=\zeta+1$ and $p \in \mathbb{P}_{\zeta+1}$,

$$
H(p):= \begin{cases}H(p\lceil\zeta) & \text { if } \zeta \notin \operatorname{supp} p \\ H(p\lceil\zeta) \cup\{\zeta\} \cup H(p(\zeta)) & \text { if } \zeta \in \operatorname{supp} p .\end{cases}
$$

(Here, $H(p(\zeta))$ is defined because $p(\zeta)$ is a $\mathbb{P}_{\zeta}$-name.)

\footnotetext{
7 Recall that according to our indexing, $\operatorname{dom}(p)$ is a finite subset of $\{0\} \cup\left(\pi \backslash \pi_{1}\right)$ (where we interpret a FS condition $p$ as a partial function from the index $\pi_{0}$ with finite domain $\operatorname{dom}(p))$. Recall that $q:=p(0) \in \mathbb{P}_{\pi_{1}}$, which is the FS product of $\mathbb{G}_{\mathbf{B}_{\delta}}$ for $\delta<\pi_{0}$. So $q$ has a finite domain $\operatorname{dom}(q) \subseteq \pi_{0}$, and if $\delta \in \operatorname{dom}(q)$, then $q(\delta) \in \mathbb{G}_{\mathbf{B}_{\delta}}$, so $X_{\delta}=\operatorname{supp}(q(\delta))$ (in the sense of the forcing $\mathbb{G}_{\mathbf{B}_{\delta}}$ ) is a finite subset of $\left[\omega_{1} \delta, \omega_{1}(\delta+1)\right.$ ). According to our definition, $\operatorname{supp}(q)=\bigcup_{\delta \in \operatorname{dom}(q)} X_{\delta}$.
} 
(iii) When $\xi>\pi_{1}$ is limit and $p \in \mathbb{P}_{\xi}$, then $H(p)$ has already been defined (because $p \in \mathbb{P}_{\zeta}$ for some $\zeta<\xi$ ).

(iv) For any $\mathbb{P}_{\xi}$-name $\tau$ define (by $\in$-recursion on $\tau$ )

$$
H(\tau):=\bigcup\{H(\sigma) \cup H(p):(\sigma, p) \in \tau\} .
$$

Note that $H(\check{x})=\emptyset$ for any standard name $\check{x}^{8}$

Remark: $H$ is not a "robust" notion: $\Vdash \tau=\tau^{\prime}$ does not imply $H(\tau)=H\left(\tau^{\prime}\right)$. Still, it is a very natural and useful notion, which has appeared (in slightly different contexts) many times in forcing theory: If $\tau$ is a $\mathbb{P}_{\pi}$-name, then $H(\tau) \subseteq \pi$ is the set of coordinates the name $\tau$ "depends on", more concretely, $\tau$ can be calculated (by a function defined in $V$ ) from the sequence of generic objects at the indices in $H(\tau)$.

In the case of FS iterations where all iterands are real-number-posets (as in [She00, GKS19]), $H(p)$ is countable for $p$ in a dense set; and "hereditarily nice names" for reals will also have countable history. In this paper we have to use hereditarily $<\lambda$-names (even for nice names of reals); the reason is indicated in Remark 4.16.

Let us fix some notation regarding the well-known "nice names":

Definition 4.8: Let $A$ and $B$ be subsets of $\mathbb{P}$.

(1) A $\mathbb{P}$-name $\dot{r}$ is a nice name for a subset of $\omega$, determined by $A$, if $\dot{r}$ has the form $\bigcup_{n \in \omega}\left\{(\check{n}, q): q \in A_{n}\right\}$, where each $A_{n}$ is a (possibly empty) antichain in $\mathbb{P}$, and $A=\bigcup_{n \in \omega} A_{n}$.

(2) Analogously, $\dot{Q}$ is a nice name for a real-number-poset of size $<\lambda$, determined by $B$, if there is a $\mu<\lambda$ such that $\dot{Q}$ is a sequence $\left\langle\dot{r}_{i}\right\rangle_{i \in \mu}$ of nice names for subsets of $\omega$ determined by $A_{i}$, together with a sequence $\left\langle\dot{x}_{i, j}\right\rangle_{i, j \in \mu}$ of nice names for elements in $\{0,1\}$ depending on an antichain $A_{i, j}^{\prime}$ (where $\dot{x}_{i, j}=1$ codes $\left.r_{i} \leq_{Q} r_{j}\right),{ }^{9}$ and $B=\bigcup_{i \in \mu} A_{i} \cup \bigcup_{i, j \in \mu} A_{i, j}^{\prime}$.

8 A standard name $\check{x}=\{(\check{y}, \mathbb{1}): y \in x\}$ (for $x \in V$ ) hereditarily only uses the weakest condition $\mathbb{1}$, which in our case (an iteration) is the empty partial function; accordingly $H(\check{x})=\emptyset$. If the reader prefers a different formal definition of FS iteration, then they should modify the definition of $H$ to make sure that $H(\check{x})=\emptyset$.

9 A nice name $\dot{x}$ of a member of $\{0,1\}$ depending on an antichain $C \subseteq \mathbb{P}$ (allowed to be empty) has the form $\dot{x}=\{(\check{0}, p): p \in C\}$. Note that $p \Vdash \dot{x}=1$ for all $p \in C$, and $q \Vdash \dot{x}=0$ for any $q \in \mathbb{P}$ incompatible with all the members of $C$. Moreover, $H(\dot{x})=\bigcup_{p \in C} H(p)$. 
So in this case

$$
H(\dot{r})=\bigcup_{p \in A} H(p), \quad \text { and } \quad H(\dot{Q})=\bigcup_{p \in B} H(p) .
$$

It is well known that every name of a subset of $\omega$ has an equivalent nice name. Moreover, as we can choose the conditions of the antichains in any given dense set, we get the following:

FACT 4.10 (As $\mathbb{P}$ is ccc): Let $D \subseteq \mathbb{P}$ be dense and let $\lambda$ be a cardinal with uncountable cofinality.

(a) For any $\mathbb{P}$-name of a real there is an equivalent nice name determined by $A \subseteq D$ with $|A| \leq \aleph_{0}$.

(b) For any name of a poset of size $<\lambda$ consisting of reals, there is an equivalent nice name determined by a set $B \subseteq D$ with $|B|<\lambda$.

Every automorphism of B induces an automorphism of $\mathbb{G}_{\mathbf{B}}$; see Lemma 4.2(e). Therefore, a $\pi_{0}$-sequence $h$ of such automorphisms induces an automorphism of the (FS) product $\mathbb{P}_{\pi_{1}}$. Such an automorphism can sometimes be naturally extended to the whole iteration $\mathbb{P}$ (which will allow isomorphism-of-names arguments and subsequently show $\left.\mathrm{LCU}_{\mathrm{sp}}\right)$.

What do we mean by "naturally extend"? Recall that, whenever $f: P \rightarrow P$ is an automorphism on some poset $P$, and $\tau$ is a $P$-name, $f$ sends $\tau$ to the $P$-name

$$
f^{*}(\tau):=\left\{\left(f^{*}(\sigma), f(p)\right):(\sigma, p) \in \tau\right\} .
$$

Also, $\left(f^{-1}\right)^{*}\left(f^{*}(\tau)\right)=\tau$; and $p \Vdash \varphi(\tau)$ iff $f(p) \Vdash \varphi\left(f^{*}(\tau)\right)$ whenever $p \in P$ and $\varphi(x)$ is a formula. If $\dot{Q}$ is a $P$-name and $P \Vdash f^{*}(\dot{Q})=\dot{Q}$, then we can certainly extend $f$ to $P * \dot{Q}$. We say that $\mathbb{P}$ is $h$-symmetric, if this is the case in all steps of the iteration:

Definition 4.11: Let $\mathbb{P}$ be a suitable iteration.

(1) A bijection $h: \pi_{1} \rightarrow \pi_{1}$ is a 2G-automorphism if, for each $\delta<\pi_{0}, h\left\lceil B_{\delta}\right.$ is an automorphism of $\mathbf{B}_{\delta}$.

(2) Such an $h$ defines an automorphism $\hat{h}_{\pi_{1}}$ of $\mathbb{P}_{\pi_{1}} \rightarrow \mathbb{P}_{\pi_{1}}$, by

$$
\hat{h}_{\pi_{1}}(p):=\left\langle\hat{f}_{\delta}(p(\delta)): \delta \in \operatorname{dom} p\right\rangle
$$

where $f_{\delta}:=h \uparrow\left[\omega_{1} \delta, \omega_{1}(\delta+1)\right)$ is the automorphism of $\mathbf{B}_{\delta}$ induced by $h$, and $\hat{f}_{\delta}$ is defined as in Lemma $4.2(\mathrm{e})$. 
(3) We say $\mathbb{P}$ is $h$-symmetric if the following inductive construction defines $\hat{h}_{\xi}: \mathbb{P}_{\xi} \rightarrow \mathbb{P}_{\xi}$ for all $\pi_{1} \leq \xi \leq \pi:$

(i) For $\xi=\zeta+1$, we require that $\Vdash_{\mathbb{P}_{\zeta}} \hat{h}_{\zeta}^{*}\left(\dot{\mathbb{Q}}_{\zeta}\right)=\dot{\mathbb{Q}}_{\zeta}$. (Otherwise the construction fails.) We then define $\hat{h}_{\zeta+1}: \mathbb{P}_{\zeta+1} \rightarrow \mathbb{P}_{\zeta+1}$ by

$$
\hat{h}_{\zeta+1}\left(p\lceil\zeta, p(\zeta))=\left(\hat{h}_{\zeta}\left(p\lceil\zeta), \hat{h}_{\zeta}^{*}(p(\zeta))\right)\right.\right.
$$

(ii) For $\xi>\pi_{1}$ limit, set $\hat{h}_{\xi}:=\bigcup_{\zeta<\xi} \hat{h}_{\zeta}$.

In this case set $\hat{h}:=\hat{h}_{\pi}$, which is an automorphism of $\mathbb{P}$.

(4) For any $\delta<\pi_{0}$ and any pair $(a, b) \in B_{\delta}$, fix a 2G-automorphism $h_{a, b}^{\delta}$ such that $h_{a, b}^{\delta}(a)=b$ and $h_{a, b}^{\delta} \backslash \mathbf{B}_{\zeta}$ is the identity for any $\zeta \neq \delta$. We can pick such $h_{a, b}^{\delta}$ by Definition 2.1(vi).

(5) Let $\mathcal{H}^{*}$ be the group generated by the $h_{a, b}^{\delta}$ above. So $\left|\mathcal{H}^{*}\right|=\max \left\{\pi_{0}, \aleph_{1}\right\}$. Note also that for all $h \in \mathcal{H}^{*}$ and $\delta \in \pi_{0}$ we have $h\left[B_{\delta}\right]=B_{\delta}$, and that $\operatorname{supp}(h):=\bigcup\left\{B_{\delta}: h\left\lceil B_{\delta} \neq \operatorname{id}_{B_{\delta}}, \delta<\pi_{0}\right\}\right.$ has size $\leq \aleph_{1}$.

(6) We say that $\mathbb{P}$ is symmetric if $\mathbb{P}$ is $h$-symmetric for every $h \in \mathcal{H}^{*}$.

In isomorphism-of-names arguments it is relevant to know when a condition or a name remains unchanged after applying an automorphism $\hat{h}$. The following states a sufficient condition:

Lemma 4.12: Assume that $\mathbb{P}$ is $h$-symmetric and $\pi_{1} \leq \xi \leq \pi$.

(a) If $p \in \mathbb{P}_{\xi}$ and $h \uparrow\left(H(p) \cap \pi_{1}\right)$ is the identity, then $\hat{h}_{\xi}(p)=p$.

(b) If $\tau$ is a $\mathbb{P}_{\xi}$-name and $h \uparrow\left(H(\tau) \cap \pi_{1}\right)$ is the identity, then $\hat{h}_{\xi}^{*}(\tau)=\tau$.

(c) Let $g:=h^{-1}$. Then $\mathbb{P}$ is $g$-symmetric and $\hat{g}_{\xi}=\hat{h}_{\xi}^{-1}$.

Proof. We show the three statements by induction on $\xi$.

For (a), we use a case distinction: Assume $\xi=\pi_{1}$. If $p \in \mathbb{P}_{\pi_{1}}$ then $H(p)=\operatorname{supp} p$, and whenever $h$ is the identity on $\operatorname{supp} p$, it is clear that $\hat{h}_{\pi_{1}}(p)=p$. The limit step is also immediate (there are no new conditions, and for names use $\in$-induction).

For the successor step $\xi=\zeta+1$, assume $p \in \mathbb{P}_{\zeta+1}$ and that $h$ is the identity on $H(p) \cap \pi_{1}$. If $\zeta \notin \operatorname{supp} p$, then we have $p \in \mathbb{P}_{\zeta}$, so $\hat{h}_{\zeta+1}(p)=\hat{h}_{\zeta}(p)=p$ by the induction hypothesis. So assume $\zeta \in \operatorname{supp} p$. Then

$$
H(p)=H(p\lceil\zeta) \cup\{\zeta\} \cup H(p(\zeta))
$$

so by induction hypothesis $\hat{h}_{\zeta}\left(p\lceil\zeta)=p\left\lceil\zeta\right.\right.$ and $\hat{h}_{\zeta}^{*}(p(\zeta))=p(\zeta)$, thus $\hat{h}_{\zeta+1}(p)=p$. 
We now show (b) by $\in$-induction on $\tau$. If $(\sigma, p) \in \tau$ then $H(\sigma) \cup H(p) \subseteq H(\tau)$, so by induction hypothesis and $(\mathrm{a}), \hat{h}_{\xi}^{*}(\sigma)=\sigma$ and $\hat{h}_{\xi}(p)=p$. Hence

$$
\hat{h}_{\xi}^{*}(\tau)=\left\{\left(\hat{h}_{\xi}^{*}(\sigma), \hat{h}_{\xi}(p)\right):(\sigma, p) \in \tau\right\}=\{(\sigma, p):(\sigma, p) \in \tau\}=\tau .
$$

For (c), the steps $\xi=\pi_{1}$ and $\xi>\pi_{1}$ limit are easy, so we deal with the successor step $\xi=\zeta+1$. So assume that $\hat{g}_{\zeta}$ is defined and $\hat{g}_{\zeta}=\hat{h}_{\zeta}^{-1}$. Since $\hat{h}_{\xi}$ is defined, $\Vdash_{\mathbb{P}_{\zeta}} \hat{h}_{\zeta}^{*}\left(\dot{\mathbb{Q}}_{\zeta}\right)=\dot{\mathbb{Q}}_{\zeta}$, which implies $\Vdash_{\mathbb{P}_{\zeta}} \hat{g}_{\zeta}^{*}\left(\dot{\mathbb{Q}}_{\zeta}\right)=\dot{\mathbb{Q}}_{\zeta}$, so $\hat{g}_{\zeta+1}$ is defined; and for any $p \in \mathbb{P}_{\xi}$,

$$
\hat{g}_{\xi}\left(\hat{h}_{\xi}(p)\right)=\left(\hat{g}_{\zeta}\left(\hat{h}_{\zeta}(p\lceil\zeta)), \hat{g}_{\zeta}^{*}\left(\hat{h}_{\zeta}^{*}(p(\zeta))\right)\right)=p,\right.
$$

so $\hat{g}_{\xi}=\hat{h}_{\xi}^{-1}$.

4.C. A digression: Self-indexed products. How to construct a symmetric iteration $\mathbb{P}$ ? We have to make sure that at each step $\zeta$ the iterand $\dot{\mathbb{Q}}_{\zeta}$ is invariant under $\hat{h}$ for all $h \in \mathcal{H}^{*}$. One case that will be useful: $\dot{\mathbb{Q}}_{\zeta}$ is a (ccc) FS product such that whenever $\dot{Q}$ is one of the factors, then $\hat{h}^{*}(\dot{Q})$ is also one.

But there is a technical difficulty here: We need $\Vdash_{\mathbb{P}_{\zeta}} \hat{h}^{*}\left(\dot{\mathbb{Q}}_{\zeta}\right)=\dot{\mathbb{Q}}_{\zeta}$ (i.e., really equality, not just isomorphism; as we want to get an actual automorphism of $\mathbb{P}_{\zeta+1}$ ). This is not possible if we "naively" index the product with an ordinal. For example, assume $\dot{Q}_{0}, \dot{Q}_{1}$ are such that

$$
\Vdash_{\mathbb{P}_{\zeta}} \hat{h}^{*}\left(\dot{Q}_{i}\right)=\dot{Q}_{1-i} \neq \dot{Q}_{i} .
$$

Then $\dot{Q}_{0} \times \dot{Q}_{1}$ (the product with index set $\{0,1\}$ ) is not a valid choice for $\mathbb{Q}_{\zeta}$, as

$$
\Vdash_{\mathbb{P}_{\zeta}} \hat{h}^{*}\left(\dot{Q}_{0} \times \dot{Q}_{1}\right)=\dot{Q}_{1} \times \dot{Q}_{0} \neq \dot{Q}_{0} \times \dot{Q}_{1} .
$$

So instead, we define (in the extension) the FS product $\prod \mathcal{F}$ of a set $\mathcal{F}$ of posets as the set of all finite partial functions $p$ from $\mathcal{F}$ into $\bigcup \mathcal{F}$ satisfying $p(Q) \in Q$ for all $Q \in \operatorname{dom}(p)$. We call this object the self-indexed product of the set $\mathcal{F}$.

In our framework, we start with a ground model set $\Xi_{\zeta}$ of $\mathbb{P}_{\zeta}$-names of posets. In the $\mathbb{P}_{\zeta}$-extension we let $\mathcal{F}$ be the set of evaluations of the names in $\Xi_{\zeta}$, and let $\dot{\mathbb{Q}}_{\zeta}$ be the self-indexed product of $\mathcal{F}$. 
Assume that all automorphisms from $\mathcal{H}^{*}$ can be extended up to $\zeta{ }^{10}$ We assume that $\Xi_{\zeta}$ is closed under each $h \in \mathcal{H}^{*}$, i.e., $\dot{Q} \in \Xi_{\zeta}$ implies $\hat{h}_{\zeta}^{*}(\dot{Q}) \in \Xi_{\zeta}$. So as $\Xi_{\zeta}$ is also closed under the inverse of $h$, by Lemma 4.12(c) we even get

$$
\hat{h}_{\zeta}^{*}\left[\Xi_{\zeta}\right]=\Xi_{\zeta} .
$$

So in particular $\hat{h}_{\zeta}^{*}\left[\Xi_{\zeta}\right]$ and $\Xi_{\zeta}$ evaluate to the same set and thus yield the same self-indexed product, i.e., $\Vdash \hat{h}_{\zeta}^{*}\left(\dot{\mathbb{Q}}_{\zeta}\right)=\dot{\mathbb{Q}}_{\zeta}$.

We record this fact for later reference:

FACT 4.13: Assume that $\dot{\mathbb{Q}}_{\zeta}$ is a "self-indexed" product of $\Xi_{\zeta}$, and that $\dot{Q} \in \Xi_{\zeta}$ implies $\hat{h}_{\zeta}^{*}(\dot{Q}) \in \Xi_{\zeta}$ for all $h \in \mathcal{H}^{*}$. Then $\mathbb{P}$ forces $\hat{h}_{\zeta}^{*}\left(\dot{\mathbb{Q}}_{\zeta}\right)=\dot{\mathbb{Q}}_{\zeta}$, so we can extend each $h \in \mathcal{H}^{*}$ to $\mathbb{P}_{\zeta} * \dot{\mathbb{Q}}_{\zeta}$.

We additionally assume that each factor is (forced to be) a real-number-poset. Assume that $(p, q) \in \mathbb{P}_{\zeta} * \dot{\mathbb{Q}}_{\zeta}$. We can densely assume that $p$ decides the finite domain of $q$, more specifically, the ${ }^{11}$ finite set $y \subseteq \Xi_{\zeta}$ such that $p$ forces that $\operatorname{dom}(q)$ is (the set of evaluations of) $y$. Also, for each $\dot{Q} \in y$, we can assume that $q(\dot{Q})$ is a nice name for a real, determined by some $A_{\dot{Q}}$. As usual, we can use a given dense set $D \subseteq \mathbb{P}_{\zeta}$ instead of $\mathbb{P}_{\zeta}$.

For later reference:

FACT 4.14: Assume that $\dot{\mathbb{Q}}_{\zeta}$ is a "self-indexed" product of $\Xi_{\zeta}$ as described above, that each factor is forced to be a set of reals, and that $D \subseteq \mathbb{P}_{\zeta}$ is dense. If $(p, q) \in \mathbb{P}_{\zeta} * \dot{\mathbb{Q}}_{\zeta}$, then there is a $\left(p^{\prime}, q^{\prime}\right) \leq(p, q)$ such that $p^{\prime} \in D$ decides the (finite) $\operatorname{dom}\left(q^{\prime}\right)$, and each $q^{\prime}(\dot{Q})$ is a nice name determined by some $A_{\dot{Q}} \subseteq D$. So in particular

$$
H\left(\left(p^{\prime}, q^{\prime}\right)\right)=H\left(p^{\prime}\right) \cup\{\zeta\} \cup \bigcup_{\dot{Q} \in \operatorname{dom}\left(q^{\prime}\right)}\left(H(\dot{Q}) \cup \bigcup_{r \in A_{\dot{Q}}} H(r)\right) .
$$

Remark 4.16: This is the reason hereditarily countable nice names are not sufficient in our setting to describe reals: Even the index $\dot{Q}$ in such a product $\dot{\mathbb{Q}}_{\zeta}$ is too complicated. However, as all the self-indexed products $\dot{\mathbb{Q}}_{\zeta}$ we use will have factors $\dot{Q}$ of size $<\lambda$, it turns out we can restrict ourselves to hereditarily $<\lambda$-names (this will be the dense set $\mathbb{P}_{\zeta}^{*}$ of Definition 5.3).

10 For this part all the properties of $\mathcal{H}^{*}$ are not required; it is just enough that $h \in \mathcal{H}^{*}$ implies $h^{-1} \in \mathcal{H}^{*}$.

11 Or rather: $a$ finite set, as different names in $\Xi_{\zeta}$ might evaluate to the same object, i.e., index. 
4.D. Symmetric SMALl History ITERATIONS PRESERVE SPLITTING FAMILIES. We are finally ready to prove the central fact about preservation of splitting families.

Definition 4.17: Let $\lambda$ be an uncountable cardinal.

(1) A condition $q \in \mathbb{P}$ is $\lambda$-small, if $\left|\left\{\delta<\pi_{0}: H(q) \cap B_{\delta} \neq \emptyset\right\}\right|<\lambda$.

(2) A suitable iteration $\mathbb{P}$ has $\lambda$-small history if, for any $p \in \mathbb{P}$, there is a $\lambda$-small $q \leq p$.

So in particular if $\mathbb{P}$ has $\lambda$-small history and $\dot{x}$ is a name of a subset of $\omega$, then there is an equivalent nice name $\dot{b}$ which only uses $\lambda$-small conditions; and if $\mu \geq \lambda$ has uncountable cofinality, then

$$
\left|\left\{\delta<\pi_{0}: H(\dot{b}) \cap B_{\delta} \neq \emptyset\right\}\right|<\mu .
$$

Theorem 4.19: Let $\mathbb{P}$ be a symmetric suitable iteration with $\lambda$-small history. Assume $\aleph_{1} \leq \lambda \leq \mu \leq \pi_{0}$ are cardinals with $\mu$ regular. Then $\operatorname{LCU}_{\mathbf{R}_{\mathrm{sp}}}\left(\mathbb{P}_{\pi}, \mu\right)$ holds, and it is witnessed by $\left\{\dot{\eta}_{\omega_{1} \delta}: \delta<\mu\right\}$.

Proof. Towards a contradiction, assume that there are $p \in \mathbb{P}$ and a $\mathbb{P}$-name $\dot{b}$ of an infinite subset of $\omega$ such that

$$
p \Vdash \mid\left\{\delta<\mu: \dot{\eta}_{\omega_{1} \delta} \mid \dot{b} \text { is eventually constant }\right\} \mid=\mu .
$$

Find $F \in[\mu]^{\mu}, n_{0}<\omega$ and $e \in\{0,1\}$ such that, for any $\delta \in F$, there is some $p_{\delta} \leq p$ in $\mathbb{P}$ such that $\omega_{1} \delta \in \operatorname{supp}\left(p_{\delta}\right)$ and $p_{\delta} \Vdash \dot{\eta}_{\omega_{1} \delta}\left\lceil\left(\dot{b} \backslash n_{0}\right) \equiv e\right.$.

We can assume that $\dot{b}$ is a nice name, more particularly that (4.18) holds, and we can also assume that $p$ is $\lambda$-small. So there is some $\delta_{0} \in F$ such that $B_{\delta_{0}} \cap(H(p) \cup H(\dot{b}))=\emptyset$.

Put $a:=\omega_{1} \delta_{0} \in B_{\delta_{0}}$. By (2.2), $a$ is contained in an uncountable $R_{\delta_{0}, e^{-}}$ complete $U \subseteq B_{\delta_{0}}$. Recall that by the definition of "symmetric", there is for each $c \in U$ a 2G-automorphism $h^{c} \in \mathcal{H}^{*}$ such that $h^{c}(a)=c$ and such that $h^{c} \uparrow \mathbf{B}_{\delta}$ is the identity for all $\delta \neq \delta_{0}$. Hence, by Lemma $4.12, \hat{h}_{\pi}^{c}(p)=p$ and $\left(\hat{h}_{\pi}^{c}\right)^{*}(\dot{b})=\dot{b}$, therefore $p_{c}^{\prime}:=\hat{h}_{\pi}^{c}\left(p_{\delta_{0}}\right) \leq p$ and, since $\left(\hat{h}_{\pi}^{c}\right)^{*}\left(\dot{\eta}_{a}\right)=\dot{\eta}_{c}$,

$$
p_{c}^{\prime} \Vdash \dot{\eta}_{c} \uparrow\left(\dot{b} \backslash n_{0}\right) \equiv e .
$$

Lemma 4.2(d) implies that $\left\langle p_{c}^{\prime}: c \in U\right\rangle$ must be an antichain, which contradicts that $\mathbb{P}_{\pi}$ is ccc.

Remark 4.20: The same argument shows that, for any $g \in \prod_{\delta<\mu} B_{\delta}$ (in the ground model), $\left\{\dot{\eta}_{g(\delta)}: \delta<\mu\right\}$ witnesses $\mathrm{LCU}_{\mathbf{R}_{\mathrm{sp}}}\left(\mathbb{P}_{\pi}, \mu\right)$. 


\section{Suslin- $\lambda$-small iterations}

We now investigate suitable iterations where the iterand $\dot{\mathbb{Q}}_{\zeta}$ at step $\zeta>0$ (i.e., after the initial FS product) is

(1) either a restricted (also called partial) Suslin ccc poset (e.g., random forcing evaluated in some $V^{\mathbb{P}_{\zeta}^{-}}$for some complete subforcing $\mathbb{P}_{\zeta}^{-}$of $\mathbb{P}_{\zeta}$ );

(2) or the FS product of (in our application: at most $|\pi|$-many) $<\lambda$-size posets of reals.

More formally:

Definition 5.1: Let $\lambda$ be an uncountable cardinal. A Suslin- $\lambda$-small iteration (abbreviated $\mathrm{S} \lambda \mathrm{s}$ ) is a suitable iteration $\mathbb{P}$ with the following properties:

(S1) $\pi \backslash \pi_{1}$ is partitioned into two sets $\Sigma$ and $\Pi$.

(S2) For $\xi \in \Sigma$,

(i) $\mathbb{P}_{\xi}^{-}$is a complete subposet of $\mathbb{P}_{\xi}$,

(ii) $\mathbb{S}_{\xi}$ is a definition of a Suslin ccc poset (with parameters in the ground model),

(iii) $\dot{\mathbb{Q}}_{\xi}$ is a $\mathbb{P}_{\xi}$-name for $\left(\mathbb{S}_{\xi}\right)^{V^{\mathbb{P}_{\xi}^{-}}}$.

(S3) For $\xi \in \Pi$,

(i) $\Xi_{\xi}$ is a set in the ground model,

(ii) each element of $\Xi_{\xi}$ is a $\mathbb{P}_{\xi}$-name $\dot{Q}$ for a poset of size ${ }^{12}<\lambda$ consisting of reals,

(iii) $\dot{\mathbb{Q}}_{\xi}$ is (the $\mathbb{P}_{\xi}$-name for) the FS product of $\Xi_{\xi}$.

Remark 5.2: Regarding (S3), recall that our setting requires $\dot{\mathbb{Q}}_{\xi}$ (to be forced by $\mathbb{P}_{\xi}$ ) to be ccc (as suitable iterations have to be ccc). In contrast, in (S2), $\dot{\mathbb{Q}}_{\xi}$ will be always ccc "for free" (in $V^{\mathbb{P}_{\xi}}$ as well as in $V^{\mathbb{P}_{\xi}^{-}}$), as it is an evaluation of a Suslin ccc definition (see [JS88]).

We now show that we can replace such an iteration $\left\langle\mathbb{P}_{\zeta}^{\prime}, \dot{\mathbb{Q}}_{\zeta}^{\prime}: \zeta \in \pi\right\rangle$ with an isomorphic version $\left\langle\mathbb{P}_{\zeta}, \dot{\mathbb{Q}}_{\zeta}: \zeta \in \pi\right\rangle$ : The only difference will be in steps $\zeta \in \Pi$, where we select (hereditarily) nice names for the factors $\dot{Q} \in \Xi_{\zeta}$ and make sure that $\dot{\mathbb{Q}}_{\zeta}$ is self-indexed. In addition, we will define a dense subset $\mathbb{P}^{*}$ of hereditarily $\lambda$-small conditions, an extended "refined history domain" $\pi^{+}$, and

12 That is, each element of $\Xi_{\xi}$ is forced to have size $<\lambda$, whereas the cardinality of $\Xi_{\xi}$ may be as large as we want. 
a "refined history" $H^{*}: \mathbb{P}^{*} \rightarrow \mathcal{P}\left(\pi^{+}\right)$. These are formalized in the following notions.

Definition 5.3: Let $\lambda$ be an uncountable cardinal. A tidy Suslin- $\lambda$-small iteration is a Suslin- $\lambda$-small iteration $\mathbb{P}$ with the following additional components and properties:

(1) For $\xi \in \pi \backslash \pi_{1}, \mathbb{P}_{\xi}^{*}$ is a dense subset of $\mathbb{P}_{\xi}$.

(2) $\mathbb{P}_{\pi_{1}}^{*}=\mathbb{P}_{\pi_{1}}$.

(3) If $\xi \in \Sigma$ and $p \in \mathbb{P}_{\xi+1}^{*}$, then $p\left\lceil\xi \in \mathbb{P}_{\xi}^{*}, p(\xi)\right.$ is a nice $\mathbb{P}_{\xi}^{*}$-name of a real and $\Vdash_{\mathbb{P}_{\xi}} p(\xi) \in \mathbb{S}_{\xi} \cap V^{\mathbb{P}_{\xi}^{-}}$.

(4) For $\xi \in \Pi, \Xi_{\xi}$ is composed of nice $\mathbb{P}_{\xi}^{*}$-names for real-number-posets of size $<\lambda$ (see Definition $4.8(2)$ ). In addition, if $p \in \mathbb{P}_{\xi+1}^{*}$ then the following is satisfied:

(i) $p\left\lceil\xi \in \mathbb{P}_{\xi}^{*}\right.$.

(ii) $\operatorname{dom} p(\xi)$ is decided by $p\left\lceil\xi\right.$, that is, $p\left\lceil\xi \Vdash_{\mathbb{P}_{\xi}^{*}}\right.$ " $\operatorname{dom} p(\xi)=d_{\xi}^{p}$ " for some finite $d_{\xi}^{p} \subseteq \Xi_{\xi}$.

(iii) For each $\dot{Q} \in \operatorname{dom} p(\xi), p(\xi, \dot{Q})$ is a nice $\mathbb{P}_{\xi}^{*}$-name of a real and $\Vdash_{\mathbb{P}_{\xi}^{*}}$ $p(\xi, \dot{Q}) \in \dot{Q}$.

(iv) $p(\xi)=\langle p(\xi, \dot{Q})): \dot{Q} \in \operatorname{dom} p(\xi)\rangle$ (in particular, $p(\xi)$ is a $\mathbb{P}_{\xi}^{*}$-name).

(5) If $\pi_{1} \leq \xi<\pi$ then $\mathbb{P}_{\xi}^{*} \subseteq \mathbb{P}_{\xi+1}^{*}$.

(6) If $\gamma \in\left(\pi_{1}, \pi\right]$ is limit then

$$
\mathbb{P}_{\gamma}^{*}=\bigcup_{\xi<\gamma} \mathbb{P}_{\xi}^{*}
$$

Denote $\mathbb{P}^{*}:=\mathbb{P}_{\pi}^{*}$

Note that tidy $\mathrm{S} \lambda \mathrm{s}$ iterations are coherent in the sense that $\mathbb{P}_{\eta}^{*} \cap \mathbb{P}_{\xi}=\mathbb{P}_{\xi}^{*}$ for any $\pi_{1} \leq \xi \leq \eta \leq \pi$. Conditions (5) and (6) were included to guarantee this.

Definition 5.4: Let $\mathbb{P}$ be a tidy $\mathrm{S} \lambda \mathrm{s}$ iteration.

(1) For $\pi_{1} \leq \xi \leq \pi$ define the refined history domain

$$
\xi^{+}=\xi \cup \bigcup_{\zeta \in \xi \cap \Pi}\{\zeta\} \times \Xi_{\zeta} .
$$

(2) For $p \in \mathbb{P}^{*}$ and a $\mathbb{P}^{*}$-name $\tau$ we define the refined history $H^{*}(p) \subseteq \pi^{+}$ and $H^{*}(\tau) \subseteq \pi^{+}$as follows. For $\pi_{1} \leq \xi \leq \pi$ we define $H^{*}$ by recursion on $\xi$ for $p \in \mathbb{P}_{\xi}^{*}$ and for a $\mathbb{P}_{\xi}^{*}$-name $\tau$.

(i) For $p \in \mathbb{P}_{\pi_{1}}^{*}, H^{*}(p):=H(p)$. 
(ii) For $\xi=\zeta+1$ and $p \in \mathbb{P}_{\zeta+1}^{*}, H^{*}(p)=H^{*}(p\lceil\zeta)$ when $\zeta \notin \operatorname{supp} p$, otherwise:

- if $\zeta \in \Sigma$ then

$$
H^{*}(p):=H^{*}\left(p\lceil\zeta) \cup\{\zeta\} \cup H^{*}(p(\zeta)) ;\right.
$$

- if $\zeta \in \Pi$ then

$$
H^{*}(p):=H^{*}\left(p \lceil \zeta ) \cup \{ \zeta \} \cup ( \{ \zeta \} \times \operatorname { d o m } ( p ( \zeta ) ) ) \cup \bigcup _ { \dot { Q } \in \operatorname { d o m } ( p ( \zeta ) } \left(H^{*}(\dot{Q}) \cup H^{*}(p(\zeta, \dot{Q})) .\right.\right.
$$

(iii) When $\xi>\pi_{1}$ is limit and $p \in \mathbb{P}_{\xi}^{*}$, then $H^{*}(p)$ has already been defined (because $p \in \mathbb{P}_{\zeta}$ for some $\zeta<\xi$ ).

(iv) For any $\mathbb{P}_{\xi}^{*}$-name $\tau$ define, by $\in$-recursion,

$$
H^{*}(\tau):=\bigcup\left\{H^{*}(\sigma) \cup H^{*}(p):(\sigma, p) \in \tau\right\} .
$$

Tidy $\mathrm{S} \lambda \mathrm{s}$ iterations have many features that ease its manipulation, in particular, they have $\lambda$-small history.

Lemma 5.5: Let $\mathbb{P}$ be a tidy $S \lambda_{s}$ iteration with $\lambda$ regular. Then, for any $p \in \mathbb{P}^{*}$ :

(a) $\left|H^{*}(p)\right|<\lambda$.

(b) $H(p)=H^{*}(p) \cap \pi$.

(c) $H(\tau)=H^{*}(\tau) \cap \pi$ for any $\mathbb{P}_{\pi}^{*}$-name $\tau$.

In particular, $\mathbb{P}$ has $\lambda$-small history.

Proof. We prove (a), (b) and (c) simultaneously for all $p \in \mathbb{P}_{\xi}^{*}$ by recursion on $\pi_{1} \leq \xi \leq \pi$. It is clear that (c) follows from (b).

In the case $\xi=\pi_{1}, H^{*}(p)=\operatorname{supp} p=H(p)$, which is finite.

For the successor step $\xi=\zeta+1$, assume $\zeta \in \operatorname{supp} p$. If $\zeta \in \Sigma$ then $p(\zeta)$ is a nice $\mathbb{P}_{\zeta}^{*}$-name of a real, so it is determined by some countable $A \subseteq \mathbb{P}_{\zeta}^{*}$. Hence

$$
H^{*}(p)=H^{*}\left(p\lceil\zeta) \cup\{\zeta\} \cup \bigcup\left\{H^{*}(r): r \in A\right\}\right.
$$

so, by induction hypothesis, $\left|H^{*}(p)\right|<\lambda$.

Now assume $\zeta \in \Pi$. Since any $\dot{Q} \in \Xi_{\zeta}$ is a nice $\mathbb{P}_{\zeta}^{*}$-name for a real-numberposet of size $<\lambda$, it is determined by some $B_{\dot{Q}}$ of size $<\lambda$. Hence

$$
H^{*}(\dot{Q})=\bigcup_{s \in B_{\dot{Q}}} H^{*}(s), \text { and }\left|H^{*}(\dot{Q})\right|<\lambda,
$$


the latter by induction hypothesis. On the other hand, for any $\dot{Q} \in \operatorname{dom} p(\zeta)$, $p(\zeta, \dot{Q})$ is a $\mathbb{P}_{\zeta}^{*}$-name of a real, so it is determined by some countable $A_{\dot{Q}} \subseteq \mathbb{P}_{\zeta}^{*}$. Hence

$$
H^{*}(p(\zeta, \dot{Q}))=\bigcup_{r \in A_{\dot{Q}}} H^{*}(r),
$$

which have size $<\lambda$ by induction hypothesis. As

$$
H^{*}(p)=H^{*}\left(p\lceil\zeta) \cup\{\zeta\} \cup(\{\zeta\} \times \operatorname{dom} p(\zeta)) \cup \bigcup_{\dot{Q} \in \operatorname{dom} p(\zeta)}\left(H^{*}(\dot{Q}) \cup H^{*}(p(\zeta, \dot{Q}))\right),\right.
$$

we get $\left|H^{*}(p)\right|<\lambda$. On the other hand, since $p(\zeta)=\langle p(\zeta, \dot{Q}): \dot{Q} \in \operatorname{dom} p(\zeta)\rangle$,

$$
H(p(\zeta))=\bigcup_{\dot{Q} \in \operatorname{dom} p(\zeta)}(H(\dot{Q}) \cup H(p(\zeta, \dot{Q}))),
$$

so we can deduce (b). The limit step is immediate.

As promised, we show that any Suslin- $\lambda$-small iteration is isomorphic to a tidy one.

Lemma 5.6: If $\lambda$ is regular uncountable, then any Suslin- $\lambda$-small iteration is isomorphic to a tidy $S \lambda$ s iteration.

Proof. By recursion on $\pi_{1} \leq \xi \leq \pi$ we construct the tidy iteration up to $\mathbb{P}_{\xi}$, along with its components, and the isomorphism $i_{\xi}: \mathbb{P}_{\xi}^{\prime} \rightarrow \mathbb{P}_{\xi}$. We also guarantee that $i_{\xi}$ extends $i_{\zeta}$ for any $\pi_{1} \leq \zeta<\xi$.

CASE $\xi=\pi_{1}$ : Set $\mathbb{P}_{\pi_{1}}^{*}=\mathbb{P}_{\pi_{1}}=\mathbb{P}_{\pi_{1}}^{\prime}$ and let $i_{\pi_{1}}$ be the identity function.

CASE $\xi=\zeta+1$ With $\zeta \in \Sigma:$ As $i_{\zeta}: \mathbb{P}_{\zeta}^{\prime} \rightarrow \mathbb{P}_{\zeta}$ is an isomorphism, we let $\mathbb{P}_{\zeta}^{-}=i_{\zeta}\left[\mathbb{P}_{\zeta}^{\prime-}\right]$, which is clearly a complete subforcing of $\mathbb{P}_{\zeta}$, and evaluate $\mathbb{S}_{\zeta}$ accordingly. Note that $i_{\zeta}$ can be extended to an isomorphism $i_{\zeta+1}: \mathbb{P}_{\zeta+1}^{\prime} \rightarrow \mathbb{P}_{\zeta+1}$ in a natural way.

We define $\mathbb{P}_{\zeta+1}^{*}$ as the set of pairs $(p, \dot{q})$ where $p \in \mathbb{P}_{\zeta}^{*}, \dot{q}$ is a $\mathbb{P}_{\zeta}^{*}$ nice name for a real, and $p \Vdash \dot{q} \in \mathbb{S}_{\zeta} \cap V^{\mathbb{P}_{\zeta}^{-}}$. This is dense according to Fact 4.10(a).

CASE $\xi=\zeta+1$ With $\zeta \in \Pi$ : Fix a $\dot{Q}^{\prime}$ in $\Xi_{\zeta}^{\prime}$. As $i_{\zeta}^{*}\left(\dot{Q}^{\prime}\right)$ is forced by $\mathbb{P}_{\zeta}$ to have size $<\lambda$, according to Fact $4.10(\mathrm{~b})$, there is an equivalent $\mathbb{P}_{\zeta}^{*}$-nice name $\dot{Q}$ determined by $B_{\dot{Q}} \subseteq \mathbb{P}_{\zeta}^{*}$ of size $<\lambda$. Let $\Xi_{\zeta}$ be the set of all these names, and define $\dot{\mathbb{Q}}_{\zeta}$ to be the self-indexed FS product of the $\dot{Q}$ in $\Xi_{\zeta}$. We can obtain the isomorphism $i_{\xi+1}$ in a natural way. 
We define $\mathbb{P}_{\zeta+1}^{*}$ to consist of the $(p, \dot{q})$ as in Fact 4.14 (using $D=\mathbb{P}_{\zeta}^{*}$ ).

CASE $\xi>\pi_{1}$ LIMIT: As $\mathbb{P}$ is a FS iteration, we (have to) set $\mathbb{P}_{\xi}=\bigcup_{\zeta<\xi} \mathbb{P}_{\zeta}$; and we set $\mathbb{P}_{\xi}^{*}:=\bigcup_{\zeta<\xi} \mathbb{P}_{\zeta}^{*}$ and $i_{\xi}:=\bigcup_{\zeta<\xi} i_{\zeta}$.

For later reference, note: Assume that we can extend some $2 \mathrm{G}$-automorphism $f$ to $\mathbb{P}_{\zeta}$, and that $\dot{Q} \in \Xi_{\zeta}$. Set $\operatorname{supp}(f):=\bigcup\left\{B_{\delta}: f\left\lceil B_{\delta} \neq \operatorname{id}_{B_{\delta}}\right\}\right.$. Then

$$
\operatorname{supp}(f) \cap H^{*}(\dot{Q})=\emptyset \quad \text { implies } \hat{f}_{\xi}^{*}(\dot{Q})=\dot{Q} .
$$

This follows from Lemmas 4.12(b) and 5.5(c).

In our applications, $\mathbb{P}_{\xi}^{-}$has the following form.

Definition 5.8: Let $\mathbb{P}$ be a tidy $\mathrm{S} \lambda \mathrm{s}$ iteration. For any $X \subseteq \pi^{+}$, define

$$
\mathbb{P}^{*} \mid X:=\left\{p \in \mathbb{P}_{\pi}^{*}: H^{*}(p) \subseteq X\right\} .
$$

Note that generally $\mathbb{P}^{*} \uparrow X$ will not be a complete subforcing of $\mathbb{P}^{*}$; but we will only be interested in the case where it is; see Lemma 5.15.

Lemma 5.9: Let $\mathbb{P}$ be a tidy $S \lambda$ s iteration with $\lambda$ uncountable regular, and let $\mu \geq \lambda$ be regular and $\aleph_{1}$-inaccessible. ${ }^{13}$ If $X \subseteq \pi^{+}$and $|X|<\mu$ then $\left|\mathbb{P}^{*}\right| X \mid<\mu$.

Proof. By induction on $\xi \in\left[\pi_{1}, \pi\right]$ we show that, whenever $X \subseteq \xi^{+}$has size $<\mu$, $\left|\mathbb{P}^{*}\right| X \mid<\mu$. For $\xi=\pi_{1}$, it is clear that $\left|\mathbb{P}^{*}\right| X \mid=\max \{|\omega \times X|, 1\}<\mu$.

For limit $\xi>\pi_{1}, \mathbb{P}^{*} \uparrow X=\bigcup_{\eta \in c} \mathbb{P}^{*} \uparrow\left(X \cap \eta^{+}\right)$where $c$ is a cofinal subset of $\xi$ of size $\operatorname{cof}(\xi)$. If $\operatorname{cof}(\xi)<\mu$ then $\left|\mathbb{P}^{*}\right| X \mid<\mu$ because it is a union of $<\mu$ many sets of size $<\mu$; if $\operatorname{cof}(\xi) \geq \mu$ then $X \subseteq \eta^{+}$for some $\eta<\xi$, so $\left|\mathbb{P}^{*}\right| X \mid<\mu$ by induction hypothesis.

For the successor step $\xi=\zeta+1$, assume $X \subseteq(\zeta+1)^{+}$and $X \nsubseteq \zeta^{+}$(the non-trivial case). Put $X_{0}:=X \cap \zeta^{+}$. By induction hypothesis, $\left|\mathbb{P}^{*}\right| X_{0} \mid<\mu$ and, since $\mu$ is $\aleph_{1}$-inaccessible, there are at most $\left.\left|\mathbb{P}^{*}\right| X_{0}\right|^{\aleph_{0}}<\mu$ many nice $\mathbb{P}^{*} \mid X_{0}$-names of reals.

Let $p \in \mathbb{P}^{*} \uparrow X$. If $\xi \in \Sigma$ then $p(\xi)$ is a nice $\mathbb{P}^{*} \uparrow X_{0}$-name of a real; if $\xi \in \Pi$, then $p(\xi)$ is determined by a finite partial function from $\left(\{\xi\} \times \Xi_{\xi}\right) \cap X$ into the set of nice $\mathbb{P}^{*} \mid X_{0}$-names of reals, and there are $<\mu$-many such finite partial functions. Hence, $\left|\mathbb{P}^{*}\right| X \mid<\mu$.

13 Recall that a cardinal $\mu$ is $\kappa$-inaccessible if $\theta^{\nu}<\mu$ for any cardinals $\theta<\mu$ and $\nu<\kappa$. 
Corollary 5.10: Let $\mathbb{P}$ be a tidy $S \lambda$ s iteration with $\lambda$ regular.

(a) $\mathbb{P}$ has $\lambda$-small history.

(b) Every $p \in \mathbb{P}^{*}$ is an element of $\mathbb{P}^{*} \mid X$ from some $X \subseteq \pi^{+}$of size $<\lambda$.

(c) For every $\mathbb{P}$-name of a real there is an equivalent $\mathbb{P}^{*} \uparrow X$-name for some $X \subseteq \pi^{+}$of size $<\lambda$.

(d) Assume that $|\pi|^{\aleph_{0}}=|\pi|$, that $\lambda \leq|\pi|^{+}$, and that $\left|\Xi_{\zeta}\right| \leq|\pi|$ for each $\zeta \in \Pi$. Then $\left|\mathbb{P}^{*}\right| \leq|\pi|$.

Proof. (a) follows from Lemma 5.5(a),(b); (b) follows from Lemma 5.5(a) (using $X:=H^{*}(p)$ ); and (c) follows from (b) (use a nice $\mathbb{P}^{*}$-name for a real).

For (d), set $\mu=|\pi|^{+}$(the cardinal successor). Note that $\mu$ is $<\aleph_{1}$-inaccessible because $|\pi|^{\aleph_{0}}=|\pi|$, and

$$
\left|\pi^{+}\right| \leq|\pi| \times \sup _{\zeta \in \Pi}\left\{\left|\Xi_{\zeta}\right|\right\} \leq|\pi|<\mu .
$$

Therefore $\left|\mathbb{P}^{*}\right|=\left|\mathbb{P}^{*}\right| \pi^{+} \mid<\mu$ by Lemma 5.9 (for $X=\pi^{+}$).

In our applications, $\mathbb{P}_{\xi}^{-}=\mathbb{P}^{*} \uparrow C_{\xi}$ with $C_{\xi} \subseteq \xi^{+}$for all $\xi \in \Sigma$. We will now show how to build symmetric $\mathrm{S} \lambda \lambda_{\text {s-iterations: }}$

Definition 5.11: Let $\mathbb{P}$ be a tidy S $\lambda$ s iteration, and let $h: \pi_{1} \rightarrow \pi_{1}$ be a $2 \mathrm{G}-$ automorphism.

(1) Let $\xi \in \Pi$. We say that $\Xi_{\xi}$ is closed if, whenever $h \in \mathcal{H}^{*}$ and $\hat{h}_{\xi}$ can be defined (see Definition 4.11), $\dot{Q} \in \Xi_{\xi}$ implies $\hat{h}_{\xi}^{*}(\dot{Q}) \in \Xi_{\xi}$ (where $\mathcal{H}^{*}$ is the group of 2G-automorphisms fixed in Definition 4.11(5)).

(2) We say that $C \subseteq \pi^{+}$is closed if, for any $h \in \mathcal{H}^{*}$, it satisfies:

(i) For any $\delta<\pi_{0}, B_{\delta} \cap C \neq \emptyset$ implies $B_{\delta} \subseteq C$.

(ii) For any $\xi \in \Pi$, whenever $\hat{h}_{\xi}$ can be defined, if $(\xi, \dot{Q}) \in C$ then $\left(\xi, \hat{h}_{\xi}^{*}(\dot{Q})\right) \in C$ and $\xi \in C$.

Lemma 5.12: Assume that $\mathbb{P}$ is a tidy $S \lambda_{s}$ iteration such that the following requirements are satisfied:

(I) For any $\xi \in \Pi, \Xi_{\xi}$ is closed.

(II) For any $\xi \in \Sigma, \mathbb{P}_{\xi}^{-}=\mathbb{P}^{*} \mid C_{\xi}$ where $C_{\xi} \subseteq \xi^{+}$is closed.

Then we get:

(a) $\mathbb{P}$ is symmetric (i.e., $h$-symmetric for all $h \in \mathcal{H}^{*}$ ).

(b) $\hat{h}\left[\mathbb{P}^{*}\lceil C]=\mathbb{P}^{*}\left\lceil C\right.\right.$ for all closed $C \subseteq \pi^{+}$. 
Of course we will have to also make sure that the assumption " $\mathbb{P}$ is a tidy $\mathrm{S} \lambda \mathrm{s}$-iteration" is satisfied. The nontrivial points of these assumptions are:

(I-b) For $\zeta \in \Pi$ the FS product $\mathbb{Q}_{\zeta}$ is ccc. (In our case this will be trivial, as all factors $\dot{Q}$ will be Knaster.)

(II-b) For $\zeta \in \Sigma, \mathbb{P}^{*}\left\lceil C_{\zeta}\right.$ is a complete subforcing of $\mathbb{P}^{*}$. We will see in Lemma 5.15 how to achieve this.

Proof. By induction on $\xi \in\left[\pi_{1}, \pi\right]$ we show that $\hat{h}_{\xi}$ can be defined for any $h \in \mathcal{H}^{*}$ (towards (a)), and that (b) is valid for any closed $C \subseteq \xi^{+} .{ }^{14}$

CASE $\xi=\pi_{1}$ : It is clear that $\hat{h}_{\pi_{1}}$ can be defined; (b) is clear because $h\left[B_{\delta}\right]=B_{\delta}$ for any $\delta<\pi_{0}$ and $h \in \mathcal{H}^{*}$.

CASE $\xi=\zeta+1$ WITH $\zeta \in \Sigma:(a)$ : Note that

$$
\Vdash \hat{h}_{\zeta}^{*}\left(\mathbb{Q}_{\zeta}\right)=\hat{h}_{\zeta}^{*}\left(\mathbb{S}_{\zeta}^{V^{\mathbb{P}^{*}\left\lceil C_{\zeta}\right.}}\right)=\mathbb{S}_{\zeta}^{V^{\hat{h}_{\zeta}\left[\mathbb{P}^{*} \mid C_{\zeta}\right]}}
$$

(as $\mathbb{S}_{\zeta}$ only uses parameters from the ground model), which is $\dot{\mathbb{Q}}_{\zeta}$ by induction hypothesis (as we assume that $C_{\zeta}$ is closed). So we can extend $\hat{h}_{\zeta}$ to $\hat{h}_{\zeta+1}$.

(b): Note that $\xi^{+}=\zeta^{+} \cup\{\zeta\}$, so if $C \subseteq \xi^{+}$is closed (and not already a subset of $\left.\zeta^{+}\right)$, then $C=C^{\prime} \cup\{\zeta\}$ with $C^{\prime}$ closed, and $(p, \dot{q}) \in \mathbb{P}^{*} \uparrow C$ means that $p \in \mathbb{P}^{*} \uparrow C^{\prime}$ and $\dot{q}$ is a nice $\mathbb{P}^{*}\left\lceil C^{\prime}\right.$-name for an element of $\dot{\mathbb{Q}}_{\zeta}$. Then $\hat{h}_{\zeta}^{*}(\dot{q})$ is a nice $\hat{h}_{\zeta}\left[\mathbb{P}^{*}\left\lceil C^{\prime}\right]\right.$-name for an element of $\hat{h}_{\zeta}^{*}\left(\dot{\mathbb{Q}}_{\zeta}\right)$, which is by induction hypothesis a nice $\mathbb{P}^{*}\left\lceil C^{\prime}\right.$-name for an element of $\dot{\mathbb{Q}}_{\zeta}$, i.e., $\hat{h}_{\zeta+1}((p, \dot{q})) \in \mathbb{P}^{*}\lceil C$. This shows that $\hat{h}_{\xi}\left[\mathbb{P}^{*} \uparrow C\right] \subseteq \mathbb{P}^{*} \uparrow C$. As this is also true for the inverse of $h$ (because $\left.h^{-1} \in \mathcal{H}^{*}\right)$, by Lemma $4.12(\mathrm{c})$ we get equality.

CASE $\xi=\zeta+1$ WITh $\zeta \in \Pi$ : First note that, by induction hypothesis, $\hat{h}_{\zeta}\left[\mathbb{P}_{\zeta}^{*}\right]=\mathbb{P}_{\zeta}^{*}$ because $\mathbb{P}_{\zeta}^{*}=\mathbb{P}^{*}\left\lceil\zeta^{+}\right.$and $\zeta^{+}$is closed.

(a): Since $\dot{\mathbb{Q}}_{\zeta}$ is a $\mathbb{P}_{\zeta}^{*}$-name for the self-indexed FS product of the (evaluated) set $\Xi_{\zeta}=\left\{\dot{Q}: \dot{Q} \in \Xi_{\zeta}\right\}, \hat{h}_{\zeta}^{*}\left(\dot{\mathbb{Q}}_{\zeta}\right)$ is the $\mathbb{P}_{\zeta}^{*}$-name for the self-indexed FS product of the (evaluated) set

$$
\hat{h}_{\zeta}^{*}\left[\Xi_{\zeta}\right]=\left\{\hat{h}_{\zeta}^{*}(\dot{Q}): \dot{Q} \in \Xi_{\zeta}\right\}
$$

But as the ground model set of names $\left\{\hat{h}^{*}(\dot{Q}): \dot{Q} \in \Xi_{\zeta}\right\}$ is identical to the ground model set of names $\Xi_{\zeta}$, their evaluations are identical as well (because $\Xi_{\xi}$ is closed under inverses, and by Lemma $4.12(\mathrm{c}))$. In other words, $\hat{h}_{\zeta}^{*}\left(\dot{\mathbb{Q}}_{\zeta}\right)=\dot{\mathbb{Q}}_{\zeta}$, and $\hat{h}_{\zeta}$ can be extended to $\mathbb{P}_{\xi}$.

14 In this proof we only use that $h \in \mathcal{H}^{*}$ implies $h^{-1} \in \mathcal{H}^{*}$. 
(b): Assume that $C \subseteq \xi^{+}=\zeta^{+} \cup\{\zeta\} \cup\left(\{\zeta\} \times \Xi_{\zeta}\right)$ is closed, and that $(p, \dot{q}) \in \mathbb{P}^{*} \mid C$ (but to avoid the trivial case, not in $\mathbb{P}_{\zeta}^{*}$ ). This means that $p \in \mathbb{P}^{*}\left\lceil C^{\prime}\right.$ for $C^{\prime}=C \cap \zeta^{+}$, and it determines $\operatorname{dom}(\dot{q})=\left\{\dot{Q}_{1}, \ldots, \dot{Q}_{n}\right\}$, such that all $\left(\zeta, \dot{Q}_{i}\right)$ are in $C$ (and each $\dot{q}\left(\dot{Q}_{i}\right)$ is a nice $\mathbb{P}^{*}\left\lceil C^{\prime}\right.$-name). Then $\hat{h}_{\zeta}(p) \in \mathbb{P}^{*} \uparrow C^{\prime}$ by induction hypothesis, and it determines $\operatorname{dom}\left(\hat{h}_{\zeta}^{*}(\dot{q})\right)=\left\{\hat{h}_{\zeta}^{*}\left(\dot{Q}_{1}\right), \ldots, \hat{h}_{\zeta}^{*}\left(\dot{Q}_{n}\right)\right\}$ (and each $\hat{h}_{\zeta}^{*}(\dot{q})\left(\dot{Q}_{i}\right)$ is a $\mathbb{P}^{*}\left\lceil C^{\prime}\right.$-nice name). Accordingly $\hat{h}_{\xi}((p, \dot{q})) \in \mathbb{P}^{*}\lceil C$ as required.

We conclude that $\hat{h}_{\xi}\left[\mathbb{P}^{*} \mid C\right] \subseteq \mathbb{P}^{*} \uparrow C$, but equality holds because the same is true for $h^{-1}$.

CASE $\xi$ LIMIT: By induction hypothesis, $\hat{h}_{\zeta}$ is defined for all $\zeta<\xi$, so $\hat{h}_{\xi}$ is defined (as its union). On the other hand, if $C \subseteq \xi^{+}$is closed then

$$
C=\bigcup_{\zeta<\xi} C \cap \zeta^{+}
$$

where each $C \cap \zeta^{+}$is closed, so $\hat{h}_{\xi}\left[\mathbb{P}^{*}\lceil C]=\mathbb{P}^{*}\lceil C\right.$ by induction hypothesis.

We address some few facts about closed sets.

Lemma 5.13: Let $\mathbb{P}$ be a symmetric tidy $S \lambda$ s iteration. Then:

(a) The union of closed sets is closed.

(b) If $A \subseteq \pi^{+}$has size $<\mu$, with $\mu \geq \max \left\{\lambda, \aleph_{2}\right\}$ uncountable regular, then the closure $\bar{A}$ of $A$ (the smallest closed set containing $A$ ) has size $<\mu$.

Proof. Property (a) is straightforward. We show by induction on $\xi \in\left[\pi_{1}, \pi\right]$ that (b) holds for any $A \subseteq \xi^{+}$.

CASE $\xi=\pi_{1}: \bar{A}=\bigcup\left\{B_{\delta}: B_{\delta} \cap A \neq \emptyset\right\}$. So $|\bar{A}|=\aleph_{1} \times|A|<\mu$.

CASE $\xi=\zeta+1$ with $\zeta \in \Sigma$ : If $A \subseteq \xi^{+}$has size $<\mu$, then $\bar{A} \subseteq \overline{\left(A \cap \zeta^{+}\right)} \cup\{\zeta\}$ has size $<\mu$.

CASE $\xi=\zeta+1$ WITH $\zeta \in \Pi$ : For $h \in \mathcal{H}^{*}$, set

$$
\operatorname{supp}(h)=\bigcup\left\{B_{\delta}: h\left\lceil B_{\delta} \neq \mathrm{id}_{B_{\delta}}\right\} .\right.
$$

Let $A^{*}$ be the closure of

$$
\bigcup\left\{H^{*}(\dot{Q}) \cap \pi_{1}:(\zeta, \dot{Q}) \in A\right\} .
$$

$H^{*}(\dot{Q})$ has size $<\lambda \leq \mu$ by Lemma $5.5(\mathrm{c})$, and therefore also the set $A^{*}$ has size $<\mu$. 
It is clear that $\bar{A} \subseteq \overline{\left(A \cap \zeta^{+}\right)} \cup\{\zeta\} \cup X$ for

$$
X:=\left\{\left(\zeta, \hat{h}^{*}(\dot{Q})\right):(\zeta, \dot{Q}) \in A, h \in \mathcal{H}^{*}\right\} .
$$

Since $\mathcal{H}^{*}$ is a group, $\{\zeta\} \cup X$ is closed. We claim that we get the same set $X$ if we replace $\mathcal{H}^{*}$ with

$$
\mathcal{H}^{\prime}:=\left\{g \in \mathcal{H}^{*}: \operatorname{supp}(g) \subseteq A^{*}\right\} .
$$

As $\mathcal{H}^{\prime}$ has size $<\mu\left(\right.$ recall that $|\operatorname{supp}(g)| \leq \aleph_{1}$ for any $g \in \mathcal{H}^{*}$ ), we get $|\bar{A}|<\mu$, as required.

Note that for $f \in \mathcal{H}^{*}$ and $(\zeta, \dot{Q}) \in A$, by (5.7) $\operatorname{supp}(f) \cap A^{*}=\emptyset$ implies

$$
\hat{f}_{\zeta}^{*}(\dot{Q})=\dot{Q}
$$

And for $h \in \mathcal{H}^{*}$, there is a $g \in \mathcal{H}^{\prime}$ such that $f:=g^{-1} \circ h$ satisfies

$$
\operatorname{supp}(f) \cap A^{*}=\emptyset .
$$

(Basically, $g\left\lceil A^{*}=h \uparrow A^{*}\right.$ and $g \uparrow\left(\pi_{1} \backslash A^{*}\right)$ is the identity.) So $\hat{f}^{*}(\dot{Q})=\dot{Q}$, which implies

$$
\hat{g}^{*}(\dot{Q})=\hat{g}^{*}\left(\hat{f}^{*}(\dot{Q})\right)=\hat{h}^{*}(\dot{Q})
$$

as required.

Case $\xi$ Limit: If $\operatorname{cof}(\xi) \geq \mu$, then $A \subseteq \zeta^{+}$for some $\zeta<\xi$, so $|\bar{A}|<\mu$ by induction hypothesis. Otherwise, $\bar{A}=\bigcup_{\zeta \in I} \overline{A \cap \zeta^{+}}$for some witness $I$ of $\operatorname{cof}(\xi)$, so again $|\bar{A}|<\mu$.

Lemma 5.14: To satisfy assumption (I) of Lemma 5.12 for $\xi \in \Pi$, the following is sufficient, while assuming (I) and (II) for $\zeta<\xi$ :

(I') For some formula $\varphi(x, y)$ using only parameters from the ground model and some $\kappa_{\xi} \leq \lambda, \Xi_{\xi}$ is the set of all nice $\mathbb{P}_{\xi}^{*}$-names $\dot{Q}$ for $<\kappa_{\xi}$-sized forcings consisting of reals such that $\Vdash_{\mathbb{P}_{\xi}^{*}} \varphi(\dot{Q}, \xi)$.

Proof. By the assumption and Lemma 5.12, $\mathbb{P}_{\xi}$ is symmetric and $h_{\xi}^{*}\left[\mathbb{P}_{\xi}^{*}\right]=\mathbb{P}_{\xi}^{*}$ for any $h \in \mathcal{H}^{*}$ (because $\mathbb{P}_{\xi}^{*}=\mathbb{P}^{*} \mid \xi^{+}$).

Let $\dot{Q}$ be such a nice $\mathbb{P}_{\xi}^{*}$-name. Then $\hat{h}_{\xi}^{*}(\dot{Q})$ is also a nice $\mathbb{P}_{\xi}^{*}$-name, and $\Vdash_{\mathbb{P}_{\xi}^{*}} \varphi\left(\hat{h}_{\xi}^{*}(\dot{Q}), \xi\right)$ as $\varphi$ only uses ground model parameters (i.e., standard names).

As mentioned, we need closed $C \subseteq \pi^{+}$that define complete subforcings. For this we use the following result: 
Lemma 5.15: Let $\mathbb{P}$ be as in Lemma 5.12, and let $\mu>\lambda$ be regular and $\aleph_{1^{-}}$ inaccessible.

(a) For $A \subseteq \pi^{+}$of size $<\mu$ there is some closed $C \supseteq A$ of size $<\mu$, such that $\mathbb{P}^{*} \mid C \lessdot \mathbb{P}^{*}$.

(b) The closed sets $C \in\left[\pi^{+}\right]^{<\mu}$ that satisfy $\mathbb{P}^{*}\left\lceil C \lessdot \mathbb{P}^{*}\right.$ form a $\lambda$-club.

The proof is just a standard Skolem-Löwenheim type closure argument:

Proof. (a) Using Corollary 5.10(b) we can fix a function $f:\left(\mathbb{P}_{\pi}^{*}\right)^{2} \rightarrow\left[\pi^{+}\right]^{<\lambda}$ such that if $p$ and $q$ are compatible then there is some $r \leq p, q$ in $\mathbb{P}^{*}\lceil f(p, q)$. Also, we can fix a function $g:\left(\mathbb{P}_{\pi}^{*}\right) \leq \omega \rightarrow\left[\pi^{+}\right]^{<\lambda}$ such that, whenever

$$
\bar{p}=\left\langle p_{n}: n<w\right\rangle \quad(w \leq \omega)
$$

is a non-empty antichain but not maximal in $\mathbb{P}_{\pi}$, then there is some $q \in \mathbb{P}^{*} \mid g(\bar{p})$ with $q \perp p_{n}$ for any $n<w$. By recursion on $\alpha<\lambda$, define

$$
A_{\alpha}^{\prime}:=A \cup A_{<\alpha} \cup \bigcup_{p, q \in \mathbb{P}^{*} \uparrow A_{<\alpha}} f(p, q) \cup \bigcup_{\bar{p} \in\left(\mathbb{P}^{*} \mid A_{<\alpha}\right) \leq \omega} g(\bar{p})
$$

where

$$
A_{<\alpha}:=\bigcup_{\xi<\alpha} A_{\xi}
$$

and let $A_{\alpha}:=\overline{A_{\alpha}^{\prime}}$ be the closure (see Lemma 5.13). So $\left|A_{\alpha}\right|<\mu$, and we can set $C:=\bigcup_{\alpha<\lambda} A_{\alpha}$, which is as desired because any countable sequence in $\mathbb{P}^{*} \mid C$ is a countable sequence in $\mathbb{P}^{*}\left\lceil A_{\alpha}\right.$ for some $\alpha<\lambda$.

(b) Let $\left\langle B_{i}: i \in \lambda\right\rangle$ be an increasing sequence of closed subsets of $\pi^{+}$ such that $\mathbb{P}^{*} \mid B_{i}$ is a complete subforcing of $\mathbb{P}^{*}$. Set $B:=\bigcup_{i \in \lambda} B_{i}$. According to $5.13(\mathrm{a}) B$ is closed. Assume that $A \subseteq \mathbb{P}^{*} \mid B$ is a maximal antichain. Any $p, q \in A$ are incompatible in $\mathbb{P}^{*} \mid B_{i}$ for some $i$, and therefore in $\mathbb{P}^{*}$. Due to ccc, $A$ is countable, and by Corollary 5.10(b) there is an $i<\lambda$ such that $A \subseteq \mathbb{P}^{*} \mid B_{i}$. Therefore $A$ is maximal in $\mathbb{P}^{*}$.

Corollary 5.16: With the same hypothesis of Lemma 5.15, if $P \subseteq \mathbb{P}_{\pi}^{*}$ has size $<\mu$, then there is some $\mathbb{P}^{-} \lessdot \mathbb{P}_{\pi}^{*}$ of size $<\mu$ such that

$$
P \subseteq \mathbb{P}^{-} .
$$

Proof. Apply Lemma 5.15 to $A:=\bigcup_{p \in P} H^{*}(p)$. 
We now summarize what we already know about the construction that we are going to perform in the next section:

COROLlaRY 5.17: Let $\lambda$ be regular uncountable and assume $\lambda \leq|\pi|,|\pi|<\lambda=|\pi|$, and that $\pi \backslash \pi_{1}$ is partitioned into $\Sigma$ and $\Pi$. We inductively construct a (tidy) $S \lambda$ s iteration $\mathbb{P}$ as follows:

$(\Sigma)$ As step $\zeta \in \Sigma$, we pick a (definition of a) Suslin-ccc forcing $\mathbb{S}_{\zeta}$, some $\aleph_{1}$-inaccessible $\kappa_{\zeta}>\lambda$, and some $C_{\zeta}$ in the $\lambda$-club set $\left[\zeta^{+}\right]^{<\kappa_{\zeta}}$ of Lemma 5.15, and set $\mathbb{Q}_{\zeta}=\mathbb{S}^{V^{\mathbb{P}^{*} \mid C_{\zeta}}} \cdot\left(\right.$ So $\mathbb{P}_{\zeta}^{-}=\mathbb{P}^{*} \uparrow C_{\zeta}$.)

(II) Fix a formula $\varphi(x, y)$ with parameters in the ground model. At step $\zeta \in \Pi$, pick some regular uncountable $\kappa_{\zeta} \leq \lambda$ and let $\dot{\mathbb{Q}}_{\zeta}$ be (a suitable name for) the FS product of all Knaster real-number-posets of size $<\kappa_{\zeta}$ satisfying $\varphi(x, \zeta)$.

Then (inductively) $\mathbb{P}_{\xi}$ is a well defined ccc forcing for $\pi_{1} \leq \xi \leq \pi$, and

(a) $\operatorname{LCU}_{\mathrm{sp}}\left(\mathbb{P}_{\xi}, \mu\right)$ holds for any regular $\lambda \leq \mu \leq \pi_{0}$,

(b) $\mathbb{P}_{\xi}$ forces that the continuum has size below $|\pi|$.

Proof. Each $\dot{\mathbb{Q}}_{\zeta}$ is forced to be ccc (by either absoluteness or the Knaster assumption), so we get a valid iteration (and we assume that we choose the names for the iterands such that we get a tidy $\mathrm{S} \lambda \mathrm{s}$-iteration).

LCU follows from Lemmas 5.14 and 5.12(a), and Theorem 4.19.

For the size of the continuum, we use Corollary 5.10(d) to show by induction that $\left|\mathbb{P}_{\xi}^{*}\right| \leq|\pi|$ : Assume this already is the case for $\zeta \in \Pi$, then $\Xi_{\zeta}$ consists of nice $\mathbb{P}_{\zeta}^{*}$-names for $<\lambda$-sized real-number-forcings, and there are only $\left|\mathbb{P}_{\zeta}^{*}\right|^{<\lambda} \leq|\pi|$ many such nice names.

\section{The forcing construction for the left hand side}

In this section, we prove the first step of the main theorem: Theorem 6.11, which gives the independence results for the left hand side. After the work we have done in the previous sections, this is basically a simple variant of the construction in [GKS19]. 


\section{A. Preliminaries.}

Notation 6.1: Denote

$$
\left(\mathfrak{b}_{i}, \mathfrak{d}_{i}\right):= \begin{cases}(\operatorname{add}(\mathcal{N}), \operatorname{cof}(\mathcal{N})) & \text { if } i=1, \\ (\operatorname{cov}(\mathcal{N}), \operatorname{non}(\mathcal{N})) & \text { if } i=2, \\ (\mathfrak{b}, \mathfrak{d}) & \text { if } i=3, \\ (\operatorname{non}(\mathcal{M}), \operatorname{cov}(\mathcal{M})) & \text { if } i=4, \\ (\mathfrak{s}, \mathfrak{r}) & \text { if } i=\mathrm{sp} .\end{cases}
$$

As in [GKS19, GKMSa], for $i \in\{1,2,3,4, \mathrm{sp}\}$ consider Blass-uniform relational systems $\mathbf{R}_{i}^{\mathrm{LCU}}$ and $\mathbf{R}_{i}^{\mathrm{COB}}$ such that, following in Example 3.1, $\mathbf{R}_{\mathrm{sp}}^{\mathrm{LCU}}=\mathbf{R}_{\mathrm{sp}}^{\mathrm{COB}}=\mathbf{R}_{\mathrm{sp}}$ and ZFC proves $^{15}$

$$
\mathfrak{b}\left(\mathbf{R}_{i}^{\mathrm{COB}}\right) \leq \mathfrak{b}_{i} \leq \mathfrak{b}\left(\mathbf{R}_{i}^{\mathrm{LCU}}\right) \text { and } \mathfrak{d}\left(\mathbf{R}_{i}^{\mathrm{LCU}}\right) \leq \mathfrak{d}_{i} \leq \mathfrak{d}\left(\mathbf{R}_{i}^{\mathrm{COB}}\right)
$$

We abbreviate $\mathrm{COB}_{\mathbf{R}_{i}^{\text {сов }}}$ by $\mathrm{COB}_{i}$, and $\mathrm{LCU}_{\mathbf{R}_{i}^{\mathrm{LCU}}}$ by $\mathrm{LCU}_{i}$.

For completeness, we review the posets we use in our construction.

Definition 6.2: Define the following forcing notions (where the forcing in item $(i)$ is designed to increase $\mathfrak{b}_{i}$ ):

(1) Amoeba forcing $\mathbb{A}$ is the poset whose conditions are subtrees $T \subseteq 2^{<\omega}$ without maximal nodes such that $[T]$, the set of branches of $T$, has measure $<\frac{1}{2}$ (with respect to the Lebesgue measure of $2^{\omega}$ ). The order is $\supseteq$.

(2) Random forcing $\mathbb{B}$ is the poset whose conditions are subtrees $T \subseteq 2^{<\omega}$ without maximal nodes such that $[T]$ has positive measure. The order is $\subseteq$.

(3) Hechler forcing is $\mathbb{D}:=\omega^{<\omega} \times \omega^{\omega}$ ordered by $(t, y) \leq(s, x)$ iff $s \subseteq t, x \leq y$ (pointwise) and $t(i) \geq x(i)$ for all $i \in|t| \backslash|s|$.

(4) Eventually different forcing is

$$
\mathbb{E}:=\omega^{<\omega} \times \bigcup_{n<\omega}\left([\omega]^{\leq n}\right)^{\omega}
$$

ordered by $(t, \psi) \leq(s, \varphi)$ iff $s \subseteq t, \forall i<\omega(\varphi(i) \subseteq \psi(i))$ and $t(i) \notin \varphi(i)$ for all $i \in|t| \backslash|s|$.

(sp) Let $F$ be a base of a (free) filter on $\omega$. Mathias-Prikry forcing on $F$ is $\mathbb{M}_{F}:=\left\{(s, x) \in[\omega]<\aleph_{0} \times F: \max (s)<\min (x)\right\}($ here $\max (\emptyset):=-1)$ ordered by $(t, y) \leq(s, x)$ if $s \subseteq t, y \subseteq x$ and $t \backslash s \subseteq x$.

15 In more detail, $\mathbf{R}_{i}^{\mathrm{LCU}}=\mathbf{R}_{i}^{\mathrm{COB}}$ except when $i=2$. If we follow [BCM21] we can also consider $\mathbf{R}_{2}^{\mathrm{LCU}}=\mathbf{R}_{2}^{\mathrm{COB}}$. 
For each of the posets above it is easy to construct a 1-1 function from the poset into $\omega^{\omega}$. So, until the end of this section, the posets above are seen as subsets of $\omega^{\omega}$. Moreover, the posets (1)-(4) are Suslin ccc, and they are homeomorphic to a Borel subset of $\omega^{\omega}$ (and the order is Borel as well).

In the proof of Theorem 6.6 we deal with special restrictions of the posets (1)-(4) under sets of reals of the following form.

Definition 6.3: Let $\lambda \geq \aleph_{1}$ be a cardinal. Say that $E \subseteq \omega^{\omega}$ is $\lambda$-elementary if $E=\omega^{\omega} \cap N$ for some regular $\chi \geq\left(2^{\aleph_{0}}\right)^{+}$and some $N \preceq \mathcal{H}_{\chi}$ of size $<\lambda$, where $\mathcal{H}_{\chi}$ denotes the collection of hereditarily $<\lambda$-size sets.

We look at posets of the form $\mathbb{S} \cap E$ where $\mathbb{S}$ is a poset as in (1)-(4) and $E \subseteq \omega^{\omega}$ is $\lambda$-elementary. Note that, whenever $\chi \geq\left(2^{\aleph_{0}}\right)^{+}, N \preceq \mathcal{H}_{\chi}$ and $E=\omega^{\omega} \cap N$, we have $\mathbb{S} \cap E=\mathbb{S}^{N}$. Therefore:

FACT 6.4: Let $E \subseteq \omega^{\omega}$ be elementary. Then:

(1) The poset $\mathbb{A} \cap E$ adds a (code of a) Borel measure zero set that contains all Borel null sets with Borel code in $E$.

(2) The generic real added by $\mathbb{B} \cap E$ evades all Borel null sets with Borel code in $E$.

(3) The generic real added by $\mathbb{D} \cap E$ dominates all the functions in $E$.

(4) The generic real added by $\mathbb{E} \cap E$ is eventually different from all the functions in $E$.

We now show how to modify the forcing construction in [GKMSb, §4 \& §5] to include $\mathrm{LCU}_{\mathrm{sp}}$ and $\mathrm{COB}_{\mathrm{sp}}$, by performing a construction according to the previous section, in particular to Corollary 5.17. We will assume the following:

Assumption 6.5: $k_{0} \in[2, \omega] ; \lambda_{\mathfrak{m}} \leq \lambda_{1} \leq \lambda_{2} \leq \lambda_{3}<\lambda_{4}$ are uncountable regular cardinals, $\lambda_{5} \geq \lambda_{4}$ is a cardinal, $\lambda_{3}=\chi^{+}, \lambda_{\mathfrak{m}} \leq \lambda_{\mathrm{sp}} \leq \lambda_{3}$ regular, such that $\chi^{<\chi}=\chi \geq \aleph_{1}, \lambda_{5}^{<\lambda_{4}}=\lambda_{5}$, and $\lambda_{i}$ is $\aleph_{1}$-inaccessible whenever $\lambda_{i}>\lambda_{\mathrm{sp}}$ and $1 \leq i \leq 4$.

Our intention is to show the following:

GoAL 6.6: There is a ccc poset $\mathbb{P}$ of size $\lambda_{5}$ such that, for any $i \in\{1,2,3,4, \mathrm{sp}\}$,

(a) $\mathrm{LCU}_{i}(\mathbb{P}, \theta)$ holds for any regular $\lambda_{i} \leq \theta \leq \lambda_{5}$,

(b) there is some directed $S_{i}$ with $\mathrm{cp}\left(S_{i}\right)=\lambda_{i}$ and $\left|S_{i}\right|=\lambda_{5}$ such that $\mathrm{COB}_{i}\left(\mathbb{P}, S_{i}\right)$ holds, 
(c) $\mathbb{P}$ forces $\mathfrak{p}=\mathfrak{s}=\lambda_{\mathrm{sp}}$ and $\mathfrak{c}=\lambda_{5}$,

(d) $\mathbb{P}$ forces $\mathfrak{m}_{k}=\aleph_{1}$ for any $k \in\left[1, k_{0}\right)$, and $\mathfrak{m}_{k}=\lambda_{\mathfrak{m}}$ for any $k \in\left[k_{0}, \omega\right] .{ }^{16}$

The way to achieve this is parallel to [GKS19, §1]: As a first step we give the "basic construction" in Lemma 6.8, using "simple bookkeeping" (which is described by parameters $\bar{C}=\left\langle C_{\alpha}\right\rangle_{\alpha \in \Sigma}$ in the ground model). This gives us everything apart from $\mathrm{LCU}_{3}$ (i.e., we do not claim that $\mathfrak{b}$ remains small). This first step contains the only new aspect of the construction: As we use a variant of the construction according to the previous section, we get $\mathrm{LCU}_{\mathrm{sp}}$.

The next steps are just as in [GKS19, §1.3 \& §1.4]. In Lemma 6.10 we remark: Assuming $2^{\chi} \geq \lambda_{5}$ (in addition to Assumption 6.5), we can choose the bookkeeping parameters $\bar{C}$ in such a way that the resulting forcing satisfies $\mathrm{LCU}_{3}$ and thus all of Goal 6.6.

And finally we show Theorem 6.11: without the assumption $2^{\chi} \geq \lambda_{5}$ (while assuming 6.5) we can also get all of Goal 6.6. Why do we need to supress the assumption $2^{\chi} \geq \lambda_{5}$ from Lemma 6.10? Because we can then additionally control the right-hand side characteristics in Section 7, using the method of elementary submodels from [GKMSa].

In the following proof, we deal with the case $2 \leq k_{0}<\omega$ and $\lambda_{\mathfrak{m}}>\aleph_{1}$. In Section 6.D we mention the necessary changes for the remaining cases.

6.B. The BAsic forcing construction. To each $1 \leq i \leq 4$ associate a Suslin ccc poset as follows: $\mathbb{S}_{1}=\mathbb{A}, \mathbb{S}_{2}=\mathbb{B}, \mathbb{S}_{3}=\mathbb{D}$, and $\mathbb{S}_{4}=\mathbb{E}$.

Set $\lambda:=\lambda_{\mathrm{sp}}$. Let $i^{*}$ be the minimal $i$ such that $\lambda_{i}>\lambda$. Note that $1 \leq i^{*} \leq 4$. Set $I_{1}:=\left\{i^{*}, \ldots, 4\right\}$ and $I_{0}:=\{\mathrm{m}, \mathrm{p}\} \cup\{1,2,3,4\} \backslash I_{1}$.

Set $\pi_{0}:=\lambda_{5}\left(\right.$ so $\left.\pi_{1}=\omega_{1} \cdot \lambda_{5}\right)$, and $\pi:=\pi_{1}+\lambda_{5}+\lambda_{5}$. Partition the final $\lambda_{5}$-interval of $\pi$, i.e. $\pi \backslash\left(\pi_{1}+\lambda_{5}\right)$, into sets $\Pi_{i}\left(i \in I_{0}\right)$ and $\Sigma_{i}\left(i \in I_{1}\right)$, each of size $\lambda_{5}$.

We construct a tidy $\mathrm{S} \lambda$-s iteration, using

$$
\Sigma:=\left\{\pi_{1}+\alpha: \alpha<\lambda_{5}\right\} \cup \bigcup_{i \in I_{1}} \Sigma_{i} \text { and } \Pi:=\bigcup_{i \in I_{0}} \Pi_{i} .
$$

We will satisfy the requirements of Corollary 5.17, so in particular inductively we will have $\left|\mathbb{P}_{\xi}^{*}\right|=\lambda_{5}$ (and so $\mathbb{P}_{\xi} \Vdash \mathfrak{c}=\lambda_{5}$ ) for all $\xi$.

(I1) At stage $\zeta \in\left[\pi_{1}, \pi_{1}+\lambda_{5}\right.$ ) (in particular, $\zeta \in \Sigma$ ), we just add Cohen reals. More formally, to fit our framework, we set $\mathbb{S}_{\zeta}=\mathbb{C}=\omega^{<\omega}$ (Cohen

16 Note that $\lambda_{\mathfrak{m}}=\aleph_{1}$ is allowed. 
forcing). Let $C_{\zeta}:=\emptyset$, which is closed and satisfies that $\mathbb{P}_{\zeta}^{-}:=\mathbb{P}_{\zeta}^{*}\left\lceil C_{\zeta}\right.$ (i.e., the set containing only the empty condition) is a complete subforcing of $\mathbb{P}_{\zeta}^{*}$. And $\mathbb{S}_{\zeta}^{V_{\zeta}^{\mathbb{P}_{\zeta}^{-}}}$is Cohen forcing in the ground model, which is Cohen forcing in any extension by absoluteness.

(I2) Assume $\zeta \in \Pi_{i}$ (for some $i \in I_{0}$ ).

(i) When $i=\mathrm{m}$, let $\Xi_{\zeta}$ be the family of all nice $\mathbb{P}_{\zeta}^{*}$-names of real-numberposets of size $<\lambda_{\mathfrak{m}}$ that are forced (by $\mathbb{P}_{\zeta}^{*}$ ) to be $k_{0}$-Knaster.

(ii) When $i=\mathrm{p}$, let $\Xi_{\zeta}$ be the family of all nice $\mathbb{P}_{\zeta}^{*}$-names of real-posets of size $<\lambda$ that are forced to be $\sigma$-centered.

(iii) When $i \in\{1,2,3\} \cap I_{0}$, we consider $\Xi_{\xi}$ as the family of nice $\mathbb{P}_{\zeta^{-}}$ names of all smaller-than- $\lambda_{i}$ versions of $\mathbb{S}_{i}$ in the $\mathbb{P}_{\zeta}^{*}$-extension, i.e., the forcings of the form

$$
Q=\mathbb{S}_{i} \cap E \text { where } E \text { is } \lambda_{i} \text {-elementary }
$$

as in Definition 6.3. Note that $\mathbb{S}_{i}$, and therefore also every variant $\mathbb{S}_{i} \cap E$, is linked and therefore Knaster.

(I3) If $\zeta \in \Sigma_{i}$ (for some $i \in I_{1}$, so $\lambda_{i}>\lambda$ ), we pick (by suitable bookkeeping) a $C_{\zeta} \subseteq \zeta^{+}$as in Lemma 5.15(b), i.e., $\left|C_{\zeta}\right|<\lambda_{i}, \mathbb{P}_{\zeta}^{-}:=\mathbb{P}^{*} \mid C_{\zeta} \lessdot \mathbb{P}_{\zeta}^{*}$, and we set $\mathbb{S}_{\zeta}:=\mathbb{S}_{i}^{V^{\mathbb{P}} \bar{\zeta}}$. (Here, suitable bookkeeping just means: For every $K \in\left[\pi^{+}\right]^{<\lambda_{i}}$ there is some index $\zeta$ such that $C_{\zeta} \supseteq K$.)

We can now show that the construction does what we want, apart from keeping $\mathfrak{b}$ small.

First let us note that sometimes it is more convenient to view $\mathbb{P}$ as a FS ccc iteration, where we first add the $\lambda_{5}$-many $\mathbb{G}_{\mathbf{B}}$ forcings (of size $\aleph_{1}$ ), then the $\lambda_{5}$-many Cohen reals, and then the rest of the iteration, where we interpret each FS product $\mathbb{Q}_{\zeta}$ for $\zeta \in \Pi_{i}$ as a FS iteration with index set $\lambda_{5}=\left|\Xi_{i}\right|$. So all in all we can represent $\mathbb{P}$ as a FS iteration

$$
\left\langle P_{\alpha}^{\prime}, \dot{Q}_{\alpha}^{\prime}\right\rangle_{\alpha \in \delta^{\prime}} \text { of length } \delta^{\prime}=\lambda_{5}+\lambda_{5}+\Sigma_{\zeta \in \pi \backslash\left(\lambda_{5}+\lambda_{5}\right)} \delta_{\zeta}^{\prime},
$$

$$
\text { with } \delta_{\zeta}^{\prime}:= \begin{cases}\lambda_{5} & \text { if } \zeta \in \Pi_{i}, \\ 1 & \text { otherwise. }\end{cases}
$$

For each $\alpha<\lambda_{5}+\lambda_{5},\left|\dot{Q}_{\alpha}^{\prime}\right| \leq \aleph_{1}$, and for each $\alpha \geq \lambda_{5}+\lambda_{5}$ in $\delta^{\prime}$, we say that $\dot{Q}_{\alpha}^{\prime}$ "is of type $i$ " for $i \in\{\mathrm{m}, \mathrm{p}, 1,2,3,4\}$, if either $\dot{Q}_{\alpha}^{\prime}=\dot{\mathbb{Q}}_{\zeta}$ for the respective $\zeta \in \Sigma_{i}$, or if $\dot{Q}_{\alpha}^{\prime}$ is a factor $\dot{Q}$ of $\dot{\mathbb{Q}}_{\zeta}$ for the respective $\zeta \in \Pi_{i}$. Note that $P_{\lambda_{5}}^{\prime}=\mathbb{P}_{\pi_{1}}$. 
LEmma 6.8: The construction above satisfies Goal 6.6, apart from possibly $\mathrm{LCU}_{3}$.

Proof. $\mathfrak{c}=\lambda_{5}$ : as we use a construction following Corollary 5.17.

Item (a) for $i=\mathrm{sp}$, i.e., $\mathrm{LCU}_{\mathrm{sp}}$ : This also follows from Corollary 5.17, and implies $\vdash_{\mathbb{P}} \mathfrak{s} \leq \lambda$.

$\mathfrak{p}=\mathfrak{s}=\lambda$ : To see $\mathfrak{p} \geq \lambda$ it is enough to show (in fact, equivalent, by Bell's theorem [Bel81]): For every $\sigma$-centered poset $Q^{\prime}$ of size $<\lambda$ (and contained in $\left.\omega^{\omega}\right)$, and any collection $D$ of size $<\lambda$ of dense subsets of $Q^{\prime}$, there is a $Q^{\prime}$-generic set over $D$. Any such $Q^{\prime}$ and $D$ are forced to be already in the $\mathbb{P}_{\alpha}$-extension for some $\alpha<\pi$. Pick some $\zeta \in \Pi_{\mathrm{p}}$ larger than $\alpha$. Then a name $\dot{Q}$ for $Q^{\prime}$ is used as factor of $\mathbb{P}_{\zeta}$, i.e., in $\mathbb{P}_{\zeta+1}$ there is a $\dot{Q}$-generic object (over $D$ ).

ZFC shows $\mathfrak{p} \leq \mathfrak{s}$, and as $\mathfrak{s} \leq \lambda$ we get equality.

Item (a) for $i \in\{1,2,4\}$, i.e., $\mathrm{LCU}_{i}$ : This is exactly as in [GKS19, §1.2]. For this argument we interpret $\mathbb{P}$ as the iteration $\left\langle P_{\alpha}^{\prime}, \dot{Q}_{\alpha}^{\prime}\right\rangle_{\alpha \in \delta^{\prime}}$ of (6.7). However, we work in the $\mathbb{P}_{\pi_{1}}$-extension (i.e., the $P_{\lambda_{5}}^{\prime}$-extension). So we investigate the forcing which first adds $\lambda_{5}$ many Cohens, and then a FS iteration of the iterands $Q_{\alpha}^{\prime}$.

As in [GKS19, §1.2], we now argue that each such $Q_{\alpha}^{\prime}$ is $\left(\mathbf{R}_{i}^{\mathrm{LCU}}, \lambda_{i}\right)$-good. ${ }^{17}$ So let us quickly check the cases (they are all summarized in [GKS19, Lemma 1.6], and use results from [JS90], [Kam89], [Bre91]). To get $\left(\mathbf{R}_{1}^{\mathrm{LCU}}, \lambda_{1}\right)$-good:

- If $Q_{\alpha}^{\prime}$ is of type m or type 1 , then $Q_{\alpha}^{\prime}$ has size $<\lambda_{1}$ and thus is $\left(\mathbf{R}_{i}^{\mathrm{LCU}}, \lambda_{1}\right)$ good (for any $i \in\{1,2,3,4\}$ ).

- If $Q_{\alpha}^{\prime}$ is of type p, 3 or 4 , then $Q_{\alpha}^{\prime}$ is $\sigma$-centered, and therefore $\left(\mathbf{R}_{1}^{\mathrm{LCU}}, \aleph_{1}\right)$ good.

- If $Q_{\alpha}^{\prime}$ is of type 2 , then it is a subalgebra of the measure algebra, and thus $\left(\mathbf{R}_{1}^{\mathrm{LCU}}, \aleph_{1}\right)$-good.

For $\left(\mathbf{R}_{2}^{\mathrm{LCU}}, \lambda_{2}\right)$-good the argument is even simpler: All $Q_{\alpha}^{\prime}$ have size $<\lambda_{2}$ or are $\sigma$-centered; and for $\left(\mathbf{R}_{4}^{\mathrm{LCU}}, \lambda_{4}\right)$-good the argument is trivial, as all $Q_{\alpha}^{\prime}$ have size $<\lambda_{4}$.

So this argument shows that, in the intermediate model $V^{\mathbb{P}_{\pi_{1}}}$, the rest $P^{\prime}$ of the forcing satisfies $\operatorname{LCU}_{i}\left(P^{\prime}, \lambda_{i}\right)$, witnessed by the Cohen reals

$$
\left\{\eta_{\alpha}: \alpha \in\left[\pi_{1}, \pi_{1}+\lambda_{i}\right)\right\}
$$

17 The notion $(R, \theta)$-good was introduced by Judah-Shelah [JS90] and Brendle [Bre91], definitions can also be found in [GKS19, Def. 1.5], [GMS16, Def. 3.2] or [BJ95, Def. 6.4.4]. 
This implies by definition of LCU that in the ground model $\mathrm{LCU}_{i}\left(\mathbb{P}, \lambda_{i}\right)$ holds, witnessed by the same Cohen reals.

Item (b) for $i \in I_{1}$, i.e., $\mathrm{COB}_{i}$ : This is also basically the same as in [GKS19, $\S 1.2]$, where this time we argue from the ground model $V$, not the intermediate model $V^{\mathbb{P}_{\pi_{1}}}$. We define the partial order $S_{i}$ to have domain $\Sigma_{i}$, ordered by $\zeta_{1} \leq_{S_{i}} \zeta_{2}$ iff $C_{\zeta_{1}} \subseteq C_{\zeta_{2}}$.

Note that $C_{\zeta}$ is in $\left[\pi^{+}\right]^{<\lambda_{i}},\left|\pi^{+}\right|=\lambda_{5}$, and our bookkeeping ensures that $S_{i}$ is $<\lambda_{i}$-directed. Corollary 5.10(c) together with the fact that $\lambda \leq \lambda_{i}$ shows that our bookkeeping will catch every real in the $\mathbb{P}$-extension. Therefore $S_{i}$, and the generics added at stages in $S_{i}$, witness the COB property.

Item (b) for $i \in I_{0} \cap\{1,2,3\}$ : This is very similar: Let $S_{i}$ be the set of pairs $(\zeta, \dot{E})$ such that $\zeta \in \Pi_{i}$ and $\dot{E}$ is a nice $\mathbb{P}_{\zeta}^{*}$-name of a $\lambda_{i}$-elementary subset of $\omega^{\omega}$. We order $S_{i}$ as follows: $\left(\xi_{1}, \dot{E}_{1}\right) \leq_{i}\left(\xi_{2}, \dot{E}_{2}\right)$ iff $\xi_{1} \leq \xi_{2}$ and the empty condition forces that $\dot{E}_{1} \subseteq \dot{E}_{2}$.

For $(\zeta, \dot{E}) \in S_{i}, \mathbb{S}_{i} \cap \dot{E}$ forms part of the FS product $\dot{\mathbb{Q}}_{\zeta}$, so $\mathbb{P}_{\zeta+1}$ adds a $\mathbb{S}_{i} \cap \dot{E}$ generic object $\dot{y}_{\zeta, \dot{E}}$ as in Fact 6.4 . We show that $S_{i}$ and $\left\{\dot{y}_{\zeta, \dot{E}}:(\zeta, \dot{E}) \in S_{i}\right\}$ witness $\mathrm{COB}_{i}$.

Let $\dot{r}$ be a $\mathbb{P}^{*}$-name of a real, then $\dot{r}$ is a $\mathbb{P}_{\xi_{0}}^{*}$-name of a real for some $\xi_{0}<\pi$, and there is some $\dot{E}_{0}$ such that $\left(\xi_{0}, \dot{E}_{0}\right) \in S_{i}$ and $\Vdash_{\mathbb{P}_{\xi_{0}}} \dot{r} \in \dot{E}_{0}$. Hence, whenever $(\xi, \dot{E}) \in S_{i}$ is above $\left(\xi_{0}, \dot{E}_{0}\right), \Vdash_{\mathbb{P}} \dot{r} \in \dot{E}$ so $\dot{y}_{\xi, \dot{E}}$ is generic over $\dot{r}$.

And for any $\left\langle\lambda_{i}\right.$-sequence $\left\langle E_{j}: j \in J\right\rangle$ of nice names for $\lambda_{i}$-elementary sets $E_{j}$ we can find a nice name for a $\lambda_{i}$-elementary set $E \supseteq \bigcup_{j \in J} E_{j}$. This shows that $S_{i}$ is $<\lambda_{i}$-directed.

Item (b) for $\boldsymbol{i}=\mathbf{s p}$, i.e., $\mathrm{COB}_{\mathrm{sp}}$ : This is basically the same: Among the $\sigma$ centered forcings that we use as factors in step $\zeta$ of type p, there are MathiasPrikry forcings $\mathbb{M}_{\dot{F}}$ on (free) filter bases of size $<\lambda$. In more detail: Assume $\dot{F}$ is a $\mathbb{P}_{\zeta}^{*}$-name for a filter base of size $\left\langle\lambda\right.$, so set $\dot{Q}:=\mathbb{M}_{\dot{F}}$. Then $\dot{Q}$ is $\sigma$-centered and adds a real which is not split by any set in $\dot{F}$.

So let $S_{\mathrm{sp}}$ be the set of pairs $(\zeta, \dot{F})$ such that $\zeta \in \Pi_{\mathrm{p}}$ and $\dot{F}$ is a nice $\mathbb{P}_{\zeta^{-}}^{*}$ name of a filter base of size $<\lambda$. Set $\left(\xi_{1}, \dot{F}_{1}\right) \leq_{\mathrm{p}}\left(\xi_{2}, \dot{F}_{2}\right)$ iff $\xi_{1} \leq \xi_{2}$ and the empty condition forces that $\dot{F}_{1} \subseteq \dot{F}_{2} \cup \dot{F}_{2}^{d}$, where $F^{d}:=\{\omega \backslash x: x \in F\}$. For $(\xi, \dot{F}) \in S_{\mathrm{p}}$, let $\dot{y}_{\xi, \dot{F}}$ be the $\mathbb{P}_{\xi+1}$-name of the generic real added by $\mathbb{M}_{\dot{F}}$. It follows that $S_{\mathrm{p}}$ and $\left\{\dot{y}_{\xi, \dot{F}}:(\xi, \dot{F}) \in S_{\mathrm{p}}\right\}$ witness $\mathrm{COB}_{\mathrm{sp}}$.

Item (d) is exactly the same as in [GKMSb, Lemma 4.7]. 
To guarantee $\mathfrak{b} \leq \lambda_{3}$, we have to make sure that the large iterands (i.e., the forcings of size $\geq \lambda_{3}$ ) do not destroy $\mathrm{LCU}_{3}$ (small forcings are, as usual, harmless). In our construction, the only large forcings are the partial eventually different forcings at steps $\zeta \in \Sigma_{4}$. For these forcings, we introduce in [GKS19] (based on [GMS16]) ultrafilter-limits and use them to preserve $\mathrm{LCU}_{3}$. The same argument works here.

Remark 6.9: Note that in the proof of Lemma 6.8 we do not require the hypotheses $\chi=\chi^{<\chi}$ and $\lambda_{3}=\chi^{+}$from Assumption 6.5. These will be used to guarantee $\mathrm{LCU}_{3}$ in the following subsection.

If in Assumption 6.5 we consider $\lambda_{3}=\lambda_{4}$ (instead of $\lambda_{3}<\lambda_{4}$ ), then the same proof of Lemma 6.8 guarantees Goal 6.6 in full (i.e., including LCU $_{3}$ ). When $\lambda_{\mathrm{sp}}=\lambda_{4}$, in the forcing construction above we have

$$
\Sigma=\left[\pi_{1}, \pi_{1}+\lambda_{5}\right) .
$$

\section{C. Dealing with $\mathfrak{b}$.}

Lemma 6.10: In addition to Assumption 6.5 we suppose that $2^{\chi} \geq \lambda_{5}$. Then we can choose $C_{\zeta}$ for all $\zeta \in \Sigma_{4}$ such that $\operatorname{LCU}_{3}(\mathbb{P}, \kappa)$ holds for all regular $\kappa \in\left[\lambda_{3}, \lambda_{5}\right]$. Moreover, in the inductive construction, for each $\zeta \in \Sigma_{4}$ there is a $\lambda$-club of $\left[\zeta^{+}\right]^{<\lambda_{4}}$ such that we can choose $C_{\zeta}$ from this club set.

Proof. This is analogous to [GKS19, §1.3], in particular to Lemma/Construction 1.30. We will only remark on the required changes. Again we interpret $\mathbb{P}$ as in (6.7).

We work from the ground model, not in the intermediate $\mathbb{P}_{\pi_{1}}$-extension. Accordingly, we have to incorporate the initial segment of the iteration $\mathbb{P}_{\pi_{1}}=P_{\lambda_{5}}^{\prime}$ into the argument. This is no problem, as we just have to deal with another type of small forcing, the $\dot{Q}_{\alpha}^{\prime}$ for $\alpha<\lambda_{5}$, which all have size $\aleph_{1}$.

Of course, $E^{\prime}:=\mathbb{E}^{V^{\mathbb{P}_{\zeta}^{*} / C_{\zeta}}}$ is closed under conjunctions of conditions, i.e., satisfies the assumptions of [GKS19, Fact 1.25]. And instead of "ground model code sequences" we use "nice $\mathbb{P}_{\zeta}^{*}\left\lceil C_{\zeta}\right.$-names".

The crucial part of the old proof is [GKS19, Lemma 1.30(d)]. There, we use the notation $w_{\alpha} \subseteq \alpha$, and $Q_{\alpha}$ are those $\mathbb{E}$-conditions that can be calculated in a Borel way from the generics with indices in $w_{\alpha}$, i.e., $Q_{\alpha}=\mathbb{E} \cap V^{\mathbb{P}^{*}} \uparrow w_{\alpha}$; and we show that the set of "suitable" $w_{\alpha}$ is an $\omega_{1}$-club in $[\alpha]^{<\lambda_{4}}$, where "suitable" 
means: If we have a ground-model-sequence of (nice) $Q_{\alpha}$-names, then the $D_{\alpha}^{\varepsilon}$ limit (a well-defined condition in eventually different forcing) is also element of $Q_{\alpha}$ (for all $\left.\varepsilon \in \chi\right)$.

The same argument gives us the following for our new framework: We can perform the construction of Lemma 6.8 and, at all indices $\zeta$ of type 4 , the set of "suitable" $C_{\zeta} \in\left[\zeta^{+}\right]^{<\lambda_{4}}$ is a $\lambda$-club, where suitable now means the following (recall that we have $\dot{\mathbb{Q}}_{\zeta}=\mathbb{E}^{\mathbb{P}_{\zeta}^{*} \mid C_{\zeta}}$ ): For any sequence of nice $\mathbb{P}_{\zeta}^{*}\left\lceil C_{\zeta}\right.$-names for elements of $\mathbb{E},{ }^{18}$ the $D_{\alpha}^{\varepsilon}$-limit of this sequence is forced to be in $\dot{\mathbb{Q}}_{\zeta}$ as well.

Here, we only get a $\lambda$-club and not an $\omega_{1}$-club, as only for increasing unions of length $\lambda$ we have

$$
\bigcup_{i \in \lambda}\left(\mathbb{P}_{\zeta}^{*}\left\lceil C_{i}\right)=\mathbb{P}_{\zeta}^{*} \uparrow\left(\bigcup_{i \in \lambda} C_{i}\right)\right.
$$

Also, we now have to choose $C_{\zeta}$ not only in this $\lambda$-club, but in the intersection with the $\lambda$-club of Lemma 5.15(b) (so that we get a closed $C_{\zeta}$ such that $\mathbb{P}_{\zeta}^{*}\left\lceil C_{\zeta} \lessdot \mathbb{P}_{\zeta}^{*}\right.$ as required for our construction).

The same argument as in the old proof (Lemma 1.31 there) then shows: Whenever all $C_{\zeta}$ are chosen "suitably" (for all $\zeta$ of type 4 ), we get $\mathrm{LCU}_{3}$.

Theorem 6.11: Assumption 6.5 is enough to find a $\mathbb{P}$ as required for Goal 6.6.

Proof. Let $R$ be the poset of partial functions $r: \chi \times \lambda_{5} \rightarrow\{0,1\}$ with domain of size $<\chi$ (ordered by extension). As we assume $\chi^{<\chi}=\chi$, this poset is $\chi^{+}$-cc, and obviously $<\chi$-closed, so it does not change any cofinalities. As in the old proof, at each step $\zeta$ of type 4 in the inductive construction of $\mathbb{P}$, we can go into the $R$-extension of the ground model, use Lemma 6.10 to get a suitable $C_{\zeta}^{0}$ (above some initial set given by the usual bookkeeping), find in $V$ some $\tilde{C}_{\zeta}^{0}$ such that $C_{\zeta}^{0}$ is forced to be a subset. Now we iterate this $\lambda$ many times (not just $\omega_{1}$ as in the old proof), taking unions at limits, and use the fact that the "suitable" parameters $C_{\zeta}$ are closed under $\lambda$-unions (they form a $\lambda$-club in $\left[\zeta^{+}\right]^{<\lambda_{4}}$ ).

This way we get a sequence of parameters $C_{\zeta}$ in the ground model, such that if we define in the $R$-extension a forcing $\mathbb{P}^{\prime}$ using these parameters we get $\mathrm{LCU}_{3}\left(\mathbb{P}^{\prime}, \kappa\right)$; a simple absoluteness argument [GKS19, Lemma 1.33] then shows that these parameters will already define in $V$ a forcing $\mathbb{P}$ with $\mathrm{LCU}_{3}(\mathbb{P}, \kappa)$.

18 Note: as $\left|C_{\zeta}\right|<\lambda_{4}$, and $\lambda_{4}$ is $\aleph_{1}$-inaccessible, there are $<\lambda_{4}$ many such sequences; cf. Lemma 5.5 (and 5.9). 
Note: We do not interpret $\Xi_{\zeta}$ (for $\zeta \in \Pi$ ) in the $R$-extension, but use it with the same meaning it has in $V$. So $\mathbb{P}^{\prime}$ may not be symmetric in the $R$-extension, but this is not important here: We are only interested in $\mathrm{LCU}_{3}\left(\mathbb{P}^{\prime}, \kappa\right)$ in this argument, and we do not claim that $\mathbb{P}^{\prime}$ in the $R$-extension satisfies the other properties we have already shown for $\mathbb{P}$. And for $\mathrm{LCU}_{3}\left(\mathbb{P}^{\prime}, \kappa\right)$, any iterand that has size $<\lambda_{3}$ is unproblematic.

Remark 6.12: It is not necessary to restrict $\lambda_{3}$ to a successor cardinal in Assumption 6.5. To allow regular $\lambda_{3}$ in general, we forget about $\chi$ in Assumption 6.5 and just assume that $\lambda_{3}^{<\lambda_{3}}=\lambda_{3}>\aleph_{1}$. In this way, Lemma 6.10 is valid by assuming $2^{\lambda_{3}} \geq \lambda_{5}$ instead, and Theorem 6.11 is true when replacing $\chi$ by $\lambda_{3}$ in the proof (i.e., $R$ gets modified and it forces $2^{\lambda_{3}} \geq \lambda_{5}$ ). No further changes in the proofs (even in those from [GKS19]) are needed to justify this.

On the other hand, can we allow $\lambda_{3}=\aleph_{1}$ in Assumption 6.5? (So all cardinals except $\lambda_{4}$ and $\lambda_{5}$ are $\aleph_{1}$.) Although we can make the construction in this case, now the forcings $\mathbb{G}_{\mathbf{B}_{\delta}}$ have size $\lambda_{3}=\aleph_{1}$, so they could destroy $\operatorname{LCU}_{3}\left(\mathbb{P}, \aleph_{1}\right)$. An alternative to deal with this problem is to perform a similar iteration with $\pi_{0}=0$ (so $\pi_{1}=0$, that is, no initial FS product of $\mathbb{G}_{\mathbf{B}}$ is used) and guarantee $\operatorname{LCU}_{\mathbf{R}^{*}}(\mathbb{P}, \kappa)$ for any regular $\kappa \in\left[\aleph_{1}, \lambda_{5}\right]$ with the methods of this subsection (i.e. the methods from [GKS19, $\S 1.3 \& \S 1.4]$ ) adapted to $\mathbf{R}^{*}$, where $\mathbf{R}^{*}$ is the Blass-uniform relational system from [KW96] (see also [Mej13, Example 2.19]) such that

$$
\mathfrak{b}\left(\mathbf{R}^{*}\right)=\max \{\mathfrak{b}, \mathfrak{s}\} \quad \text { and } \quad \mathfrak{d}\left(\mathbf{R}^{*}\right)=\min \{\mathfrak{d}, \mathfrak{r}\}
$$

6.D. The other constellations for the Knaster numbers. So far we have assumed that $\lambda_{\mathfrak{m}}>\aleph_{1}$ and that $k_{0}<\omega$. We now remark on how to prove the other cases:

CASE $\lambda_{\mathfrak{m}}=\aleph_{1}$. We only change $I_{0}:=\{\mathrm{p}\} \cup\left\{i \in[1,4]: \lambda_{i} \leq \lambda\right\}$ (so (I2)(i) is excluded in the construction). Check details in [GKMSb, Lemma 4.7]. Note that here the value of $k_{0}$ is irrelevant.

CASE $k_{0}=\omega$ AND $\lambda_{\mathfrak{m}}>\aleph_{1}$. Force with $P_{\text {cal }, \lambda_{\mathfrak{m}}} * \mathbb{P}$ where $P_{\text {cal }, \lambda_{\mathfrak{m}}}$ is the precaliber $\aleph_{1}$ poset from [GKMSb, §5] and $\mathbb{P}$ is the forcing resulting from the construction above (in the $P_{\text {cal }, \lambda_{\mathfrak{m}}}$-extension). ${ }^{19}$

19 For $i=\mathrm{m}$, recall that " $\omega$-Knaster" abbreviates "precaliber $\aleph_{1}$ ". 
6.E. The alternative order of the Left side. The construction of $[$ KST19, §2] for the alternative order of the left side of Cichoń's diagram can also be adapted in the situation of the previous theorems. This is just interchanging the order of the values of $\mathfrak{b}$ and $\operatorname{cov}(\mathcal{N})$, that is, instead of forcing $\operatorname{cov}(\mathcal{N})=\lambda_{2} \leq \mathfrak{b}=\lambda_{3}$, we force

$$
\mathfrak{b}=\lambda_{3}<\operatorname{cov}(\mathcal{N})=\lambda_{2} .
$$

See also [Mej19b] for the weakening of the hypothesis GCH:

Theorem 6.13: Theorem 6.11 (and Goal 6.6) is still valid when, in Assumption 6.5, we replace $\lambda_{1} \leq \lambda_{2} \leq \lambda_{3}<\lambda_{4}$ by $\lambda_{1} \leq \lambda_{3}<\lambda_{2}<\lambda_{4}{ }^{20}$

Remark 6.12 also applies in this situation.

\section{15 values}

In this section, we review some tools from [GKMSa, GKMSb] and show how they are used to control the cardinal characteristics other than $\mathfrak{s}$. We describe the forcing constructions but we omit the details in the proofs, since these are exactly as in the cited references.

We use the notions of $\mathfrak{m}$-like cardinal characteristic and $\mathfrak{h}$-like characteristic from [GKMSb, §3]. We do not need to recall their definition, but we only need some of their properties and to know that the cardinals $\mathfrak{m}_{k}(1 \leq k \leq \omega)$ are $\mathfrak{m}$-like, $\mathfrak{h}$ and $\mathfrak{g}$ are $\mathfrak{h}$-like, and $\mathfrak{p}$ and $\mathfrak{t}$ are of both types.

Lemma 7.1 ([GKMSb, Cor. 3.5]): Let $\kappa$ be an uncountable regular cardinal, $\lambda$ a cardinal, $\mathfrak{x}$ a cardinal characteristic, and let $\mathbb{P}$ be a $\kappa$-cc poset that forces $\mathfrak{x}=\lambda$ (so $\lambda$ is a cardinal in the $\mathbb{P}$ extension). If $M \preceq \mathcal{H}_{\chi}$ (with $\chi$ a large enough regular cardinal) is $<\kappa$ closed and contains (as elements) $\mathbb{P}, \kappa, \lambda$ and the parameters of the definition of $\mathfrak{x}$, then $\mathbb{P} \cap M$ is a complete subposet of $\mathbb{P}$ and:

(i) If $\mathfrak{x}$ is $\mathfrak{m}$-like and $\lambda \geq \kappa$, then $\mathbb{P} \cap M \Vdash \mathfrak{x} \geq \kappa$.

(ii) If $\mathfrak{x}$ is $\mathfrak{m}$-like and $\lambda<\kappa$, then $\mathbb{P} \cap M \Vdash \mathfrak{x}=\lambda$.

(iii) If $\mathfrak{x}$ is $\mathfrak{h}$-like, then $\mathbb{P} \cap M \Vdash \mathfrak{x} \leq|\lambda \cap M|$.

20 As in $[\mathrm{KST} 19, \S 2]$, the relational system $\mathbf{R}_{2}^{\mathrm{LCU}}$ corresponding to this result is not the same as the one for Theorem 6.11. Although this is a relational system of the reals, it is not Blass-uniform. 
Lemma 7.2 ([GKMSb, Lemma 6.3]): Assume:

(1) $\kappa \leq \nu$ are uncountable regular cardinals, $\mathbb{P}$ is a $\kappa$-cc poset.

(2) $\mu=\mu^{<\kappa} \geq \nu$ and $\mathbb{P}$ forces $\mathfrak{c}>\mu$.

(3) For some relational systems of the reals $\mathbf{R}_{i}^{1}\left(i \in I_{1}\right)$ and some regular $\lambda_{i}^{1} \leq \mu: \mathbb{P}$ forces $\operatorname{LCU}_{\mathbf{R}_{i}^{1}}\left(\lambda_{i}^{1}\right)$

(4) For some relational systems of the reals $\mathbf{R}_{i}^{2}\left(i \in I_{2}\right)$, and some directed order $S_{i}^{2}$ with $\mathfrak{b}\left(S_{i}^{2}\right)=\lambda_{i}^{2} \leq \mu$ and $\left|S_{i}^{2}\right| \leq \vartheta_{i}^{2} \leq \mu$ : $\mathbb{P}$ forces $\operatorname{COB}_{\mathbf{R}_{i}^{2}}\left(S_{i}^{2}\right)$.

(5) For some $\mathfrak{m}$-like characteristics $\mathfrak{y}_{j}(j \in J)$ and $\lambda_{j}<\kappa: \Vdash_{\mathbb{P}} \mathfrak{y}_{j}=\lambda_{j}$.

(6) For some $\mathfrak{m}$-like characteristics $\mathfrak{y}_{k}^{\prime}(k \in K): \Vdash_{\mathbb{P}} \mathfrak{y}_{k}^{\prime} \geq \kappa$.

(7) $\left|I_{1} \cup I_{2} \cup J \cup K\right| \leq \mu$.

Then there is a complete subforcing $\mathbb{P}^{*}$ of $\mathbb{P}$ of size $\mu$ forcing:

(a) $\mathfrak{y}_{j}=\lambda_{j}, \mathfrak{y}_{k}^{\prime} \geq \kappa, \operatorname{LCU}_{R_{i}^{1}}\left(\lambda_{i}^{1}\right)$ and $\operatorname{COB}_{R_{i^{\prime}}^{2}}\left(\lambda_{i^{\prime}}^{2}, \vartheta_{i^{\prime}}^{2}\right)$ for all $i \in I_{1}, i^{\prime} \in I_{2}$, $j \in J$ and $k \in K$;

(b) $\mathfrak{c}=\mu$ and $\mathfrak{g} \leq \nu$.

We are now ready to prove the main result of this paper. We use Notation 6.1 and the following assumption for all the results in this section.

\section{Assumption 7.3:}

(1) $\mu_{\mathfrak{m}} \leq \mu_{\mathfrak{p}} \leq \mu_{0} \leq \mu_{1} \leq \mu_{2} \leq \cdots \leq \mu_{8}$ are uncountable regular.

(2) $\mu_{9} \geq \mu_{8}$ is a cardinal such that $\mu_{9}^{<\mu_{0}}=\mu_{9}$.

(3) $0 \leq i_{0} \leq 2, \mu_{\mathrm{sp}} \in\left[\mu_{i_{0}}, \mu_{i_{0}+1}\right]$ and $\mu_{\mathfrak{r}} \in\left[\mu_{8-i_{0}}, \mu_{9-i_{0}}\right]$ are regular.

(4) There are eleven regular cardinals $\theta_{0}>\cdots>\theta_{10}>\mu_{9}$ such that $\theta_{i}^{<\theta_{i}}=\theta_{i}$ for any $i<11, \theta_{i}$ is $\aleph_{1}$-inaccessible for $i \in\{1,3,5,7\}, \theta_{3}=\chi_{3}^{+}$and $\chi_{3}=\chi_{3}^{<\chi_{3}} \cdot{ }^{21}$

Note that, under GCH, assumption (4) is irrelevant, and $\mu_{9}^{<\mu_{0}}=\mu_{9}$ is equivalent to

$$
\operatorname{cof}\left(\mu_{9}\right) \geq \mu_{0} .
$$

The Main Theorem for Figure 3(A) is proved in two steps through the following two results.

21 We could further weaken the assumption depending on the value $i_{0}$. For example, in case $i_{0}=1, \theta_{i}$ is required $\aleph_{1}$-inaccessible only for $i \in\{1,3,5\}$. Also, it is enough that $\theta_{0}^{<\theta_{1}}=\theta_{0}$ (here, $\theta_{0}$ could be singular), and $\theta_{3}$ is not needed successor according to Remark 6.12. For more pedantic weakenings, see [GKMSa, Rem. 3.5]. 
Theorem 7.4: Under Assumption 7.3, for any $k_{0} \in[2, \omega]$ there is a ccc poset $\mathbb{P}^{1}$ such that, for any $i \in\{1,2,3,4, \mathrm{sp}\}$,

(a) $\operatorname{LCU}_{i}\left(\mathbb{P}^{1}, \theta\right)$ holds for $\theta \in\left\{\mu_{i}, \mu_{9-i}\right\}$, where $\mu_{\mathrm{sp}}:=\mu_{\mathfrak{s}}$ and $\mu_{9-\mathrm{sp}}:=\mu_{\mathfrak{r}}$.

(b) There is some directed $S_{i}$ with $\operatorname{cp}\left(S_{i}\right)=\mu_{i}$ and $\operatorname{cof}\left(S_{i}\right)=\mu_{9-i}$ such that $\mathrm{COB}_{i}\left(\mathbb{P}^{1}, S_{i}\right)$ holds.

(c) $\mathbb{P}^{1}$ forces $\mathfrak{p}=\mathfrak{g}=\mu_{0}$ and $\mathfrak{c}=\mu_{9}$.

(d) $\mathbb{P}^{1}$ forces $\mathfrak{m}_{k}=\aleph_{1}$ for any $k \in\left[1, k_{0}\right)$, and $\mathfrak{m}_{k}=\mu_{\mathfrak{m}}$ for any $k \in\left[k_{0}, \omega\right]$.

Proof. We deal with the case $i_{0}=1$, that is, $\mu_{1} \leq \mu_{\mathrm{sp}} \leq \mu_{2}$ (any other case is similar). We rewrite the sequence

$$
\begin{gathered}
\mu_{1} \leq \mu_{\mathrm{sp}} \leq \mu_{2} \leq \mu_{3} \leq \mu_{4} \leq \mu_{5} \leq \mu_{6} \leq \mu_{\mathfrak{r}} \leq \mu_{7} \leq \mu_{8} \leq \mu_{9} \quad \text { as } \\
\vartheta_{10} \leq \vartheta_{8} \leq \vartheta_{6} \leq \vartheta_{4} \leq \vartheta_{2} \leq \vartheta_{1} \leq \vartheta_{3} \leq \vartheta_{5} \leq \vartheta_{7} \leq \vartheta_{9} \leq \vartheta_{11},
\end{gathered}
$$

and let $\left\langle\theta_{j}: j<11\right\rangle$ be cardinals as in Assumption 7.3(4) ordered by

$$
\vartheta_{11}<\theta_{10}<\theta_{9}<\cdots<\theta_{0}
$$

as shown in Figure 6.

Let $\mathbb{P}^{0}$ be the ccc poset obtained by application of Theorem 6.11 to $\lambda_{\mathfrak{m}}=\mu_{\mathfrak{m}}$, $\lambda_{1}=\theta_{9}, \lambda_{\mathrm{sp}}=\theta_{7}, \lambda_{2}=\theta_{5}, \lambda_{3}=\theta_{3}, \lambda_{4}=\theta_{1}$ and $\lambda_{5}=\theta_{0}$. In particular, this forces the top diagram of Figure 6 and item (d). We show how to construct a complete subforcing of $\mathbb{P}^{0}$ that satisfies the statement of the theorem, in particular, it forces the bottom diagram of Figure 6.

For $1 \leq n \leq 10$ and $\alpha<\vartheta_{n}$ define $M_{n, \alpha}$ fulfilling:

- $M_{n, \alpha} \preceq \mathcal{H}_{\chi}$ (for a fixed large enough regular $\chi$ ) and it contains (as elements) the sequences of $\theta$ 's and $\vartheta^{\prime}$ 's, $\mathbb{P}^{0}$ and the directed sets associated with the COB properties forced by $\mathbb{P}^{0}$.

- The sequences $\left\langle M_{m, \xi}: \xi<\vartheta_{m}\right\rangle$ for $1 \leq m<n$ and $\left\langle M_{n, \xi}: \xi<\alpha\right\rangle$ belong to $M_{n, \alpha}$.

- $M_{n, \alpha}$ is $<\theta_{n}$ closed of size $\theta_{n}$.

Set

$$
M_{n}:=\bigcup_{\alpha<\vartheta_{n}} M_{n, \alpha} \text { and } M^{+}:=\bigcap_{n=1}^{10} M_{n} .
$$

Exactly as in the proof of [GKMSa, Thm. 3.1] one can show that $M^{+} \preceq \mathcal{H}_{\chi}$, $M^{+}$is $\left\langle\vartheta_{10}\right.$-closed, and $\mathbb{P}^{\prime}:=\mathbb{P}^{0} \cap M^{+}$is a ccc poset that forces (a), (b) and $\mathfrak{c}=\theta_{10}$. Even more, $\mathbb{P}^{\prime}$ forces $(\mathrm{d})$ and $\mathfrak{p} \geq \vartheta_{10}$ by Lemma 7.1.

The desired poset is a complete subposet $\mathbb{P}_{1}$ of $\mathbb{P}^{\prime}$ of size $\vartheta_{11}$ obtained by direct application of Lemma 7.2 (to $\kappa=\nu=\mu_{0}$ and $\mu=\vartheta_{11}$ ). 


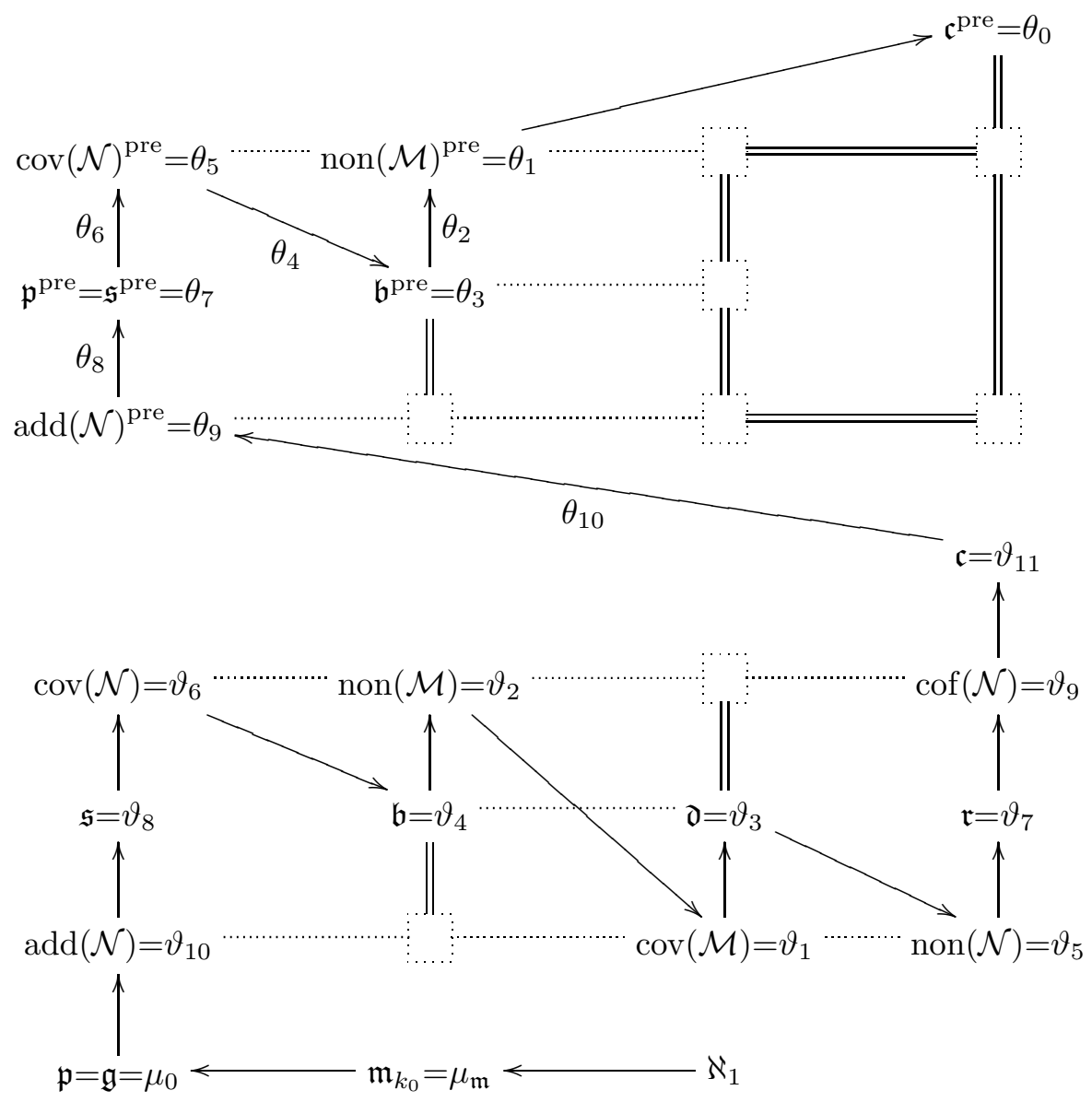

Figure 6 . The cardinals $\vartheta_{n}$ and $\theta_{n}$ are increasing along the arrows. The upper diagram shows the situation forced by $\mathbb{P}^{0}$, and the lower diagram shows the one forced by $\mathbb{P}^{1}$. ( $\mathfrak{s}$ can be anywhere between $\mathfrak{p}$ and $\mathfrak{b}$.)

TheOREm 7.5: Under Assumption 7.3, for any $k_{0} \in[2, \omega]$ there is a cofinality preserving poset $\mathbb{P}$ such that, for any $i \in\{1,2,3,4, \mathrm{sp}\}$, it satisfies (a), (b) and (d), and $\mathbb{P}$ forces $\mathfrak{p}=\mu_{\mathfrak{p}}, \mathfrak{h}=\mathfrak{g}=\mu_{0}$ and $\mathfrak{c}=\mu_{9}$. 
Proof. Let $\mathbb{Q}:=\mu_{\mathfrak{p}}^{<\mu_{\mathfrak{p}}}$ ordered by end extension, and let $\mathbb{P}^{1}$ be the poset constructed in Theorem 7.4. Exactly as in the proof of [GKMSb, Thm. 7.4], $\mathbb{P}:=\mathbb{P}^{1} \times \mathbb{Q}$ is as required.

In the same way, we can prove the Main Theorem corresponding to Figure $3(\mathrm{~B})$. In this case, the initial forcing $\mathbb{P}^{0}$ is obtained from Theorem 6.13.

Theorem 7.6: Both Theorems 7.4 and 7.5 are valid when Assumption 7.3 is modified in the following way:

(i) We replace the order of the regular cardinals in (1) by

$$
\mu_{\mathfrak{m}} \leq \mu_{\mathfrak{p}} \leq \mu_{0} \leq \mu_{1} \leq \mu_{3} \leq \mu_{2} \leq \mu_{4} \leq \mu_{5} \leq \mu_{7} \leq \mu_{6} \leq \mu_{8}
$$

(ii) In (3), we consider $i_{0} \in\{0,1\}$, but $\mu_{\mathrm{sp}} \in\left[\mu_{1}, \mu_{3}\right]$ and $\mu_{\mathfrak{r}} \in\left[\mu_{6}, \mu_{8}\right]$ when $i_{0}=1$.

(iii) In (4), instead of $\theta_{3}=\chi_{3}^{+}$and $\chi_{3}^{<\chi_{3}}=\chi_{3}$, assume $\theta_{5}=\chi_{5}^{+}$and $\chi_{5}^{<\chi_{5}}=\chi_{5}$.

\section{Discussions}

One obvious question is:

Question 1: How to separate additional cardinals from Figure 2?

Another one:

Question 2: How to get other orderings, where $\operatorname{non}(\mathcal{M})>\operatorname{cov}(\mathcal{M})$ ?

This is not possible with FS ccc iterations, as any such iteration whose length has uncountable cofinality $\delta$ forces $\operatorname{non}(\mathcal{M}) \leq \operatorname{cof}(\delta) \leq \operatorname{cov}(\mathcal{M})$, so alternative methods are required. A creature forcing method based on the notion of decisiveness [KS09, KS12] has been developed in [FGKS17] to separate five characteristics in Cichón's diagram, but this method is restricted to $\omega^{\omega}$-bounding forcings, i.e., results in $\mathfrak{d}=\omega_{1}$. An unbounded decisive creature construction might be promising. Alternatively, Brendle proposed a method of shattered iterations, ${ }^{22}$ which also may be a way to solve this problem.

Question 3: Are our main results (specifically, Theorems 6.11, 6.13, 7.4, 7.5 and 7.6) valid for $k_{0}=1$ ? I.e., can we force $\mathfrak{m}>\aleph_{1}$ ?

\footnotetext{
22 J. Brendle, personal communication.
} 
For $k_{0} \geq 2$ there was no problem to include, in our iterations, FS products of $k_{0}$-Knaster posets since they are still $k_{0}$-Knaster (hence ccc), but we cannot just use FS products of ccc posets because they do not produce ccc posets in general. In particular, we do not know how to modify Theorem 6.11 to force $\mathfrak{m}>\aleph_{1}$.

Question 4: Is it consistent with ZFC that $\mathfrak{b}<\mathfrak{s}<\operatorname{non}(\mathcal{M})<\operatorname{cov}(\mathcal{M})$ ?

In this paper $\mathfrak{s} \leq \mathfrak{b}$; and forcing $\mathfrak{s}>\mathfrak{b}$ is much more difficult, since MathiasPrikry posets may add dominating reals. Shelah [She84] proved the consistency of $\mathfrak{b}=\aleph_{1}<\mathfrak{s}=\mathfrak{c}=\aleph_{2}$ by a countable support iteration of proper posets. Much later, Brendle and Fischer [BF11] constructed an FS iteration via a matrix iteration to force $\aleph_{1}<\mathfrak{b}=\kappa<\mathfrak{s}=\mathfrak{c}=\lambda$ for arbitrarily chosen regular $\kappa<\lambda$. However, in this latter model, $\operatorname{non}(\mathcal{M})=\operatorname{cov}(\mathcal{M})=\mathfrak{c}$. It is not clear how to adapt Brendle's and Fischer's methods to our methods and produce a poset for the previous question.

\section{References}

[BCM21] J. Brendle, M. A. Cardona and D. A. Mejía, Filter-linkedness and its effect on preservation of cardinal characteristics, Annals of Pure and Applied Logic 171 (2021), Article no. 102856.

[BD85] J. E. Baumgartner and P. Dordal, Adjoining dominating functions, Journal of Symbolic Logic 50 (1985), 94-101.

[Bel81] M. G. Bell, On the combinatorial principle $P(\mathfrak{c})$, Fundamenta Mathematicae 114 (1981), 149-157.

[BF11] J. Brendle and V. Fischer, Mad families, splitting families and large continuum, Journal of Symbolic Logic 76 (2011), 198-208.

[BHHH04] B. Balcar, F. Hernández-Hernández and M. Hrušák, Combinatorics of dense subsets of the rationals, Fundamenta Mathematicae 183 (2004), 59-80.

[BJ95] T. Bartoszyński and H. Judah, Set Theory: On the Structure of the Real Line, A. K. Peters, Wellesley, MA, 1995.

[Bla10] A. Blass, Combinatorial cardinal characteristics of the continuum, in Handbook of Set Theory. Vols. 1, 2, 3, Springer, Dordrecht, 2010, pp. 395-489.

[Bre91] J. Brendle, Larger cardinals in Cichoń's diagram, Journal of Symbolic Logic 56 (1991), 795-810.

[DS18] A. Dow and S. Shelah, On the cofinality of the splitting number, Koninklijke Nederlandse Akademie van Wetenschappen. Indagationes Mathematicae 29 (2018), 382-395.

[EK65] R. Engelking and M. Karłowicz, Some theorems of set theory and their topological consequences, Fundamenta Mathematicae 57 (1965), 275-285.

[FFMM18] V. Fischer, S. D. Friedman, D. A. Mejía and D. C. Montoya, Coherent systems of finite support iterations, Journal of Symbolic Logic 83 (2018), 208-236. 
[FGKS17] A. Fischer, M. Goldstern, J. Kellner and S. Shelah, Creature forcing and five cardinal characteristics in Cichon's diagram, Archive for Mathematical Logic 56 (2017), 1045-1103.

[GKMSa] M. Goldstern, J. Kellner, D. A. Mejía and S. Shelah, Cichońs maximum without large cardinals, Journal of the European Mathematical Society, to appear, https://arxiv.org/abs/1906.06608.

[GKMSb] M. Goldstern, J. Kellner, D. A. Mejía and S. Shelah, Controlling classical cardinal characteristics without adding reals, Journal of Mathematical Logic, https://doi.org/10.1142/S0219061321500185.

[GKS19] M. Goldstern, J. Kellner and S. Shelah, Cichoń's maximum, Annals of Mathematics 190 (2019), 113-143.

[GMS16] M. Goldstern, D. A. Mejía and S. Shelah, The left side of Cichoń's diagram, Proceedings of the American Mathematical Society 144 (2016), 4025-4042.

[Hec72] S. H. Hechler, Short complete nested sequences in $\beta N \backslash N$ and small maximal almost-disjoint families, General Topology and its Applications 2 (1972), 139149.

[JS88] H. Judah and S. Shelah, Souslin forcing, Journal of Symbolic Logic 53 (1988), 1188-1207.

[JS90] H. Judah and S. Shelah, The Kunen-Miller chart (Lebesgue measure, the Baire property, Laver reals and preservation theorems for forcing, Journal of Symbolic Logic 55 (1990), 909-927.

[Kam89] A. Kamburelis, Iterations of Boolean algebras with measure, Archive for Mathematical Logic 29 (1989), 21-28.

[KS09] J. Kellner and S. Shelah, Decisive creatures and large continuum, Journal of Symbolic Logic 74 (2009), 73-104.

[KS12] J. Kellner and S. Shelah, Creature forcing and large continuum: the joy of halving, Archive for Mathematical Logic 51 (2012), 49-70.

[KST19] J. Kellner, S. Shelah and A. Tănasie, Another ordering of the ten cardinal characteristics in cichon's diagram, Commentationes Mathematicae Universitatis Carolinae 60 (2019), 61-95.

[Kun80] K. Kunen, Set Theory, Studies in Logic and the Foundations of Mathematics, Vol. 102, North-Holland, Amsterdam-New York, 1980.

[KW96] A. Kamburelis and B. Weglorz, Splittings, Archive for Mathematical Logic 35 (1996), 263-277.

[Mej13] D. A. Mejía, Models of some cardinal invariants with large continuum, Kyōto Daigaku Sūrikaiseki Kenkyūsho Kōkyūroku 1851 (2013), 36-48..

[Mej19a] D. A. Mejía, Matrix iterations with vertical support restrictions, Proceedings of the 14th and 15th Asian Logic Conferences, World Scientific, Hackensack, NJ, 2019, pp. 213-248.

[Mej19b] D. A. Mejía, A note on "Another ordering of the ten cardinal characteristics in Cichoń's Diagram" and further remarks, Kyōto Daigaku Sūrikaiseki Kenkyūsho Kōkyūroku 2141 (2019), 1-15. 
[MS16] M. Malliaris and S. Shelah, Cofinality spectrum theorems in model theory, set theory, and general topology, Journal of the American Mathematical Society 29 (2016), 237-297.

[She84] S. Shelah, On cardinal invariants of the continuum, in Axiomatic Set Theory (Boulder, Colo., 1983), Contemporary Mathematics, Vol. 31, American Mathematical Society, Providence, RI, 1984, pp. 183-207.

[She00] S. Shelah, Covering of the null ideal may have countable cofinality, Fundamenta Mathematicae 166 (2000), 109-136.

[Ste93] J. Steprāns, Combinatorial consequences of adding Cohen reals, in Set Theory of the Reals (Ramat Gan, 1991), Israel Mathematical Conference Proceedings, Vol. 6, Bar-Ilan University, Ramat Gan, 1993, pp. 583-617.

[Voj93] P. Vojtáš, Generalized Galois-Tukey-connections between explicit relations on classical objects of real analysis, in Set Theory of the Reals (Ramat Gan, 1991), Israel Mathematical Conference Proceedings, Vol. 6, Bar-Ilan University, Ramat Gan, 1993, pp. 619-643. 